\title{
Comparing human and model-based forecasts of COVID-19 in Germany and Poland
}

Nikos I. Bosse, Sam Abbott, Johannes Bracher, Habakuk Hain, Billy J. Quilty, Mark Jit, Centre for the Mathematical Modelling of Infectious Diseases COVID-19 Working Group, Edwin van Leeuwen, Anne Cori, Sebastian Funk

\section{Abstract}

Forecasts based on epidemiological modelling have played an important role in shaping public policy throughout the COVID-19 pandemic. This modelling combines knowledge about infectious disease dynamics with the subjective opinion of the researcher who develops and refines the model and often also adjusts model outputs. Developing a forecast model is difficult, resource- and time-consuming. It is therefore worth asking what modelling is able to add beyond the subjective opinion of the researcher alone. To investigate this, we analysed different real-time forecasts of cases of and deaths from COVID-19 in Germany and Poland over a 1-4 week horizon submitted to the German and Polish Forecast Hub. We compared crowd forecasts elicited from researchers and volunteers, against a) forecasts from two semi-mechanistic models based on common epidemiological assumptions and b) the ensemble of all other models submitted to the Forecast Hub. We found crowd forecasts, despite being overconfident, to outperform all other methods across all forecast horizons when forecasting cases (weighted interval score relative to the Hub ensemble 2 weeks ahead: 0.89). Forecasts based on computational models performed comparably better when predicting deaths (rel. WIS 1.26), suggesting that epidemiological modelling and human judgement can complement each other in important ways.

\section{Introduction}

Infectious disease modelling has a long tradition and has helped inform public health decisions both through scenario modelling, as well as actual forecasts of (among others) influenza (Biggerstaff et al., 2016; e.g. McGowan et al., 2019; Reich, Brooks, et al., 2019; Shaman \& Karspeck, 2012), dengue fever (Colón-González et al., 2021; e.g. Johansson et al., 2019; Yamana et al., 2016), ebola (Funk et al., 2019; e.g. Viboud et al., 2018), chikungunya (e.g. Del Valle et al., 2018; Farrow et al., 2017) and now COVID-19 (Bracher, Wolffram, Deuschel, Görgen, Ketterer, Ullrich, Abbott, Barbarossa, Bertsimas, Bhatia, Bodych, Bosse, Burgard, Castro, et al., 2021; Bracher, Wolffram, Deuschel, Görgen, Ketterer, Ullrich, Abbott, Barbarossa, Bertsimas, Bhatia, Bodych, Bosse, Burgard, Fiedler, et al., 2021; Cramer et al., 2021; Cramer et al., 2020; European Covid-19 Forecast Hub, 2021; e.g. Funk et al., 2020). Applications of epidemiological models differ in the way they make statements about the future. Forecasts aim to predict the future as it will occur, while scenario modelling and projections aim to represent what the future could look like under certain scenario assumptions or if conditions stayed the same as they were in the past. Forecasts can be judged by comparing them against observed data. Since it is much harder to fairly assess the accuracy and usefulness of projections and scenario modelling in the same way, this work focuses on forecasts, which represent only a subset of all epidemiological modelling.

Since March 2020, forecasts of COVID-19 from multiple teams have been collected, aggregated and compared by Forecast Hubs such as the US Forecast Hub (Cramer et al., 2021; Cramer et al., 2020), the German and Polish Forecast Hub (Bracher, Wolffram, Deuschel, Görgen, Ketterer, Ullrich, Abbott, Barbarossa, Bertsimas, Bhatia, Bodych, Bosse, Burgard, Castro, et al., 2021; Bracher, Wolffram, Deuschel, Görgen, Ketterer, Ullrich, Abbott, Barbarossa, Bertsimas, Bhatia, Bodych, Bosse, Burgard, Fiedler, et al., 2021) and the European Forecast Hub (European Covid-19 Forecast Hub, 2021). Often, different individual forecasts are combined 
into a single forecast, e.g. by taking the mean or median of all forecasts. These ensemble forecasts usually tend to perform better and more consistently than individual forecasts (see e.g. Yamana et al. (2016); Reich, McGowan, et al. (2019)).

Individual computational models usually rely to varying degrees on mechanistic assumptions about infectious disease dynamics (such as SIR-type compartmental models that aim to represent how individuals move from being susceptible to infected and then recovered or dead). Some are more statistical in nature (such as time series models that detect statistical patterns without explicitly modelling disease dynamics). How exactly such a mathematical or computational model is constructed and which assumptions are made depends on subjective opinion and judgement of the researcher who develops and refines the model. Models are commonly adjusted and improved based on whether the model output looks plausible to the researchers involved.

The process of model construction and refinement is laborious and time-consuming, and it is therefore worth asking what modelling can add beyond the subjective judgment of the researcher alone. In this work, we ask this question specifically in the context of predictive performance, and set aside other advantages of epidemiological modelling (such as reproducibility or the ability to obtain a deeper fundamental understanding of how diseases spread). One natural way to do this is to compare the predictive performance of forecasts based on computational models ("model-based forecasts") against forecasts made by individual humans without explicit use of a computer model ("direct human forecasts") or a combination of multiple such forecasts ("crowd forecasts").

Previous work has examined such direct human forecasts in various contexts, such as geopolitics (Atanasov et al., 2016; Tetlock et al., 2014), meta-science (Hoogeveen et al., 2020; ReplicationMarkets, 2020), sports (Servan-Schreiber et al., 2004) and epidemiology (Farrow et al., 2017; McAndrew \& Reich, 2020; Recchia et al., 2021). Several prediction platforms (CSET Foretell, 2021; Hypermind, 2021; Metaculus, 2020) and prediction markets (PredictIt, 2021) have been created to collate expert and non-expert predictions. However, with the notable exception of Farrow et al. (2017), these forecasts were not designed to be evaluated alongside model-based forecasts and usually follow their own (often binary) prediction formats. Direct human forecasts may be able to take into account insights and relationships between variables which are hard to specify using epidemiological models. However, it is not entirely clear in which situations human forecasts perform well or badly. For example, Farrow et al. (2017) found that humans could outperform computer models at predicting the 2014/15 and 2015/16 flu season in the US, a setting where the disease was well known and information about previous seasons was available. However, humans tended to do slightly worse at predicting the 2014/15 outbreak of chikungunya in the Americas, a disease previously largely unobserved and unknown in these regions at the time.

In this study, we analyse the performance of direct human forecasts relative to model-based forecasts and discuss the added benefit of epidemiological modelling over human judgement alone. As a case study, we use different forecasts, involving varying degrees of human intervention, which we submitted in real time to the German and Polish Forecast Hub. In contrast to Farrow et al. (2017) we elicited not only point predictions, but full predictive distributions ("probabilistic forecasts", see e.g. Held et al. (2017)) from participants. This allows us to compare not only predictive accuracy, but also how well human forecasters and model-based forecasts were able to quantify forecast uncertainty.

\section{Methods}

We created and submitted the following forecasts to the German and Polish Forecast Hub: 1) a direct human forecast (henceforth called "crowd forecast"), elicited from participants through a web application (Bosse, Abbott, EpiForecasts, et al., 2020b) and 2) two semi-mechanistic model-based forecasts ("renewal model" and "convolution model") informed by basic assumptions about COVID-19 epidemiology. While the two semi-mechanistic forecasts were necessarily shaped by our implicit assumptions and decisions, they were designed such as to minimise the amount of human intervention involved. For example, we refrained from adjusting model outputs or refining the models based on past performance. Forecasts were created in real time over a period of 21 weeks from October 12th 2020 until March 1st 2021 and submitted to the German and Polish Forecast hub (Bracher, Wolffram, Deuschel, Görgen, Ketterer, Ullrich, Abbott, 
Barbarossa, Bertsimas, Bhatia, Bodych, Bosse, Burgard, Castro, et al., 2021; Bracher, Wolffram, Deuschel, Görgen, Ketterer, Ullrich, Abbott, Barbarossa, Bertsimas, Bhatia, Bodych, Bosse, Burgard, Fiedler, et al., 2021). All code and tools necessary to generate the forecasts and make a forecast submission are available in the covid.german.forecasts R package (Bosse, Abbott, EpiForecasts, et al., 2020a). This repository also contains a record of all forecasts submitted to the German and Polish Forecast Hub. Forecasts were evaluated using a variety of scoring metrics and compared among each other and against an ensemble of all other models submitted to the German and Polish Forecast Hub.

\subsection{Forecast targets and interaction with the German and Polish Forecast Hub}

The German and Polish Forecast Hub (now mostly merged into the European Covid-19 Forecast Hub (2021)) elicits predictions for various COVID-19 related forecast targets from different research groups every week. Forecasts had to be made every Monday (with submissions allowed until Tuesday 3pm) and were permitted to use any data that was available by Monday 11.59pm. We submitted forecasts for incident and cumulative weekly reported numbers of cases of and deaths from COVID-19 on a national level in Germany and Poland over a one to four week forecast horizon. Forecasts were submitted on Mondays, but weeks were defined as ending on a Saturday (and starting on Sunday), meaning that forecast horizons were in fact 5, 12, 19 and 26 days. Submissions were required in a quantile-based format with 23 quantiles of each output measure at levels $0.01,0.025,0.05,0.10,0.15, \ldots, 0.95,0.975,0.99$. Forecasts submitted to the Forecast Hub were combined into different ensembles every week, with the median ensemble (i.e., the $\alpha$-quantile of the ensemble is given by the median of all submitted $\alpha$-quantiles) being the default ensemble shown on all official Forecast hub visualisations (https://kitmetricslab.github.io/forecasthub/forecast).

Data on daily reported test positive cases and deaths linked to COVID-19 were provided by the organisers of the German and Polish Forecast hub. Until December 14th, 2020, these data were sourced from the European Centre for Disease Control (ECDC, 2020). After ECDC stopped publishing daily data, observations were sourced from the Robert Koch Institute (RKI) and the Polish Ministry of Health for the remainder of the submission period (RKI, 2021). These data are subject to reporting artefacts, (such as for example delayed case reporting in Poland on the 24th November, Forsal.pl (2020)), changes in reporting over time, and variation in testing regimes (for example in Germany from the 11th of November on, Ärzteblatt (2020)).

\subsection{Crowd forecasts}

Our crowd forecasts were created as an ensemble of forecasts made by individual participants every week through a web application (https://cmmid-lshtm.shinyapps.io/crowd-forecast/). Weekly forecasts had to be submitted before Tuesday $12 \mathrm{pm}$ every week, but participants were asked to only use any information or data that was already available by Monday night. The application was built using the shiny and golem R packages (Chang et al., 2021; Fay et al., 2021) and is available in the crowdforecastr R package (Bosse, Abbott, EpiForecasts, et al., 2020b). To make a forecast in the application participants could select a predictive distribution (with the default being log-normal) to represent the probability that the forecasted quantity took certain values. Median and width of the uncertainty could be adjusted by either interacting with a figure showing their forecast or providing numerical values (see screenshot in Figure 5 in the SI). The default shown was a repetition of the last known observation with constant uncertainty around it computed as the standard deviation of the last four changes in weekly log observed forecasts (i.e. as $\sigma(\log ($ value 4$)-\log ($ value 3$), \log ($ value 3$)-\log ($ value 2$), \ldots))$. Our interface also allowed participants to view past observations based on the hub data, as well as their forecasts, on a logarithmic scale and presented additional contextual COVID-19 data sourced from Our World in Data (2020). These data included, for example, notifications of both test positive COVID-19 cases and COVID-19 linked deaths and the number of COVID-19 tests conducted over time.

Forecasts were stored in a Google Sheet and downloaded, cleaned and processed every week for submission to the Forecast Hub. If a forecaster had submitted multiple predictions for a single target, only the latest submission was kept. Information on the chosen distribution as well as the parameters for median and width were used to obtain the required set of 23 quantiles from that distribution. Forecasts from all forecasters were then aggregated using an unweighted quantile-wise mean (i.e., the $\alpha$-quantile of the ensemble is given 
by the mean of all submitted $\alpha$-quantiles). On a few occasions, individual forecasts were assessed as clearly erroneous by visual inspection and subsequently removed before aggregation and were excluded from the submission as well as the analysis.

Participants were recruited mostly within the Centre of Mathematical Modeling of Infectious Diseases at the London School of Hygiene \& Tropical Medicine, but participants were also invited personally or via social media to submit predictions. Depending on whether they had a background in either statistics, forecasting or epidemiology, participants were asked to self-identify as 'experts' or 'non-experts'.

\subsection{Model-based forecasts}

We used two Bayesian semi-mechanistic models from the EpiNow2 R package (version 1.3.3) as our modelbased forecasts (Abbott, Hellewell, Hickson, et al., 2020). The first of these models, here called "renewal model", used the renewal equation (Fraser, 2007) to predict reported cases and deaths (see details in the SI). It estimated the effective reproduction number $R_{t}$ (the average number of people each person infected at time $\mathrm{t}$ is expected to infect in turn) and modelled future infections as a weighted sum of past infection multiplied by $R_{t}$. $R_{t}$ was assumed to stay constant beyond the forecast date, roughly corresponding to continuing the latest exponential trend in infections. On the 9 th of November we altered the date when $R_{t}$ was assumed to be constant from two weeks prior to the date of the forecast to the forecast date, which we found to yield a more stable $R_{t}$ estimate. Reported case and death notifications were obtained by convolving predicted infections over data-based delay distributions (Abbott, Hellewell, Thompson, et al., 2020; Abbott, Hellewell, Hickson, et al., 2020; epiforecasts.io/covid, 2020; Sherratt et al., 2021) to model the time between infection and report date. The renewal model was used to predict cases as well as deaths with forecasts being generated for each target separately. Death forecasts from the renewal model were therefore not informed by past cases. One submission of the renewal model on December 28th 2020 was delayed and therefore not included in the official Forecast hub ensemble.

The second model ("convolution model", see details in SI) was only used to forecast deaths and was added later, starting December 7th 2020 (with the first forecast from December 7th suffering from a software bug and therefore disregarded in all further analyses). The convolution model was submitted, but never included in the official Forecast hub ensemble due to concerns that it could be too similar to the renewal model. The convolution model predicted deaths as a fraction of infected people who would die with some delay, by using a convolution of reported cases with a distribution that described the delay from case report to death and a scaling factor (the case-fatality ratio). Both the renewal and the convolution model used daily observations and assumed a negative binomial observation model with a multiplicative day-of-the-week effect (Abbott, Hellewell, Hickson, et al., 2020).

Line list data used to inform the prior for the delay from symptom onset to test positive case report or death in the model-based forecasts was sourced from (Xu et al., 2020) with data available up to the 1st of August. All model fitting was done using Markov-chain Monte Carlo (MCMC) in stan (Stan Development Team, 2020) with each location and forecast target being fitted separately.

\subsection{Analysis}

For the main analysis we focused mostly on two week ahead forecasts, as COVID-19 forecasts, especially for cases, were in the past found to have poor predictive performance beyond this horizon (Bracher, Wolffram, Deuschel, Görgen, Ketterer, Ullrich, Abbott, Barbarossa, Bertsimas, Bhatia, Bodych, Bosse, Burgard, Castro, et al., 2021). Forecasts for cases were scored using the full period from October 2020 until March 2021. To ensure comparability between models, all death forecasts were scored using only the period from December 14th on, where all models including the convolution model were available. To ensure robustness of our results we conducted a sensitivity analysis where all forecasts (including cases) were scored only over the later period for which all forecasts were available (see Section A.11 in the SI). Results remained broadly unchanged.

Forecasts were analysed using the following scoring metrics: The weighted interval score (WIS) (Bracher, Ray, et al., 2021), the absolute error, relative bias, and empirical coverage of the $50 \%$ and $90 \%$ prediction intervals. The WIS is a proper scoring rule (Gneiting \& Raftery, 2007), meaning that in expectation the score 
is optimised by reporting a predictive distribution that is identical to the true data-generating distribution. Forecasters are therefore incentivised to report their true belief about the future. The WIS can be understood as a generalisation of the absolute error to quantile-based forecasts (also meaning that smaller values are better) and can be decomposed into three separate penalties: forecast spread (i.e. uncertainty of forecasts), over-prediction and under-prediction. While the over- and under-prediction components of the WIS capture the amount of over-prediction and under-prediction in absolute terms, we also look at a relative tendency to make biased forecasts. The bias metric (Funk et al., 2019) we use captures how much probability mass of the forecast was above or below the true value (mapped to values between -1 and 1 ) and therefore represents a general tendency to over- or under-predict in relative terms. A value of -1 implies that all quantiles of the predictive distribution are below the observed value and a value of 1 that all quantiles are above the observed value. Empirical coverage is the percentage of observed values that fall inside a given prediction interval (e.g. how many observed values fall inside all $50 \%$ prediction intervals). Scoring metrics are explained in more detail in Table 1 in the SI. All scores were calculated using the scoringutils R package (Bosse, Abbott, EpiForecasts, et al., 2020c).

At all stages of the evaluation our forecasts were compared to the median ensemble of all other models submitted to the German and Polish Forecast Hub ("Hub ensemble"). This "Hub ensemble" was retrospectively computed and excludes all our models. It therefore differs from the official Hub ensemble (here called "hub-ensemble-realised") which included crowd forecasts as well as renewal model forecasts. To enhance interpretability of scores we mainly report WIS relative to the Hub ensemble in the main text, i.e. we divided the average scores for a given model by the average score achieved by the Hub ensemble on the same set of forecasts (with values $>1$ implying worse and values $<1$ implying better performance than the Hub ensemble). In addition to comparing our forecasts against the hub ensemble excluding our models, we also assessed the impact of our forecasts on the performance of the forecasting hub by recalculating separate versions of the Hub ensemble with only some (or all) of our forecasts included. Versions that included either all of our models ("hub-ensemble-with-all") or only one of them ("hub-ensemble-with-X") were computed retrospectively.

\section{Results}

\subsection{Crowd forecast participation}

A total number of 32 participants submitted forecasts, 17 of those self-identified as 'expert' in either forecasting or epidemiology. The median number of forecasters for any given forecast target was 6 , the minimum 2 and the maximum 10. The mean number of submissions from an individual forecaster was 4.7 but the median number was only one - most participants dropped out after their first submission. Only two participants submitted a forecast every single week, both of whom are authors on this study. 
A

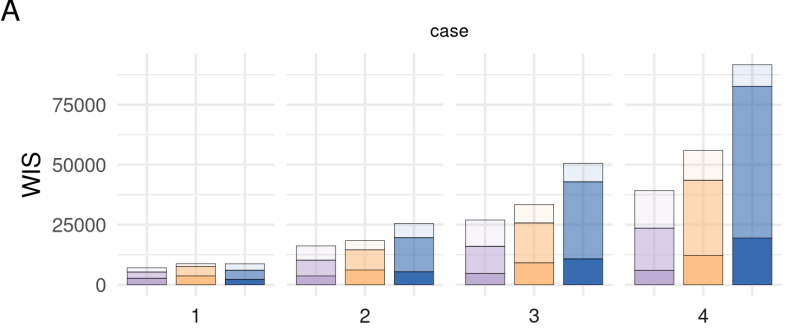

C

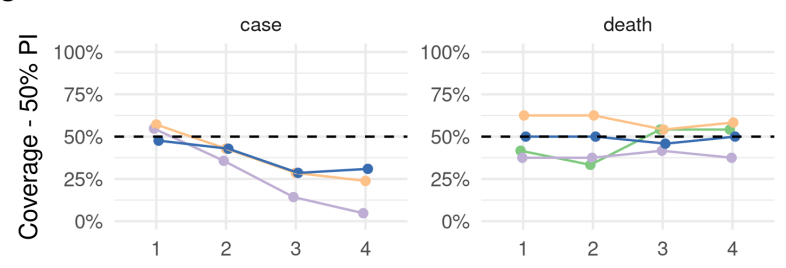

$E$

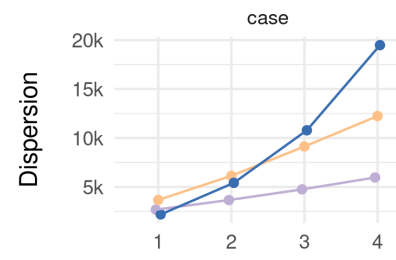

G
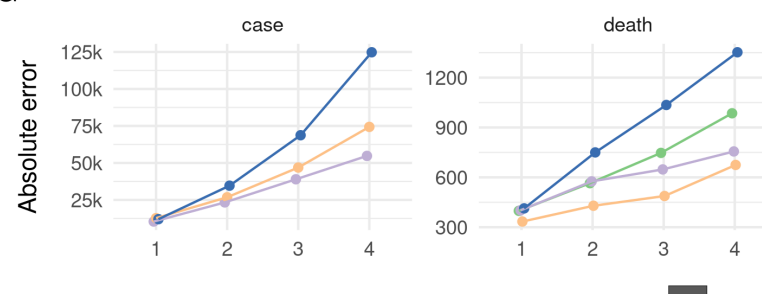

B

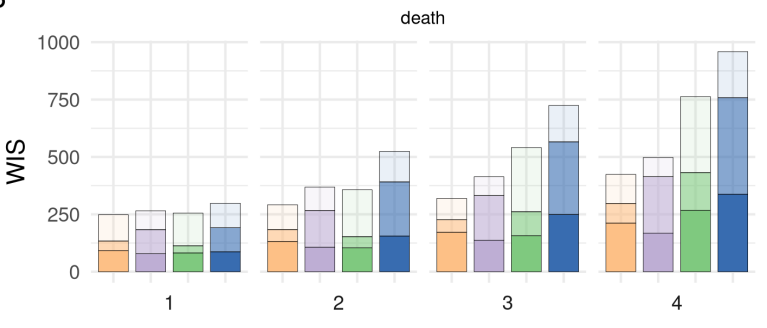

D

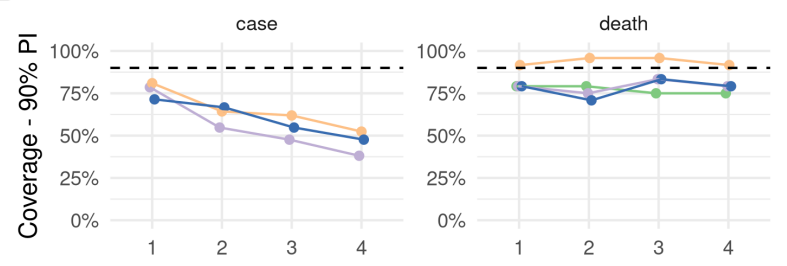

$\mathrm{F}$

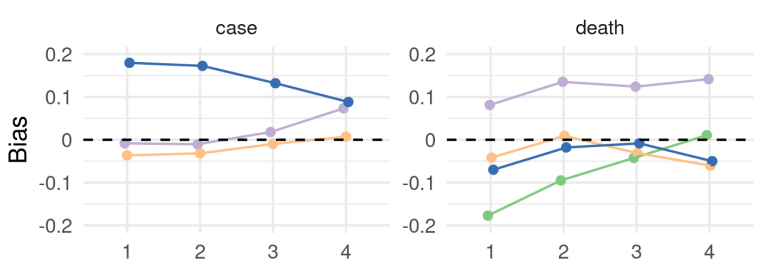

$\mathrm{H}$
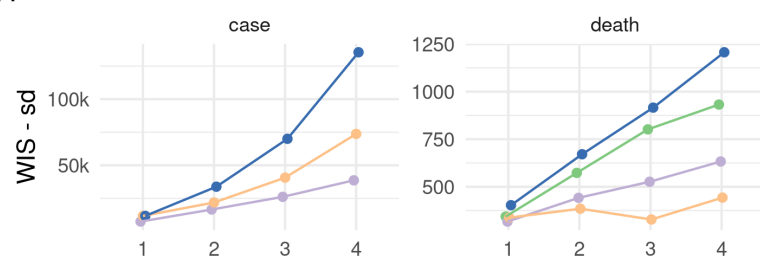

WIS components Dispersion

Under-prediction $\quad$ Over-prediction

Model $\square$ Convolution $\square$ Crowd forecast $\square$ Hub-ensemble $\square$ Renewal

Figure 1: Visualisation of aggregate performance metrics across for forecasts one to four weeks into the future (marked as 1 - 4 on the $\mathrm{x}$ axis). A, B: mean weighted interval score (WIS, lower indicates better performance) across horizons. WIS is decomposed into its components dispersion, over-prediction and under-prediction. C: Empirical coverage of the $50 \%$ prediction intervals (50\% coverage is perfect). D: Empirical coverage of the $90 \%$ prediction intervals. E: Dispersion (same as in panel A, B). Higher values mean greater dispersion of the forecast and imply ceteris paribus a worse score. F: Bias, i.e. general (relative) tendency to over- or underpredict. Values are between -1 (complete under-prediction) and 1 (complete over-prediction) and 0 ideally. G: Absolute error of the median forecast (lower is better). H. Standard deviation of all WIS values for different horizons 
A

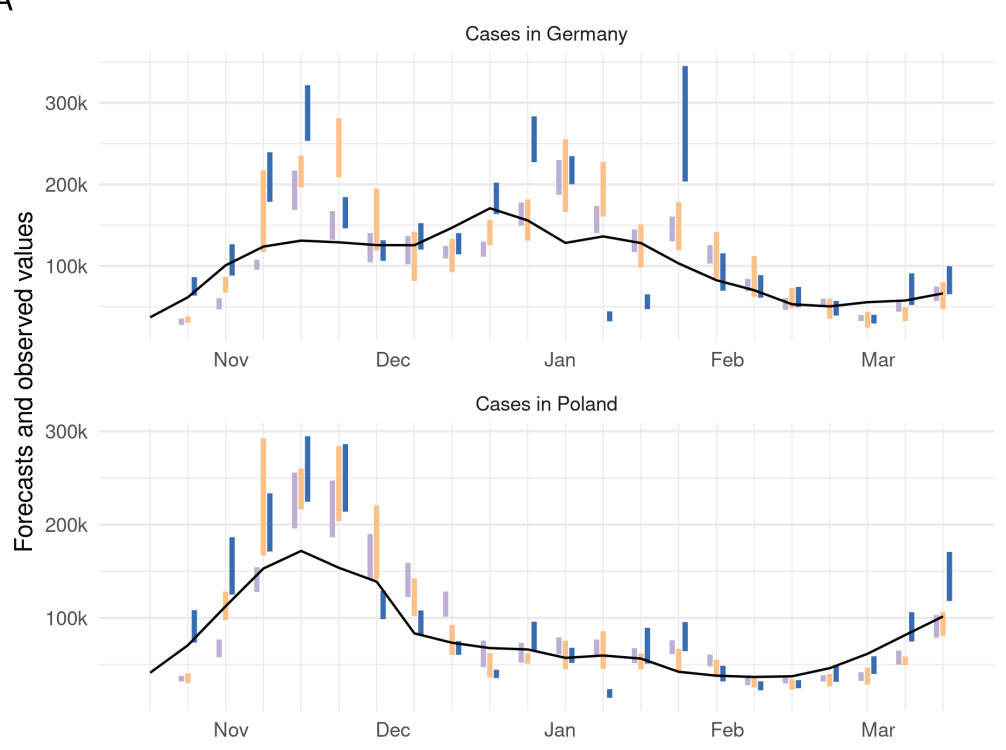

C

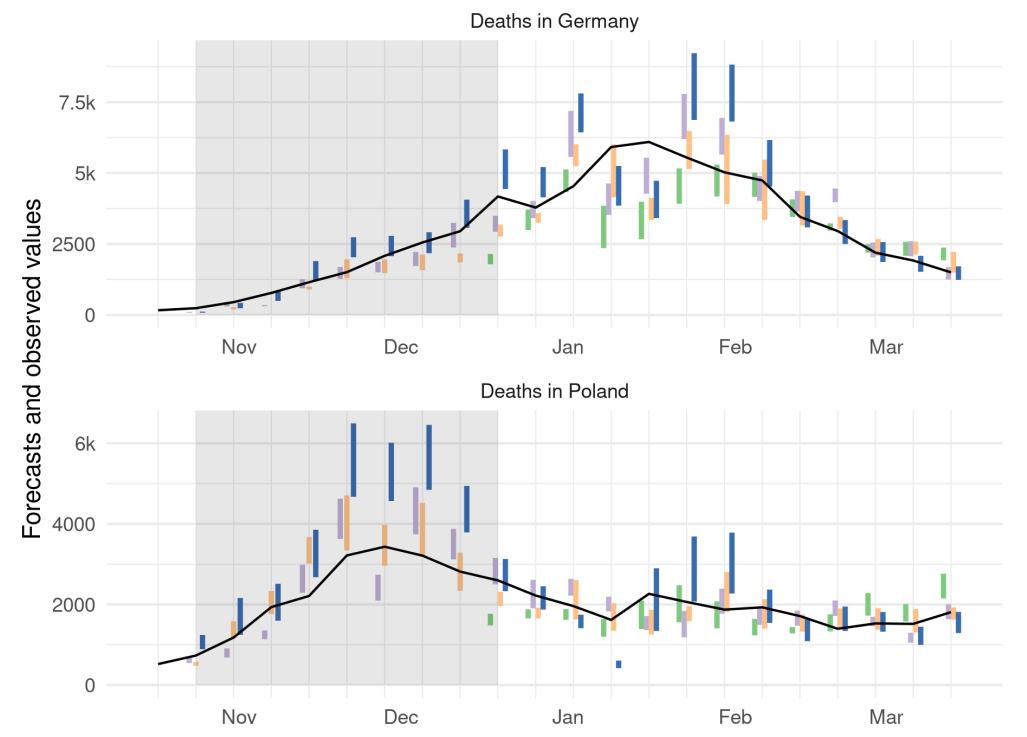

B

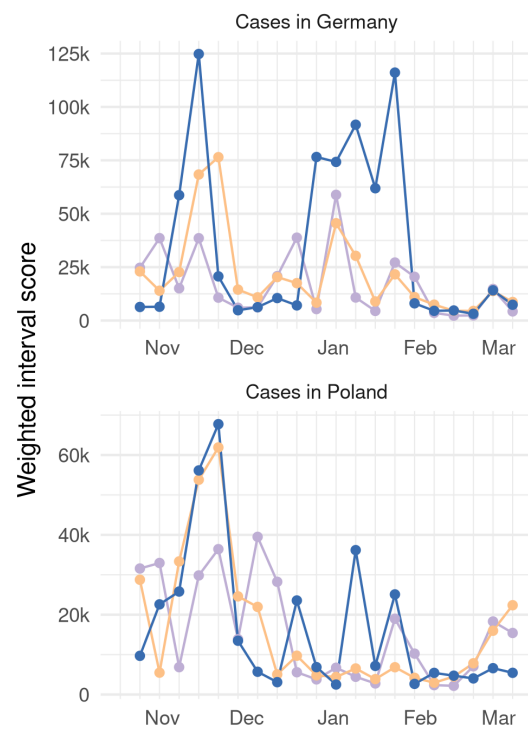

D

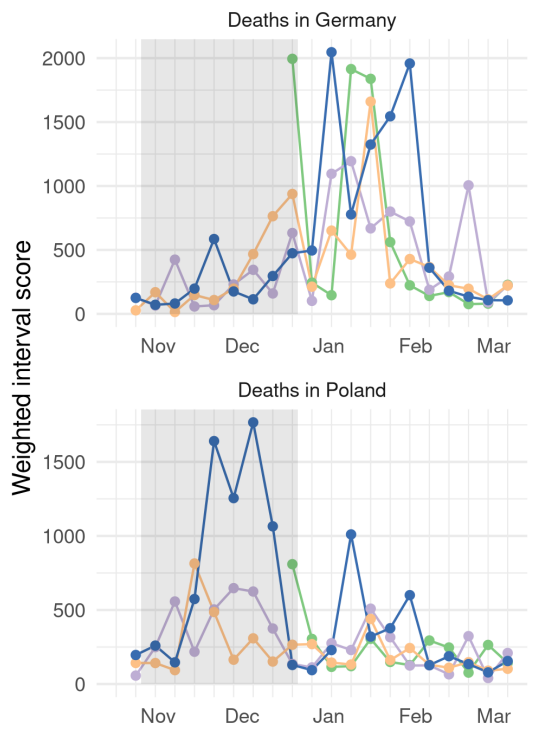

Model $\rightarrow$ Convolution $\rightarrow$ Crowd forecast $\rightarrow$ Hub-ensemble $\rightarrow$ Renewal

Figure 2: A, C: Visualisation of 50\% prediction intervals of two week ahead forecasts against the reported values. Forecasts that were not scored (because there was no complete set of death forecasts available) are greyed out. B, D: Visualisation of corresponding WIS.

\subsection{Case Forecasts}

For cases, crowd forecasts had a lower mean weighted interval score (WIS, lower values indicate better performance) than both the renewal model and the Hub ensemble across all forecast horizons (Figure 1A) and locations (Figure 7A). For two week ahead forecasts, mean WIS relative to the Hub ensemble $(=1)$ was 
0.89 for crowd forecasts and 1.40 for the renewal model (Table 2). Across all forecasting approaches, locations and forecast horizons, the distribution of WIS values was very right-skewed, and average performance was heavily influenced by outliers (Figure 3). Overall, low variance in forecast performance was closely linked with good mean performance (Figures $1 \mathrm{H}$ and and 1A), suggesting that the ability to avoid large errors was an important factor in determining overall performance. The impact of outlier values was especially pronounced for the renewal model, which had more outliers (Figure 3, as well as the highest standard deviation of WIS values (standard deviation of the WIS relative to the WIS sd of the Hub ensemble was 1.54 at the two weeks ahead horizon), while the ensemble of crowd forecasts (rel. WIS sd 0.76) and the Hub ensemble (=1) showed more stable performance.

To varying degrees, all forecasts exhibited trend-following behaviour and were rarely able to predict a change in trend before it had happened. For example, all forecasts failed to predict the change in trend from increase to decrease that happened in November in Germany and severely overshot reported cases (Figure 2A). This was most striking for the renewal model, which extrapolated unconstrained exponential growth based on the recent past of observations. The Hub ensemble and the crowd forecast, which had both been under-predicting throughout October, also failed to predict the change in trend after cases peaked, but less severely so. Human forecasters, possibly aware of the semi-lockdown announced on November 2nd 2020 (Deutsche Welle, 2020) and the change in the testing regime (with stricter test criteria) on November 11th 2020 (Ärzteblatt, 2020), were fastest to adapt to the new trend, and the Hub ensemble slowest. In December, cases rose again in Germany, with all models under-predicting this growth to varying extents. As in October, the renewal model captured the phase of exponential growth in cases slightly better than other approaches, but again overshot when reported case numbers fell over Christmas. The large variance in predictions in January in Germany (severe under-prediction followed by severe over-prediction) may in part be caused by the fact that the renewal model operated on daily data and therefore was susceptible to fluctuations in daily reporting around Christmas that would not have influenced on weekly reporting. Similar trends in performance were evident in Poland, with the crowd forecast quickest at adapting to the change in trend in November. In general, there were fewer large outlier forecasts in Poland and in particular the renewal model performed more in line with other forecasts there.

All forecasting approaches, including the Hub ensemble, were overconfident, i.e. they showed lower than nominal coverage (meaning that $50 \%(90 \%)$ prediction intervals generally covered less than $50 \%(90 \%)$ of the actually observed values) (Figure 1C and 1D). Coverage for all forecasts deteriorated with increasing forecast horizon, indicating that all forecasting approaches struggled to quantify uncertainty appropriately for case forecasts. This was especially an issue for crowd forecasts, which had markedly shorter prediction intervals (i.e., narrower and more confident predictive distributions) than other approaches (Figure 1E) and only showed a small increase in uncertainty across forecast horizons. In spite of good performance in terms of the absolute error (Figure 1G), the narrow forecast intervals led to forecasts which were severely overconfident (covering only $36 \%$ and $55 \%$ of all observations with the $50 \%$ and $90 \%$ prediction intervals of all forecasts made at a two week forecast horizon, and only $5 \%$ and $38 \%$ four weeks ahead) (Figure 1C,D and Tables 2 and 3). Despite worse performance in terms of absolute error (Figure 1G), the renewal model achieved better calibration (comparable to the Hub ensemble), as uncertainty increased rapidly across forecast horizons.

The renewal model exhibited a noticeable tendency towards over-predicting reported cases across all horizons. The crowd forecast tended to over-predict at longer forecast horizons, whereas the Hub ensemble showed no systematic bias (Figure 1F). Regardless of a general relative tendency to over-predict, all forecasting approaches incurred larger absolute penalties from over- than from under-prediction (see decomposition of the WIS into absolute penalties for over-prediction, under-prediction and dispersion in Figures 1A and 1B and Tables 2 and 3 ).

Generally, trends in overall performance were broadly similar across locations (Figures 6 and 7). Due to the differing population sizes and numbers of notifications in Germany and Poland absolute scores were difficult to compare directly. However, relative to the Hub ensemble, the crowd forecasts performed noticeably better in Germany than in Poland and the renewal model better in Poland than in Germany (Figure 7A and 7G). 


\subsection{Death Forecasts}

For deaths, the Hub ensemble outperformed the crowd forecasts as well as our model-based approaches across all forecast horizons and locations (Figure 1B, Figure 6B). Relative WIS values for the models two weeks ahead were 1.22 (convolution model), 1.26 (crowd forecast), 1 (Hub ensemble) and 1.79 (renewal model). The crowd forecasts performed better than the renewal model across all forecast horizons and locations (Figure 1B, Figure 6B), and also better than the convolution model three and four weeks ahead. Poor performance of the renewal model, especially at longer horizons, indicates that an approach that does not know about past cases, but instead estimates and projects a separate $R_{t}$ trace from deaths, does not use the available information efficiently. The convolution model was able to outperform both the renewal model and the crowd forecasts at shorter forecast horizons (where the delay between cases and deaths means that future deaths are largely informed by present cases), but saw performance deteriorate at three and four weeks ahead (where case predictions from the renewal model were increasingly used to inform death predictions) (Figure 1B, Table 3).

As past cases and hospitalisations can be used as predictors, predicting a change in trend may be easier for deaths than for cases. Even though all forecasts generally struggled with this, there were some instances where changing trends were well captured or even anticipated. In Poland, for example, the Hub ensemble was able to capture or even anticipate the peak in deaths in December quite well (whereas the renewal model and crowd forecast did not). The renewal model, which mostly exhibited trend-following behaviour, correctly predicted another increase in weekly deaths in mid-January (potentially based on changes in daily deaths, as the renewal model did not know about past cases). In Germany in early January, all models predicted a decrease in deaths two to three weeks before it actually happened. Predictions from the renewal model at that time were likely strongly influenced by an unexpected drop in reported deaths in December. The other forecasting approaches and in particular, the convolution model may have been affected by potentially under-reported case numbers around Christmas. When the decrease that all models had predicted to happen in early January failed to materialise, the renewal model and the crowd forecast noticeably over-corrected and over-predicted deaths in the following weeks, while the Hub ensemble, and to a slightly lesser degree, the convolution model were able to capture the downturn well when it finally happened at the end of January.

Death forecasts, generally, showed greater coverage of the $50 \%$ and $90 \%$ prediction intervals than case forecasts and no decrease in coverage across forecast horizons, indicating that it might be easier to appropriately quantify uncertainty for death forecasts. The Hub ensemble had the greatest coverage, with empirical coverage of the $50 \%$ and $90 \%$ prediction intervals exceeding $50 \%$, and $90 \%$, respectively, across all forecast horizons. Coverage for the crowd forecasts and our model-based approaches was generally lower than that of the Hub ensemble and mostly slightly lower than nominal coverage (Figure 1C and 1D). As for cases, the crowd forecast tended to have the narrowest prediction intervals and uncertainty increased most slowly across forecast horizons, and the renewal model forecasts generally were widest. The convolution model had relatively narrow prediction intervals for short forecast horizons, but had rapidly (and non-linearly) increasing uncertainty for longer forecast horizons, driven by increasing uncertainty in the underlying case forecasts.

For deaths, the ensemble of crowd forecasts had a consistent tendency to over-predict $1 \mathrm{~F}$. The convolution model had a strong tendency to under-predict, which steadily decreased for longer forecast horizons. The renewal model (which over-predicted for cases) and the Hub ensemble slightly tended towards under-prediction. For deaths, absolute over- and under-prediction penalties were more in line with a general relative tendency to over- or under-predict than for cases (Figure 1A, 1B and Tables 2, 3). 
A
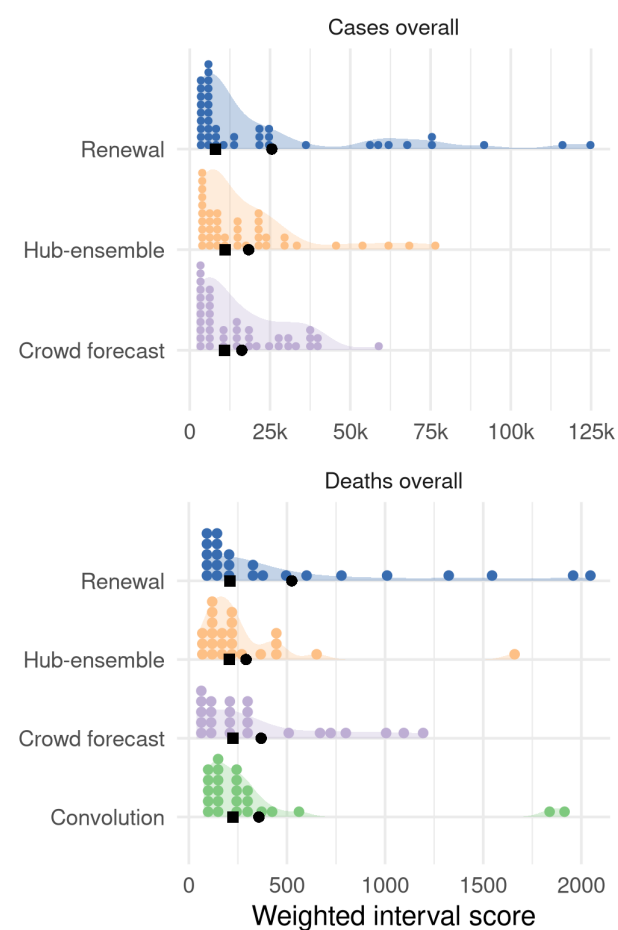

Model Convolution

B
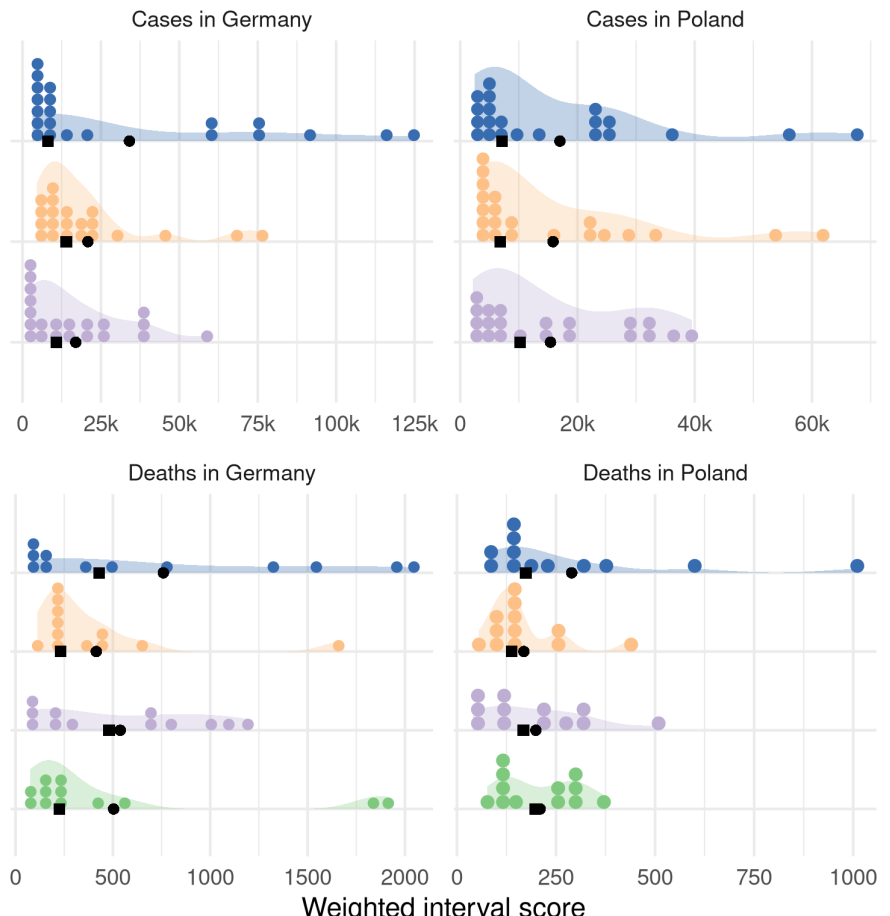

Weighted interval score

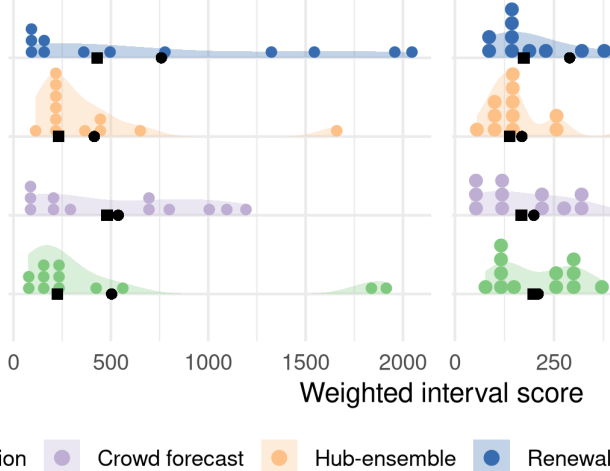

Figure 3: A: Distribution of weighted interval scores for two week ahead forecasts of the different models and forecast targets. Points denote single forecasts scores, while the shaded area shows an estimated probability density. B: Distribution of WIS separate by country. 
A

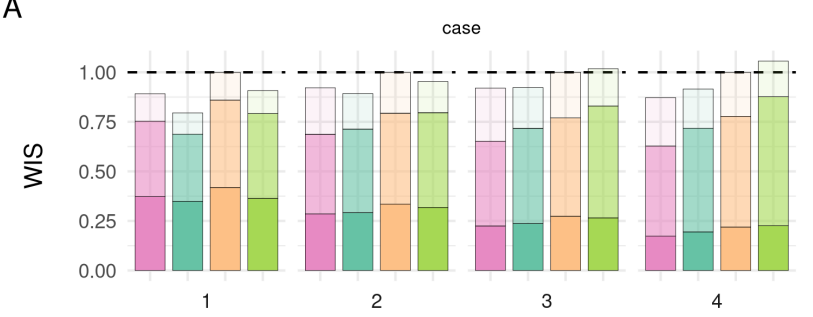

C

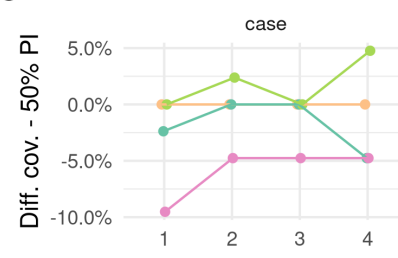

E

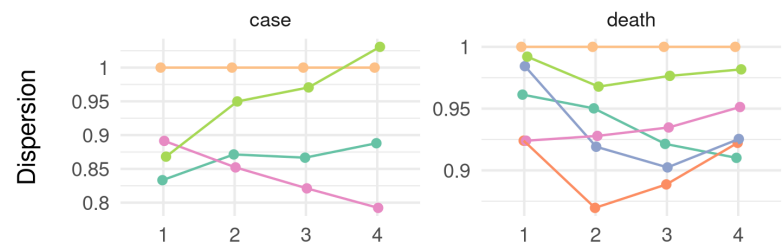

G

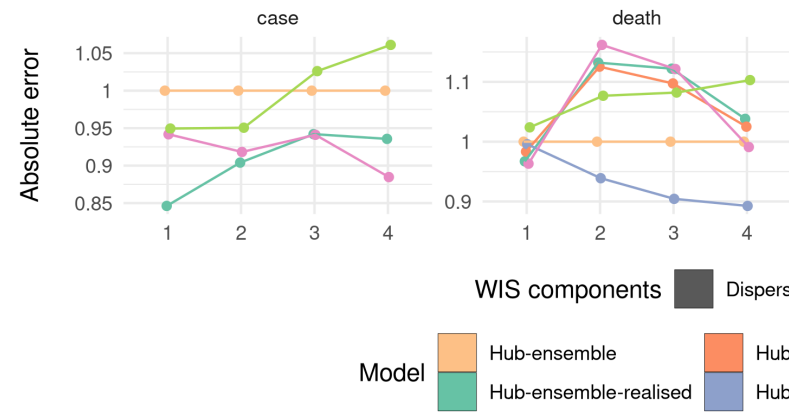

B

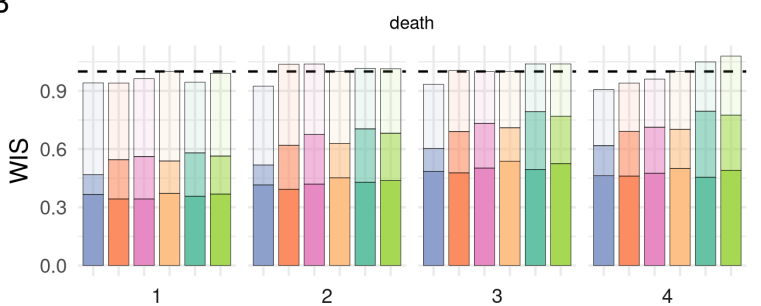

$\mathrm{D}$

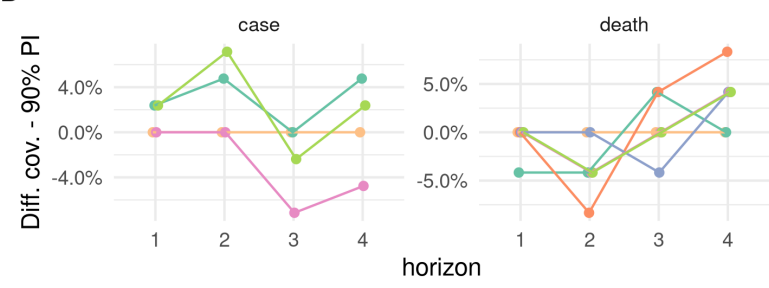

$\mathrm{F}$

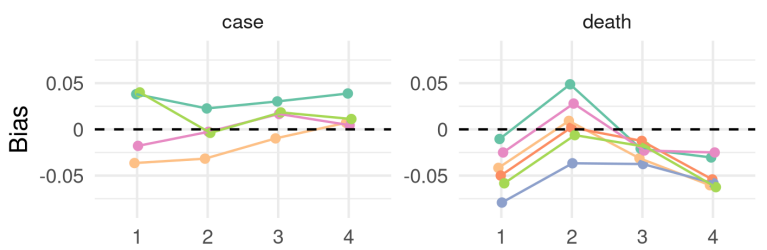

$\mathrm{H}$

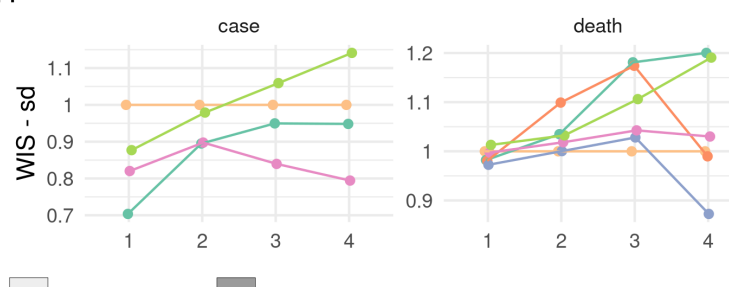

Figure 4: Visualisation of aggregate performance metrics across forecast horizons for the different versions of the Hub median ensemble. "Hub-ensemble" excludes all our models, Hub-ensemble-all includes all of our models, "Hub-ensemble-real" is the real hub-ensemble with the renewal model and the crowd forecasts included. Values (except for Bias) are computed as differences to the Hub ensemble excluding our contributions. For Coverage, this is an absolute difference, for other metrics this is a percentage difference. A: mean weighted interval score (WIS) across horizons. B: median WIS. C: Absolute error of the median forecast. D: Standard deviation of the WIS. E: Dispersion (higher values mean greater spread of the forecast). F: Bias, i.e. general tendency to over- or underpredict. Values are between -1 (complete under-prediction) and 1 (complete over-prediction) and 0 ideally. G: Empirical coverage of the $50 \%$ prediction intervals. F: Empirical coverage of the $90 \%$ prediction intervals

\subsection{Contribution to the Forecast Hub}

Of our three models, only the renewal model and the crowd forecast were included in the official Forecast Hub median ensemble ("hub-ensemble-realised"), while the convolution model was never included as it was deemed too similar to the existing renewal model. In the official Hub ensemble, there were on average 7.1 models 
included (including our own), with a median of 7, a minimum of 4 (on 28 December 2020 over the Christmas period) and a maximum of 10. Versions that included either all of our models ("hub-ensemble-with-all") or only one of them ("hub-ensemble-with-X") were computed retrospectively. An overview of all models and ensemble versions is shown in Table 10 in the SI.

For cases, our contributions (compared to the Hub ensemble without our contributions) consistently improved performance across all forecasting horizons (rel. WIS 0.9 two weeks ahead, Table 4). Contributions from the crowd forecasts alone also improved performance of the Hub ensemble across all forecast horizons, while contributions from the renewal model had a negative effect for longer horizons (rel. WIS 1.02 three weeks ahead, 1.06 four weeks ahead). The realised ensemble including both models performed better or equal compared to all versions with only one model included for up to three weeks ahead, suggesting synergistic effects. Only for predictions four weeks ahead would removing the renewal model have improved performance (Table 5). The realised ensemble performed comparably to the crowd forecasts for predictions one to two weeks ahead, and worse for greater forecast horizons.

For deaths, contributions from the renewal model and crowd forecast together improved performance only for one week ahead predictions and showed an increasingly negative impact on performance for longer horizons (rel. WIS of the hub-ensemble-realised 1.01 two weeks ahead, 1.05 four weeks ahead, Tables 4 and 5). Individual contributions from both the renewal model and the crowd forecast were largely negative, while a version of the Hub ensemble with only the convolution model included would have performed consistently better across all forecast horizons (with the positive impact increasing for longer horizons). This is especially interesting as the convolution model performed consistently worse than the pre-existing Hub ensemble (Figure 1) and especially worse for longer horizons.

We also considered the impact of our contributions on a version of the Hub ensemble constructed by taking the quantile-wise mean, rather than the median. General trends were similar, with the notable exception of the convolution model, which had a consistently positive impact on the median ensemble, but a mixed and mostly slightly negative impact on the mean ensemble (Figures 4B and 19B). This may happen if a model is more correct directionally relative to the pre-existing ensemble, but overshoots in absolute terms, thereby moving the ensemble too far. For both the mean and the median ensemble, changes in performance from adding or removing models were of a similar order of magnitude, suggesting that at least in this instance, with a relatively small ensemble size, the median ensemble was not necessarily more 'robust' to changes than the mean ensemble. However, the ensemble version with all our forecasts included ("hub-ensemble-with-all") tended to perform relatively better for the median ensemble than the mean ensemble, suggesting that adding more models may be more beneficial or 'safer' for the median than for the mean ensemble as directional errors can more easily cancel out than errors in absolute terms.

\section{Discussion}

Epidemiological forecasting modelling combines knowledge about infectious disease dynamics with the subjective opinion of the researcher who develops and refines the model. In this study, we compared forecasts of cases of and deaths from COVID-19 in Germany and Poland based purely on human judgement and elicited from a crowd of researchers and volunteers against forecasts from two semi-mechanistic epidemiological models. In spite of the small number of participants and a general tendency to be overconfident, crowd forecasts consistently outperformed our epidemiological models as well as the Hub ensemble when forecasting cases but not when forecasting deaths. This suggests that humans might be relatively good at foreseeing trends that are hard to model but may struggle to form an intuition for the exact relationship between cases and deaths.

Past studies have evaluated the performance of model-based forecasting approaches as well as human experts and non-experts in various contexts. However, most of these studies either focused only on the evaluation of (expert-tuned) model-based approaches (Cramer et al., 2021; Cramer et al., 2020; e.g. Funk et al., 2020), or exclusively on human forecasts (Atanasov et al., 2016; McAndrew \& Reich, 2020; Recchia et al., 2021; Tetlock et al., 2014). In contrast, we directly compared human and model-based forecasts. This is similar to the approach taken by Farrow et al. (2017), but extends it in several ways. While Farrow et al. only asked for 
point predictions and constructed a predictive distribution from these, we asked participants to provide a full predictive distribution, allowing us to compare human forecasts and models without any further assumptions, as well as to analyse how humans quantified their uncertainty. In addition, we compared crowd forecasts to two semi-mechanistic models informed by basic epidemiological knowledge of COVID-19, allowing us to assess not only relative performance but also to analyse qualitative differences between human judgement and model-based insight. In terms of interpretability of the results, exact knowledge of our two models, as well as focus on a limited set of targets and locations was a major advantage of our study compared to larger studies conducted by the Forecast Hubs (Bracher, Wolffram, Deuschel, Görgen, Ketterer, Ullrich, Abbott, Barbarossa, Bertsimas, Bhatia, Bodych, Bosse, Burgard, Castro, et al., 2021; Cramer et al., 2021; Cramer et al., 2020; European Covid-19 Forecast Hub, 2021; Funk et al., 2020).

The strong performance of crowd forecasts in our study is in line with results from Farrow et al. who also report strong performance of human predictions in past Flu challenges despite difficulties to recruit a large number of participants. The advantage of crowd forecasts we observed over our semi-mechanistic models is likely in part explained by the fact that we compared an ensemble of crowd forecasts with single models. However, this probably explains only part of the difference, and performance relative to the Hub ensemble strongly suggests that human insight is valuable when forecasting highly volatile and potentially hard-to-predict quantities such as case numbers. One potential explanation is that humans can have access to data that is not available to or hard to integrate into model-based forecasts. Relatively good performance of our semi-mechanistic models short-term, but not longer-term, suggests that model-based forecasts are helpful to extrapolate from current conditions, but require some form of human intervention or additional assumptions to inform forecasts when conditions change over time. This human intervention may be particularly important when dealing with artefacts in reporting and data anomalies (and especially when using daily, rather than weekly data). The large variance in predictions in January in Germany for example (severe under-prediction followed by severe over-prediction, see Figure 2A), may in part be caused by the fact that the renewal model operated on daily data and therefore was susceptible to fluctuations in daily reporting around Christmas that would not have influenced on weekly reporting.

Our results suggest that human intervention may be less beneficial when forecasting deaths (especially at shorter horizons, when deaths are largely dependent on already observed cases), which benefits from the ability to model the delays and exact epidemiological relationships between different leading and lagged indicators. Relatively good performance of the convolution model, especially compared to the poor performance of the renewal model on deaths (which used only deaths to estimate and predict the effective reproduction number) underlines the importance of including leading indicators such as cases as a predictor for deaths.

Given the low number of participants in our study, it is difficult to generalise conclusions about crowd predictions to other settings. Using $\mathrm{R}$ shiny as a platform for the web application arguably created some limits to user experience and performance, influencing the number of participants and potentially creating a self-selection effect. Motivating forecasters to contribute regularly proved challenging, especially given that the majority of our participants were from the UK and may not have been familiar with all relevant details of the situation in Germany and Poland. On the other hand, R shiny facilitated quick development and allowed us to provide our crowd forecasting tooling as an open source $\mathrm{R}$ package, meaning that it is available for others to use, for example in settings like early-stage outbreaks where model-based forecasts are not available.

Our work suggests that crowd forecasts and model-based forecasts have different strengths and may be able to complement each other. When choosing a suitable approach for a given task it is important to take into account how the output will be used. In this work we focused on forecasts (which aim to predict future data points whilst accounting for all factors that might influence them), whereas policy makers might be more interested in projections (which show what would happen in the absence of any events that could change the trend) or scenario modelling. Forecasts may not be a suitable basis for informing policy decisions, if forecasters already have factored in the expectation of a future intervention. Model-based approaches can be either forecasts or projections depending on the assumptions, whereas eliciting projections that are not influenced by implicit assumptions about the future from humans may be harder.

Further work should explore the effects of humans refining their mathematical models or changing model outputs in more detail. Model-based forecasts could be used as an input to human judgement, with 
researchers adjusting predictions generated by models. Seeing a model-based forecast could help humans calibrate uncertainty better, while allowing for manual intervention to adapt spurious trend predictions. Tools need to be developed to facilitate this process at a larger scale. Human insight could also be used as an input to models. Such a 'hybrid' forecasting approach could for example ask humans to predict the trend of the effective reproduction number $R_{t}$ or the doubling rate (i.e. how the epidemic evolves) into the future and use this to estimate the exact number of cases, hospitalisations or deaths this would imply. In light of severe overconfidence, yet good performance in terms of the absolute error, post-processing of human forecasts to adjust and widen prediction intervals may be another promising approach. Crowd forecasting in general could benefit greatly from the availability of tools suitable to appeal to a greater audience. Given the good performance we and previous authors observed in spite of the limited resources available and the small number of participants, this seems worthwhile to further develop and explore. 


\section{Acknowledgements}

NIB received funding from the Health Protection Research Unit (grant code NIHR200908). SA's work was funded by the Wellcome Trust (grant: 210758/Z/18/Z). The work of JB was supported by the Helmholtz Foundation via the SIMCARD Information and Data Science Pilot Project. This research was partly funded by the National Institute for Health Research (NIHR) (16/137/109 \& 16/136/46) using UK aid from the UK Government to support global health research. The views expressed in this publication are those of the author(s) and not necessarily those of the NIHR or the UK Department of Health and Social Care. BJQ is supported in part by a grant from the Bill and Melinda Gates Foundation (OPP1139859). EvL acknowledges funding by the National Institute for Health Research (NIHR) Health Protection Research Unit (HPRU) in Modelling and Health Economics (grant number NIHR200908) and the European Union's Horizon 2020 research and innovation programme - project EpiPose (101003688). AC acknowledges funding by the NIHR, the Sergei Brin foundation, USAID, and the Academy of Medical Sciences. SF's work was supported by the Wellcome Trust (grant: 210758/Z/18/Z), and the NIHR (NIHR200908).

We thank all forecasters who participated in this study for their contribution.

We would also like to acknowledge (in a randomised order) the members of Centre for the Mathematical Modelling of Infectious Diseases COVID-19 Working Group at the the London School of Hygiene \& Tropical Medicine: Oliver Brady, Katharine Sherratt, Kaja Abbas, Kerry LM Wong, Charlie Diamond, Katherine E. Atkins, Rein M G J Houben, Jiayao Lei, Rachel Lowe, David Simons, Sophie R Meakin, Nicholas G. Davies, Timothy W Russell, Kevin van Zandvoort, Quentin J Leclerc, Kathleen O'Reilly, Stéphane Hué, Alicia Rosello, Emilie Finch, C Julian Villabona-Arenas, Thibaut Jombart, W John Edmunds, Yalda Jafari, Jack Williams, Alicia Showering, Damien C Tully, Jon C Emery, Carl A B Pearson, David Hodgson, Frank G Sandmann, Petra Klepac, Adam J Kucharski, Graham Medley, Yang Liu, Simon R Procter, Emily S Nightingale, William Waites, Rosanna C Barnard, Joel Hellewell, Yung-Wai Desmond Chan, Fiona Yueqian Sun, Hamish P Gibbs, Rosalind M Eggo, Lloyd A C Chapman, Stefan Flasche, James W Rudge, Akira Endo, Naomi R Waterlow, Paul Mee, James D Munday, Ciara V McCarthy, Mihaly Koltai, Amy Gimma, Christopher I Jarvis, Megan Auzenbergs, Matthew Quaife, Fabienne Krauer, Samuel Clifford, Georgia R Gore-Langton, Arminder K Deol, Kiesha Prem, Gwenan M Knight, Rachael Pung, Anna M Foss. 


\section{A Supplementary information}

\section{A.1 Scoring metrics used}

Table 1: Overview of the scoring metrics used.

\begin{tabular}{|c|c|}
\hline Metric & Explanation \\
\hline \multirow[t]{6}{*}{$\begin{array}{l}\text { WIS (Weighted) } \\
\text { interval score }\end{array}$} & $\begin{array}{l}\text { The weighted interval score (smaller values are better) is a proper scoring rule for } \\
\text { quantile forecasts. It converges to the continuos ranked probability score (which itself } \\
\text { is a generalisation of the absolute error to probabilistic forecasts) for an increasing } \\
\text { number of intervals. The score can be decomposed into a dispersion (uncertainty) } \\
\text { component and penalties for over- and underprediction. For a single interval, the } \\
\text { score is computed as }\end{array}$ \\
\hline & $I S_{\alpha}(F, y)=(u-l)+\frac{2}{\alpha} \cdot(l-y) \cdot 1(y \leq l)+\frac{2}{\alpha} \cdot(y-u) \cdot 1(y \geq u)$ \\
\hline & $\begin{array}{l}\text { where } 1() \text { is the indicator function, } y \text { is the true value, and } l \text { and } u \text { are the } \frac{\alpha}{2} \text { and } \\
1-\frac{\alpha}{2} \text { quantiles of the predictive distribution } F \text {, i.e. the lower and upper bound of a } \\
\text { single prediction interval. For a set of } K \text { prediction intervals and the median } m \text {, the } \\
\text { score is computed as a weighted sum, }\end{array}$ \\
\hline & $W I S=\frac{1}{K+0.5} \cdot\left(w_{0} \cdot|y-m|+\sum_{k=1}^{K} w_{k} \cdot I S_{\alpha}(F, y)\right)$ \\
\hline & where $w_{k}$ is a weight for every interval. Usually, $w_{k}=\frac{\alpha_{k}}{2}$ and $w_{0}=0.5$ \\
\hline & $\begin{array}{l}\text { Its proximity to the absolute error means that when averaging across multiple targets } \\
\text { (e.g. different weeks), it will be dominated by targets with higher absolute values. }\end{array}$ \\
\hline $\begin{array}{l}\text { Interval } \\
\text { coverage }\end{array}$ & $\begin{array}{l}\text { Interval coverage is a measure of marginal calibration and indicates the proportion of } \\
\text { observed values that fall in a given prediction interval range. Nominal coverage } \\
\text { represents the percentage of observed values that should ideally be covered (e.g. we } \\
\text { would like a } 50 \text { percent prediction interval to cover on average } 50 \text { percent of the } \\
\text { observations), while empirical coverage is the actual percentage of observations } \\
\text { covered by a certain prediction interval. }\end{array}$ \\
\hline \multirow[t]{6}{*}{ Bias } & $\begin{array}{l}\text { (Relative) bias is a measure of the general tendency of a forecaster to over- or } \\
\text { underpredict. Values are between }-1 \text { and } 1 \text { and } 0 \text { ideally. For continuous forecasts, } \\
\text { bias is given as }\end{array}$ \\
\hline & $B(F, y)=1-2 \cdot(F(y))$ \\
\hline & where $F$ is the CDF of the predictive distribution and $y$ is the observed value. \\
\hline & $\begin{array}{l}\text { For quantile forecasts, } F(y) \text { is replaced by a quantile rank. The appropriate quantile } \\
\text { rank is determined by whether the median forecast is below or above the true value. }\end{array}$ \\
\hline & $\begin{array}{l}\text { We then take the innermost quantile rank for which the quantile is still larger } \\
\text { (under-prediction) or smaller (over-prediction) than the observed value. }\end{array}$ \\
\hline & $\begin{array}{l}\text { In contrast to the over- and underprediction penalties of the interval score it is bound } \\
\text { between } 0 \text { and } 1 \text { and represents a general tendency of forecasts to be biased rather } \\
\text { than the absolute amount of over- and underprediction. It is therefore a more robust } \\
\text { measurement. }\end{array}$ \\
\hline
\end{tabular}


medRxiv preprint doi: https://doi.org/10.1101/2021.12.01.21266598; this version posted December 5, 2021. The copyright holder for this preprint (which was not certified by peer review) is the author/funder, who has granted medRxiv a license to display the preprint in perpetuity.

It is made available under a CC-BY 4.0 International license.

\section{A.2 The crowdforecasting app}

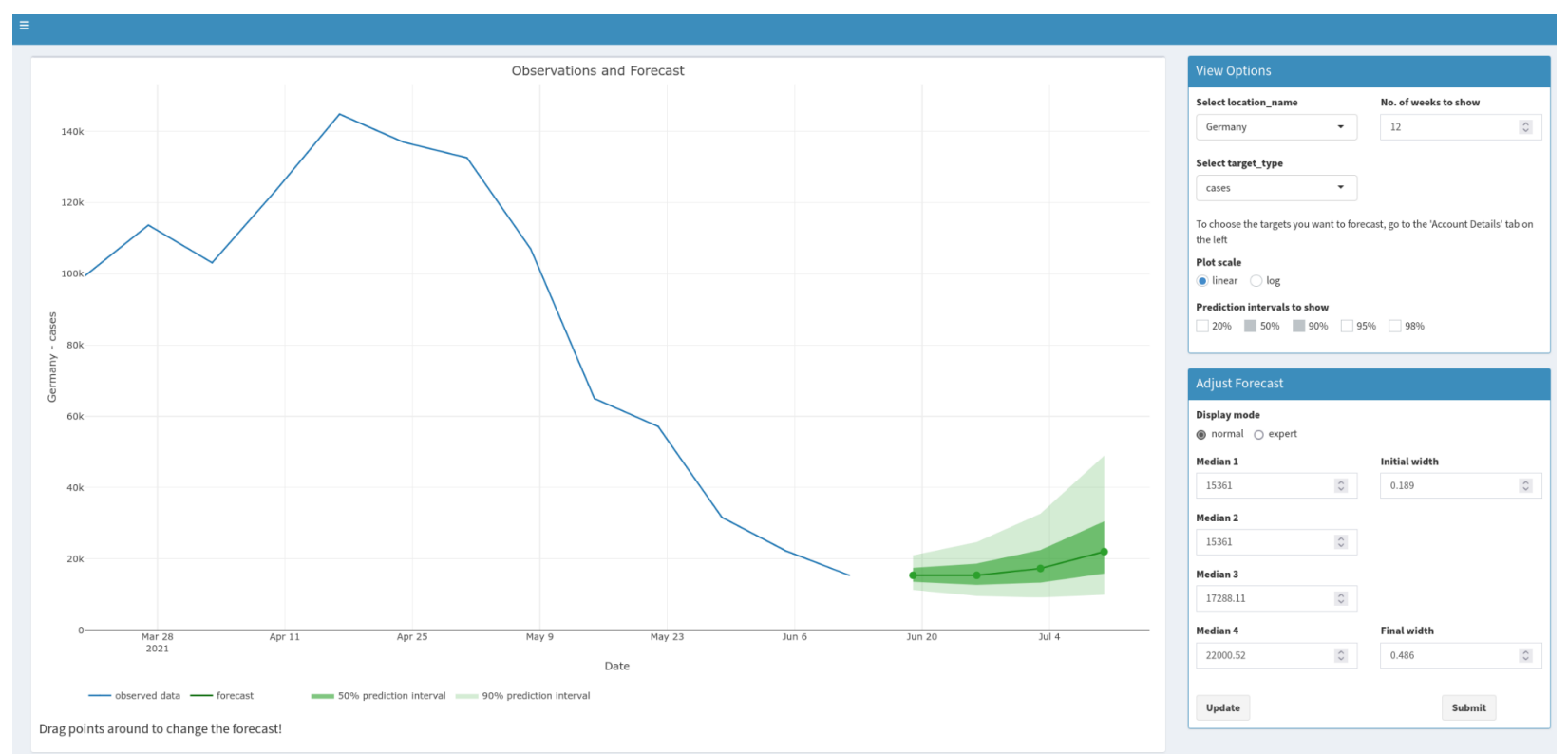

Figure 5: Screenshot of the crowdforecasting app used to elicit predictions (made in June 2021). 


\section{A.3 Further details on the semi-mechanistic forecasting models}

\section{A.3.1 Renewal equation model}

The model was initialised prior to the first observed data point by assuming constant exponential growth for the mean of assumed delays from infection to case report.

$$
\begin{aligned}
I_{t} & =I_{0} \exp (r t) \\
I_{0} & \sim \mathcal{L N}\left(\log I_{o b s}, 0.2\right) \\
r & \sim \mathcal{L N}\left(r_{o b s}, 0.2\right)
\end{aligned}
$$

Where $I_{o b s}$ and $r_{o b s}$ are estimated from the first week of observed data. For the time window of the observed data infections were then modelled by weighting previous infections by the generation time and scaling by the instantaneous reproduction number. These infections were then convolved to cases by date $\left(O_{t}\right)$ and cases by date of report $\left(D_{t}\right)$ using log-normal delay distributions. This model can be defined mathematically as follows,

$$
\begin{aligned}
\log R_{t} & =\log R_{t-1}+\mathrm{GP}_{t} \\
I_{t} & =R_{t} \sum_{\tau=1}^{15} w\left(\tau \mid \mu_{w}, \sigma_{w}\right) I_{t-\tau} \\
O_{t} & =\sum_{\tau=0}^{15} \xi_{O}\left(\tau \mid \mu_{\xi_{O}}, \sigma_{\xi_{O}}\right) I_{t-\tau} \\
D_{t} & =\alpha \sum_{\tau=0}^{15} \xi_{D}\left(\tau \mid \mu_{\xi_{D}}, \sigma_{\xi_{D}}\right) O_{t-\tau} \\
C_{t} & \sim \operatorname{NB}\left(\omega_{(t \bmod 7)} D_{t}, \phi\right)
\end{aligned}
$$

Where,

$$
\begin{aligned}
w & \sim \mathcal{G}\left(\mu_{w}, \sigma_{w}\right) \\
\xi_{O} & \sim \mathcal{L N}\left(\mu_{\xi_{O}}, \sigma_{\xi_{O}}\right) \\
\xi_{D} & \sim \mathcal{L N}\left(\mu_{\xi_{D}}, \sigma_{\xi_{D}}\right)
\end{aligned}
$$

This model used the following priors for cases,

$$
\begin{aligned}
R_{0} & \sim \mathcal{L N}(0.079,0.18) \\
\mu_{w} & \sim \mathcal{N}(3.6,0.7) \\
\sigma_{w} & \sim \mathcal{N}(3.1,0.8) \\
\mu_{\xi_{O}} & \sim \mathcal{N}(1.62,0.064) \\
\sigma_{\xi_{O}} & \sim \mathcal{N}(0.418,0.069) \\
\mu_{\xi_{D}} & \sim \mathcal{N}(0.614,0.066) \\
\sigma_{\xi_{D}} & \sim \mathcal{N}(1.51,0.048) \\
\alpha & \sim \mathcal{N}(0.25,0.05) \\
\frac{\omega}{7} & \sim \operatorname{Dirichlet}(1,1,1,1,1,1,1) \\
\phi & \sim \frac{1}{\sqrt{\mathcal{N}(0,1)}}
\end{aligned}
$$


and updated the reporting process as follows when forecasting deaths,

$$
\begin{aligned}
\mu_{\xi_{D}} & \sim \mathcal{N}(2.29,0.076) \\
\sigma_{\xi_{D}} & \sim \mathcal{N}(0.76,0.055) \\
\alpha & \sim \mathcal{N}(0.005,0.0025)
\end{aligned}
$$

$\alpha, \mu, \sigma$, and $\phi$ were truncated to be greater than 0 and with $\xi$, and $w$ normalised to sum to 1.

The prior for the generation time was sourced from (Ganyani et al., 2020) but refit using a log-normal incubation period with a mean of 5.2 days (SD 1.1) and SD of 1.52 days (SD 1.1) with this incubation period also being used as a prior (Lauer et al., 2020) for $\xi_{O}$. This resulted in a gamma-distributed generation time with mean 3.6 days (standard deviation (SD) 0.7), and SD of 3.1 days (SD 0.8) for all estimates. We estimated the delay between symptom onset and case report or death required to convolve latent infections to observations by fitting an integer adjusted log-normal distribution to 10 subsampled bootstraps of a public linelist for cases in Germany from April 2020 to June 2020 with each bootstrap using 1\% or 1769 samples of the available data (Abbott, Sherratt, et al., 2020; Xu et al., 2020) and combining the posteriors for the mean and standard deviation of the log-normal distribution (Abbott, Hellewell, Hickson, et al., 2020; epiforecasts.io/covid, 2020; Evaluating the Use of the Reproduction Number as an Epidemiological Tool, Using Spatio-Temporal Trends of the Covid-19 Outbreak in England / medRxiv, n.d.; Stan Development Team, 2020).

$G P_{t}$ is an approximate Hilbert space Gaussian process as defined in (Riutort-Mayol et al., 2020) using a Matern 3/2 kernel using a boundary factor of 1.5 and 17 basis functions (20\% of the number of days used in fitting). The length scale of the Gaussian process was given a log-normal prior with a mean of 21 days, and a standard deviation of 7 days truncated to be greater than 3 days and less than 60 days. The magnitude of the Gaussian process was assumed to be normally distributed centred at 0 with a standard deviation of 0.1.

From the forecast time horizon $(T)$ and onwards the last value of the Gaussian process was used (hence $R_{t}$ was assumed to be fixed) and latent infections were adjusted to account for the proportion of the population that was susceptible to infection as follows,

$$
I_{t}=\left(N-I_{t-1}^{c}\right)\left(1-\exp \left(\frac{-I_{t}^{\prime}}{N-I_{T}^{c}}\right)\right)
$$

where $I_{t}^{c}=\sum_{s<t} I_{s}$ are cumulative infections by $t-1$ and $I_{t}^{\prime}$ are the unadjusted infections defined above. This adjustment is based on that implemented in the epidemia $\mathrm{R}$ package (Bhatt et al., n.d.; Scott et al., 2020).

A.3.1.1 Convolution model The convolution model shares the same observation model as the renewal model but rather than assuming that an observation is predicted by itself using the renewal equation instead assumes that it is predicted entirely by another observation after some parametric delay. It can be defined mathematically as follows,

$$
D_{t} \sim \mathrm{NB}\left(\omega_{(t \quad \bmod 7)} \alpha \sum_{\tau=0}^{30} \xi(\tau \mid \mu, \sigma) C_{t-\tau}, \phi\right)
$$

with the following priors, 


$$
\begin{aligned}
\frac{\omega}{7} & \sim \operatorname{Dirichlet}(1,1,1,1,1,1,1) \\
\alpha & \sim \mathcal{N}(0.01,0.02) \\
\xi & \sim \mathcal{L N}(\mu, \sigma) \\
\mu & \sim \mathcal{N}(2.5,0.5) \\
\sigma & \sim \mathcal{N}(0.47,0.2) \\
\phi & \sim \frac{1}{\sqrt{\mathcal{N}(0,1)}}
\end{aligned}
$$

with $\alpha, \mu, \sigma$, and $\phi$ truncated to be greater than 0 and with $\xi$ normalised such that $\sum_{\tau=0}^{30} \xi(\tau \mid \mu, \sigma)=1$.

\section{A.3.2 Model fitting}

Both models were implemented using the EpiNow2 R package (version 1.3.3) (Abbott, Hellewell, Hickson, et al., 2020). Each forecast target was fitted independently for each model using Markov-chain Monte Carlo (MCMC) in stan (Stan Development Team, 2020). A minimum of 4 chains were used with a warmup of 250 samples for the renewal equation-based model and 1000 samples for the convolution model. 2000 samples total post warmup were used for the renewal equation model and 4000 samples for the convolution model. Different settings were chosen for each model to optimise compute time contingent on convergence. Convergence was assessed using the R hat diagnostic (Stan Development Team, 2020). For the convolution model forecast the case forecast from the renewal equation model was used in place of observed cases beyond the forecast horizon using 1000 posterior samples. 12 weeks of data was used for both models though only 3 weeks of data were included in the likelihood for the convolution model. 


\section{A.4 Tables with results of the forecast evaluation}

Table 2: Scores for one and two week ahead forecasts (cut to three significant digits and rounded). Note that scores for cases (which include the whole period from October 12th 2020 until March 1st 2021) and deaths (which include only forecasts from the 21st of December 2020 on) are computed on different subsets. Numbers in brackets show the metrics relative to the Hub ensemble (i.e. the median ensemble of all other models submitted to the German and Polish Forecast Hub, excluding our contributions). WIS is the mean weighted interval score (lower values are better), WIS - sd is the standard deviation of all scores achieved by a model. Dispersion, over-prediction and under-prediction together sum up to the weighted interval score. Bias (between -1 and 1, 0 is ideal) represents the general average tendency of a model to over- or underpredict. 50\% and 90\%coverage are the percentage of observed values that fell within the $50 \%$ and $90 \%$ prediction intervals of a model.

\begin{tabular}{|c|c|c|c|c|c|c|c|c|c|c|}
\hline & Model & WIS & WIS - sd & dispersion & Underpred. & Overpred. & Bias & Abs. error & $50 \%$-Cov. & $90 \%-$ Cov. \\
\hline \multicolumn{11}{|l|}{ Cases } \\
\hline \multirow{3}{*}{1 wk ahead } & Crowd forecast & $7010(0.8)$ & $7480(0.64)$ & $2680(0.73)$ & $1700(1.38)$ & $2630(0.68)$ & -0.01 & $10400(0.82)$ & 0.55 & 0.79 \\
\hline & Hub-ensemble & $8770(1)$ & $11700(1)$ & $3670(1)$ & $1230(1)$ & $3870(1)$ & -0.04 & $12700(1)$ & 0.57 & 0.81 \\
\hline & Renewal & $8740(1)$ & $11800(1.01)$ & $2190(0.6)$ & $2720(2.21)$ & $3830(0.99)$ & 0.18 & $12000(0.94)$ & 0.48 & 0.71 \\
\hline \multirow{3}{*}{2 wk ahead } & Crowd forecast & $16200(0.89)$ & $16600(0.76)$ & $3660(0.6)$ & $5930(1.56)$ & $6600(0.78)$ & -0.01 & $23300(0.87)$ & 0.36 & 0.55 \\
\hline & Hub-ensemble & $18300(1)$ & $21900(1)$ & $6140(1)$ & 3800 (1) & $8410(1)$ & -0.03 & $26800(1)$ & 0.43 & 0.64 \\
\hline & Renewal & $25600(1.4)$ & $33800(1.54)$ & $5420(0.88)$ & $5920(1.56)$ & $14200(1.69)$ & 0.17 & $34600(1.29)$ & 0.43 & 0.67 \\
\hline \multicolumn{11}{|l|}{ Deaths } \\
\hline \multirow{4}{*}{1 wk ahead } & Convolution & $255(1.03)$ & $343(1.01)$ & $82(0.89)$ & $142(1.23)$ & $31.1(0.75)$ & -0.18 & $399(1.19)$ & 0.42 & 0.79 \\
\hline & Crowd forecast & 265 (1.07) & $317(0.94)$ & $78.2(0.85)$ & $82(0.71)$ & $105(2.52)$ & 0.08 & $402(1.2)$ & 0.38 & 0.79 \\
\hline & Hub-ensemble & $248(1)$ & $338(1)$ & $92.2(1)$ & $115(1)$ & $41.6(1)$ & -0.04 & $334(1)$ & 0.62 & 0.92 \\
\hline & Renewal & $298(1.2)$ & $403(1.19)$ & $87(0.94)$ & $107(0.93)$ & $105(2.52)$ & -0.07 & $413(1.24)$ & 0.50 & 0.79 \\
\hline \multirow{4}{*}{2 wk ahead } & Convolution & $357(1.22)$ & $573(1.49)$ & $104(0.79)$ & $204(1.89)$ & $48.8(0.94)$ & -0.10 & $565(1.32)$ & 0.33 & 0.79 \\
\hline & Crowd forecast & $368(1.26)$ & $442(1.15)$ & $107(0.81)$ & $102(0.94)$ & $160(3.08)$ & 0.14 & $576(1.34)$ & 0.38 & 0.75 \\
\hline & Hub-ensemble & $292(1)$ & 385 (1) & $132(1)$ & $108(1)$ & $51.9(1)$ & 0.01 & $429(1)$ & 0.62 & 0.96 \\
\hline & Renewal & $524(1.79)$ & $671(1.74)$ & $155(1.17)$ & $133(1.23)$ & $236(4.55)$ & -0.02 & $750(1.75)$ & 0.50 & 0.71 \\
\hline
\end{tabular}


Table 3: Scores for three and four week ahead forecasts (cut to three significant digits and rounded). Note that scores for cases (which include the whole period from October 12th 2020 until March 1st 2021) and deaths (which include only forecasts from the 21st of December 2020 on) are computed on different subsets. Numbers in brackets show the metrics relative to the Hub ensemble (i.e. the median ensemble of all other models submitted to the German and Polish Forecast Hub, excluding our contributions). WIS is the mean weighted interval score (lower values are better), WIS - sd is the standard deviation of all scores achieved by a model. Dispersion, over-prediction and under-prediction together sum up to the weighted interval score. Bias (between -1 and 1, 0 is ideal) represents the general average tendency of a model to over- or underpredict. 50\% and 90\%coverage are the percentage of observed values that fell within the $50 \%$ and $90 \%$ prediction intervals of a model.

\begin{tabular}{|c|c|c|c|c|c|c|c|c|c|c|}
\hline & Model & WIS & WIS - sd & dispersion & Underpred. & Overpred. & Bias & Abs. error & $50 \%$-Cov. & $90 \%$-Cov. \\
\hline \multicolumn{11}{|l|}{ Cases } \\
\hline \multirow{3}{*}{3 wk ahead } & Crowd forecast & $27000(0.81)$ & $26200(0.64)$ & $4750(0.52)$ & $11000(1.43)$ & $11200(0.67)$ & 0.02 & $39000(0.83)$ & 0.14 & 0.48 \\
\hline & Hub-ensemble & $33400(1)$ & $40700(1)$ & $9130(1)$ & $7690(1)$ & $16600(1)$ & -0.01 & $46900(1)$ & 0.29 & 0.62 \\
\hline & Renewal & $50600(1.51)$ & $70000(1.72)$ & $10800(1.18)$ & $7710(1)$ & $32100(1.93)$ & 0.13 & $68700(1.46)$ & 0.29 & 0.55 \\
\hline \multirow{3}{*}{4 wk ahead } & Crowd forecast & $39200(0.7)$ & $38600(0.52)$ & $5970(0.49)$ & $15600(1.26)$ & $17600(0.56)$ & 0.07 & $54800(0.74)$ & 0.05 & 0.38 \\
\hline & Hub-ensemble & $55900(1)$ & $73700(1)$ & $12200(1)$ & $12400(1)$ & $31300(1)$ & 0.01 & $74400(1)$ & 0.24 & 0.52 \\
\hline & Renewal & $91700(1.64)$ & $135000(1.83)$ & $19500(1.6)$ & $8990(0.72)$ & $63200(2.02)$ & 0.09 & $125000(1.68)$ & 0.31 & 0.48 \\
\hline \multicolumn{11}{|l|}{ Deaths } \\
\hline \multirow{4}{*}{3 wk ahead } & Convolution & $541(1.7)$ & $802(2.45)$ & $157(0.91)$ & $279(3.01)$ & $105(1.91)$ & -0.04 & $747(1.53)$ & 0.54 & 0.75 \\
\hline & Crowd forecast & $414(1.3)$ & $526(1.6)$ & $137(0.8)$ & $82(0.88)$ & $194(3.52)$ & 0.12 & $648(1.33)$ & 0.42 & 0.83 \\
\hline & Hub-ensemble & $319(1)$ & $328(1)$ & $172(1)$ & $92.7(1)$ & $55.1(1)$ & -0.03 & $488(1)$ & 0.54 & 0.96 \\
\hline & Renewal & $724(2.27)$ & $916(2.79)$ & $249(1.45)$ & $158(1.7)$ & $317(5.75)$ & -0.01 & $1040(2.13)$ & 0.46 & 0.83 \\
\hline \multirow{4}{*}{4 wk ahead } & Convolution & $763(1.8)$ & $932(2.1)$ & $268(1.26)$ & $331(2.63)$ & $164(1.91)$ & 0.01 & $985(1.46)$ & 0.54 & 0.75 \\
\hline & Crowd forecast & $498(1.17)$ & $633(1.43)$ & $168(0.79)$ & $83.6(0.66)$ & $246(2.87)$ & 0.14 & 756 (1.12) & 0.38 & 0.79 \\
\hline & Hub-ensemble & $424(1)$ & $443(1)$ & $212(1)$ & $126(1)$ & $85.7(1)$ & -0.06 & $675(1)$ & 0.58 & 0.92 \\
\hline & Renewal & $959(2.26)$ & $1210(2.73)$ & $337(1.59)$ & $200(1.59)$ & $421(4.91)$ & -0.05 & $1350(2)$ & 0.50 & 0.79 \\
\hline
\end{tabular}




\section{A.5 Aggregate performance by location}

\section{A.5.1 Performance across locations in absolute terms}

A

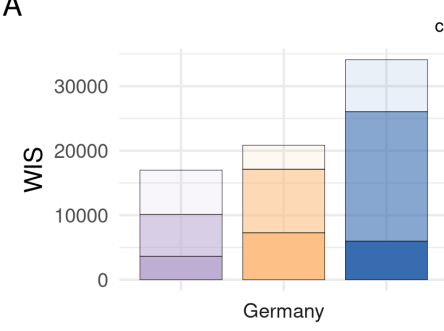

C

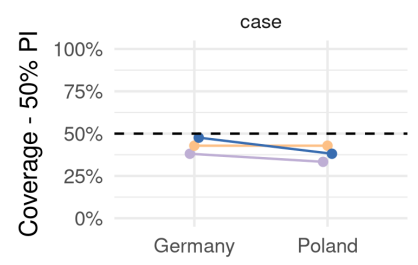

$\mathrm{E}$

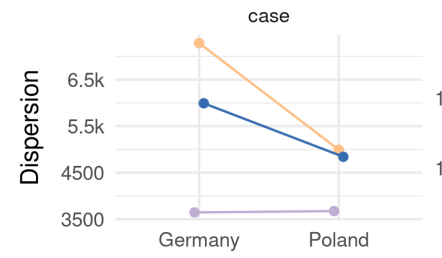

G

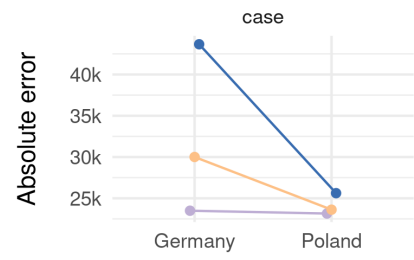

case
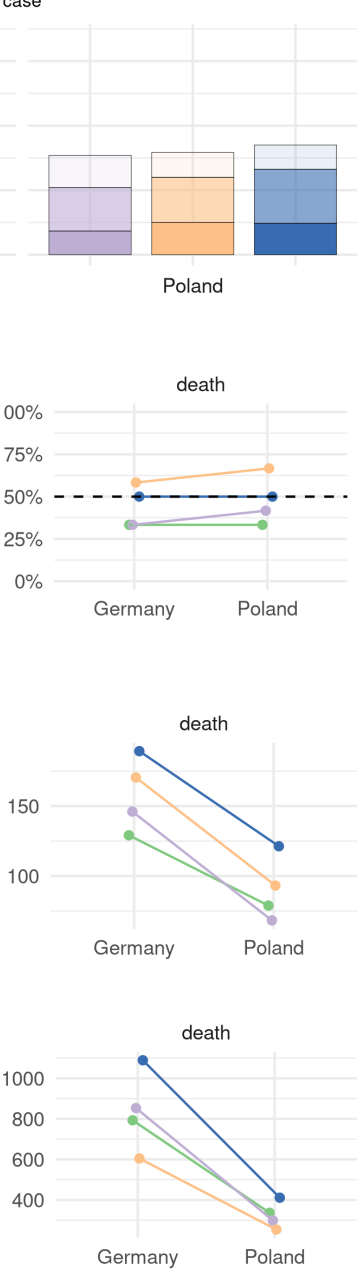

B

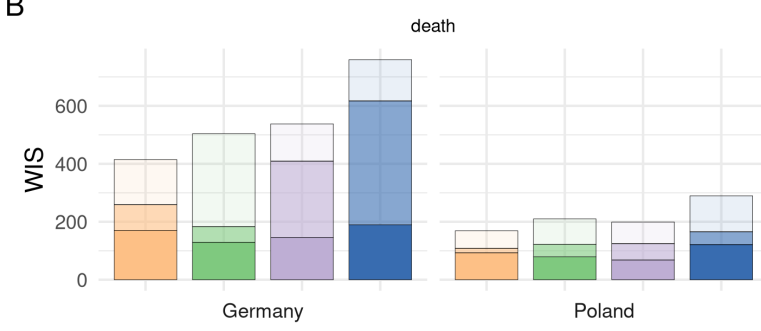

$\mathrm{D}$

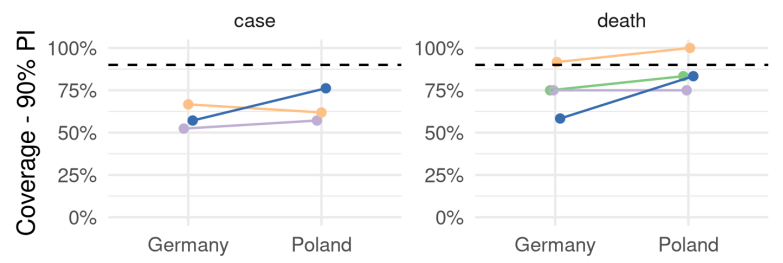

$\mathrm{F}$

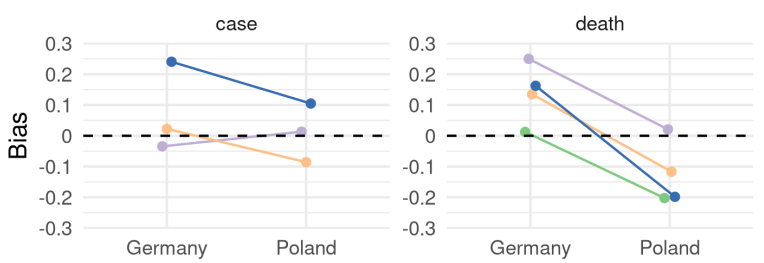

$\mathrm{H}$

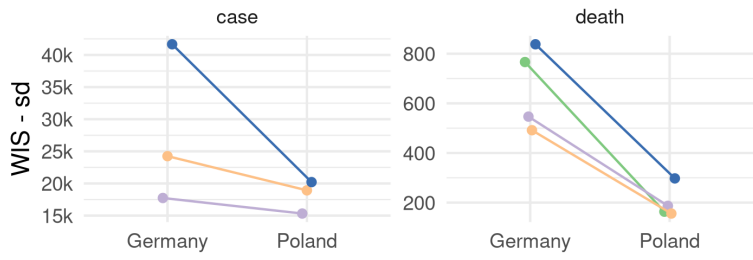

WIS components $\square$ Dispersion $\square$ Under-prediction $\square$ Over-prediction

Model $\square$ Convolution $\square$ Crowd forecast $\square$ Hub-ensemble $\square$ Renewal

Figure 6: Visualisation of aggregate performance metrics across locations. A: mean weighted interval score (WIS) across horizons. B: median WIS. C: Absolute error of the median forecast. D: Standard deviation of the WIS. E: Dispersion (higher values mean further spread out forecast). F: Bias, i.e. general tendency to over- or underpredict. Values are between -1 (complete under-prediction) and 1 (complete over-prediction) and 0 ideally. G: Empirical coverage of the 50\% prediction intervals. F: Empirical coverage of the $90 \%$ prediction intervals. 


\section{A.6 Performance across locations in relative terms}

A

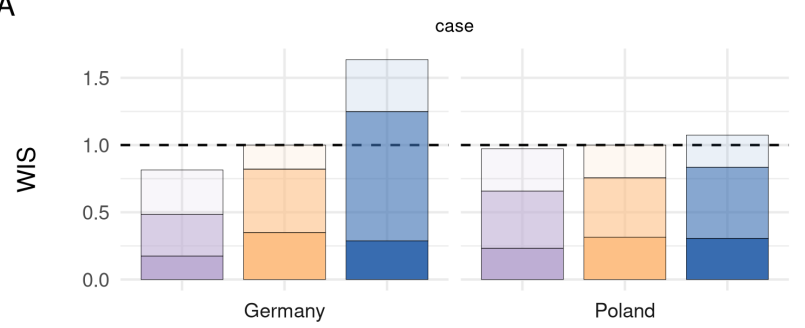

C

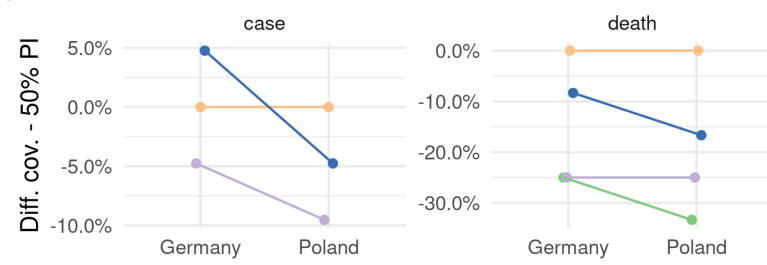

$\mathrm{E}$

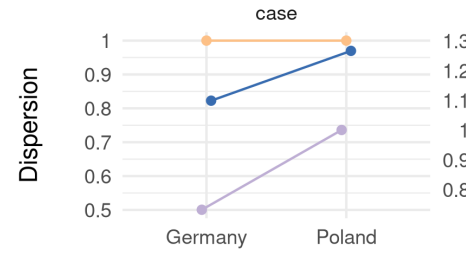

G
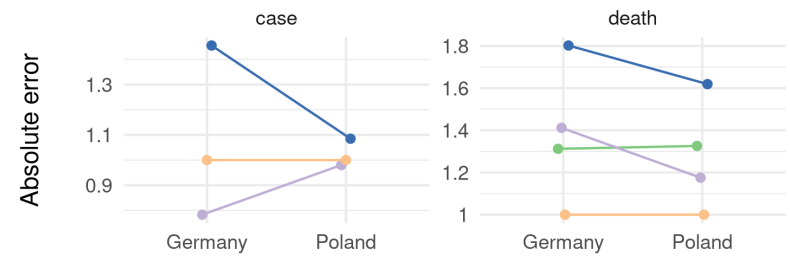

B

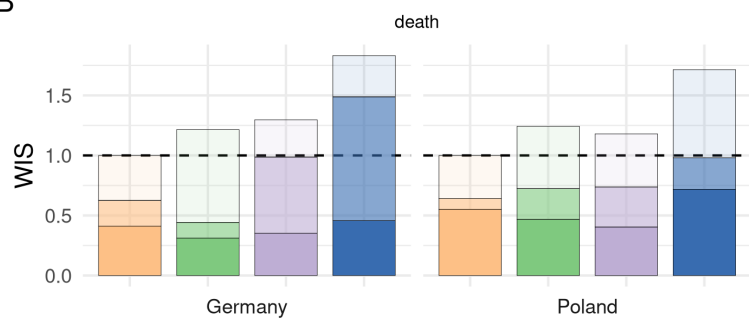

$\mathrm{D}$

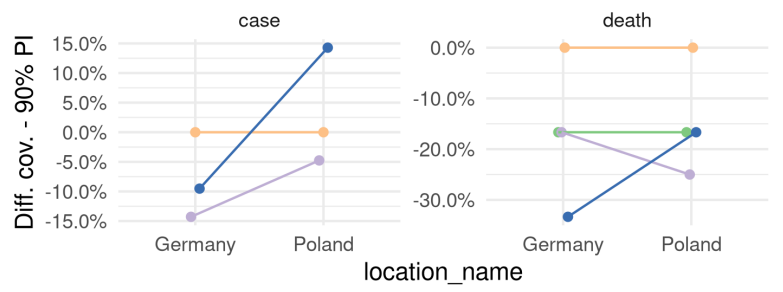

$\mathrm{F}$

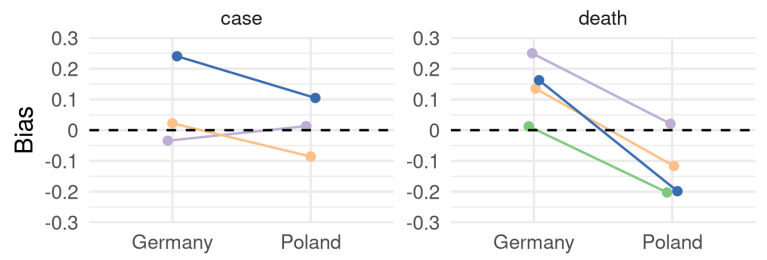

$\mathrm{H}$

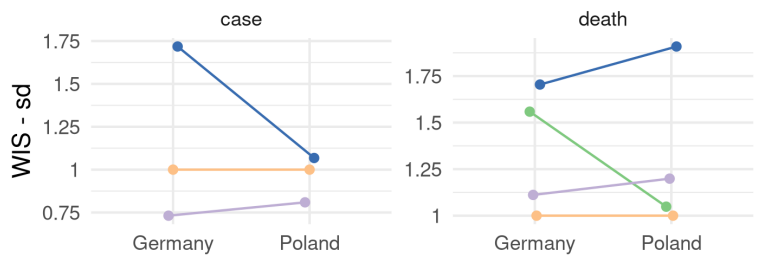

WIS components $\square$ Dispersion $\square$ Under-prediction $\square$ Over-prediction

Model $\square$ Convolution $\square$ Crowd forecast $\square$ Hub-ensemble $\square$ Renewal

Figure 7: Visualisation of aggregate performance metrics across locations relative to the Hub ensemble (excluding our contributions). A: mean weighted interval score (WIS) across horizons. B: median WIS. C: Absolute error of the median forecast. D: Standard deviation of the WIS. E: Dispersion (higher values mean further spread out forecast). F: Bias, i.e. general tendency to over- or underpredict. Values are between -1 (complete under-prediction) and 1 (complete over-prediction) and 0 ideally. G: Empirical coverage of the 50\% prediction intervals. F: Empirical coverage of the $90 \%$ prediction intervals. 


\section{A.7 Visualisation of daily reported cases and deaths}
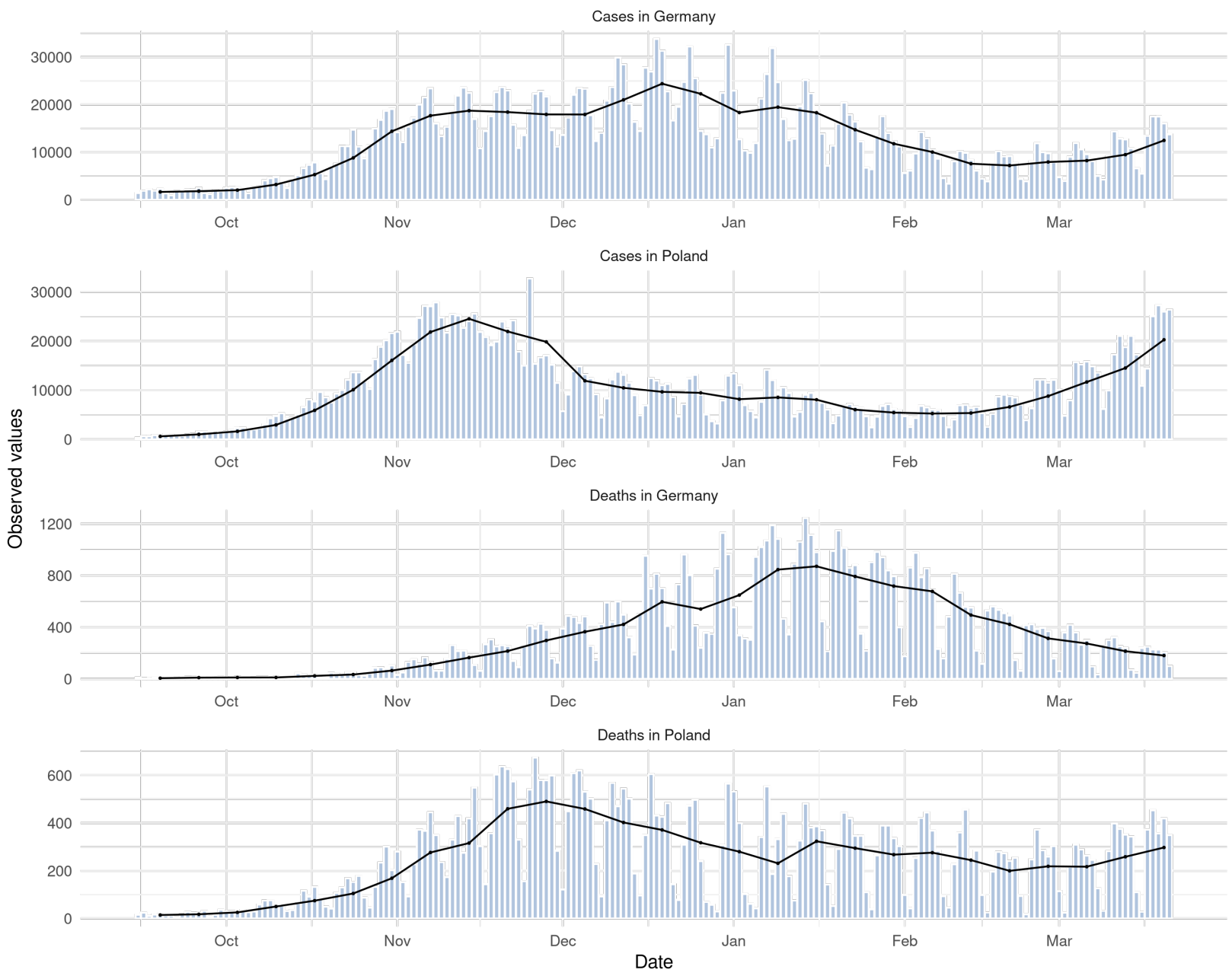

Figure 8: Visualisation of daily report data. The black line represents weekly data divided by seven. Data were last accessed through the German and Polish Forecast Hub on August 212021. 


\section{A.8 Visualisation of scores and forecasts 1, 3, 4 weeks ahead}

A

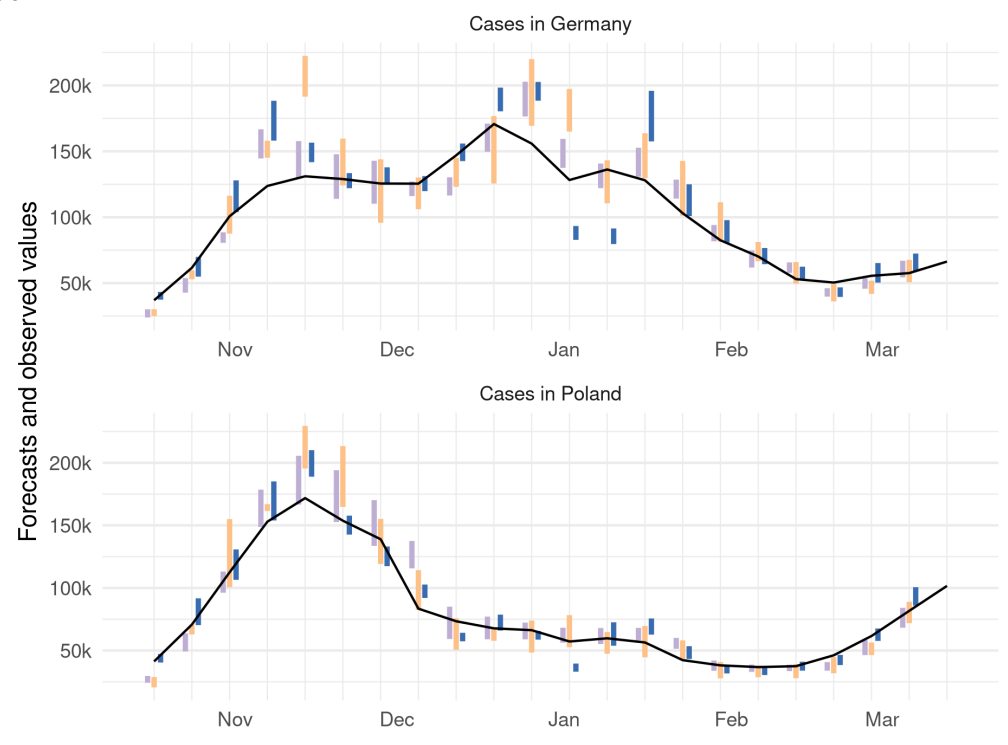

C

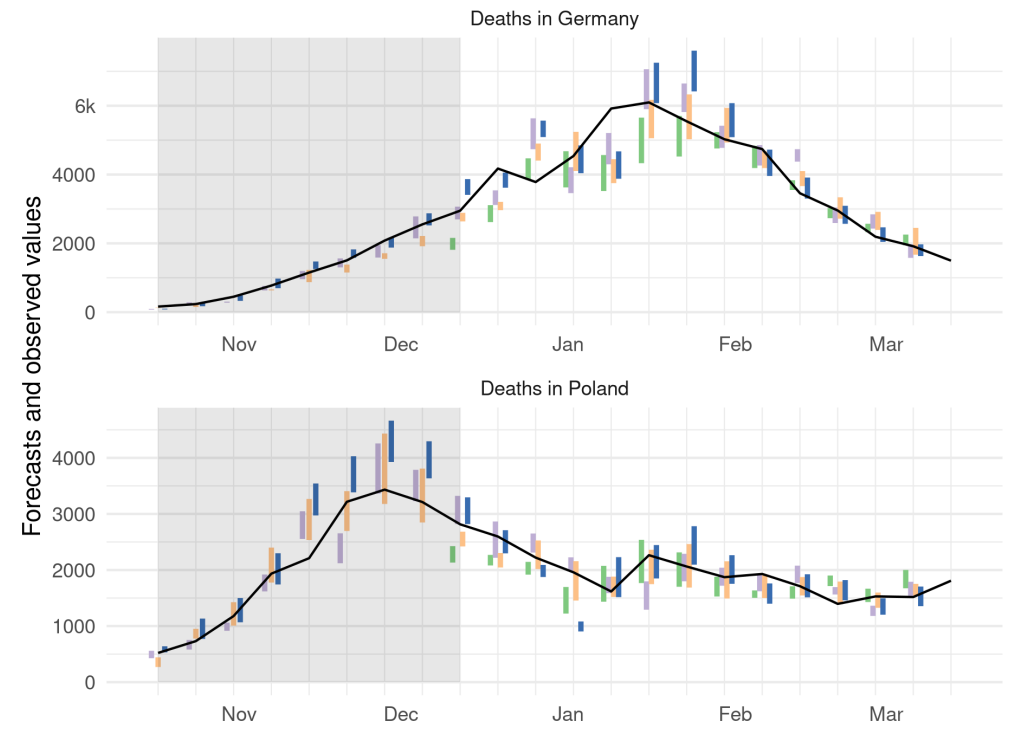

B

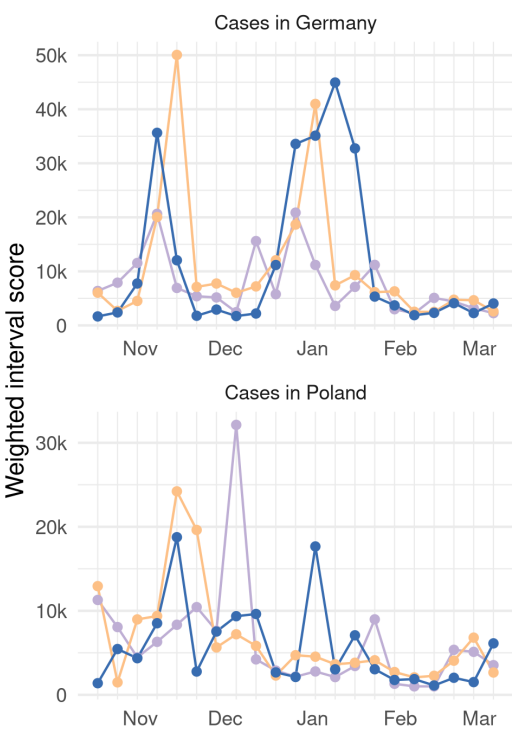

D

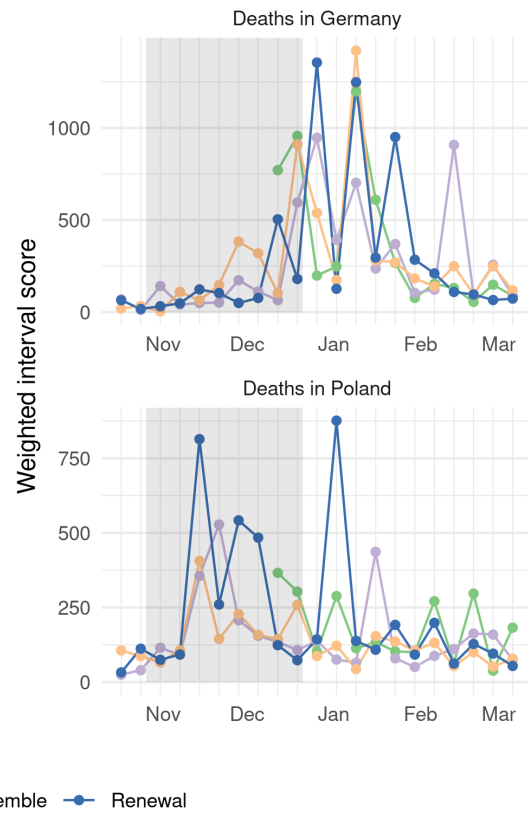

Figure 9: A, C: Visualisation of 50\% prediction intervals of one week ahead forecasts against the reported values. Forecasts that were not scored (because there was no complete set of death forecasts available) are greyed out. B, D: Visualisation of corresponding WIS. 
A

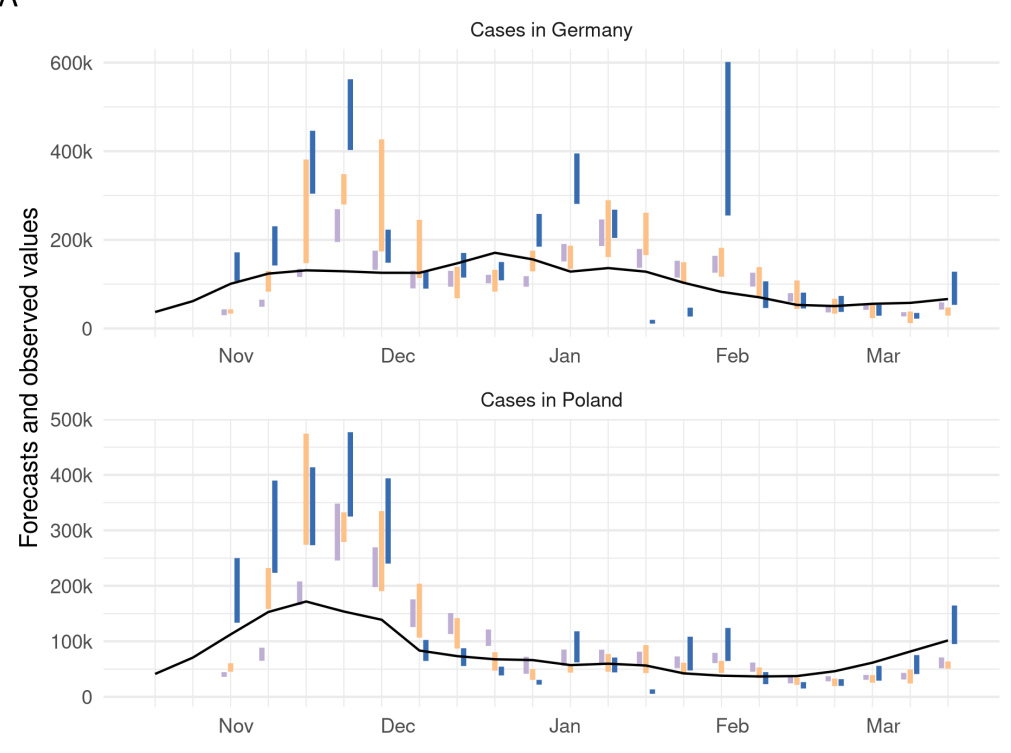

C

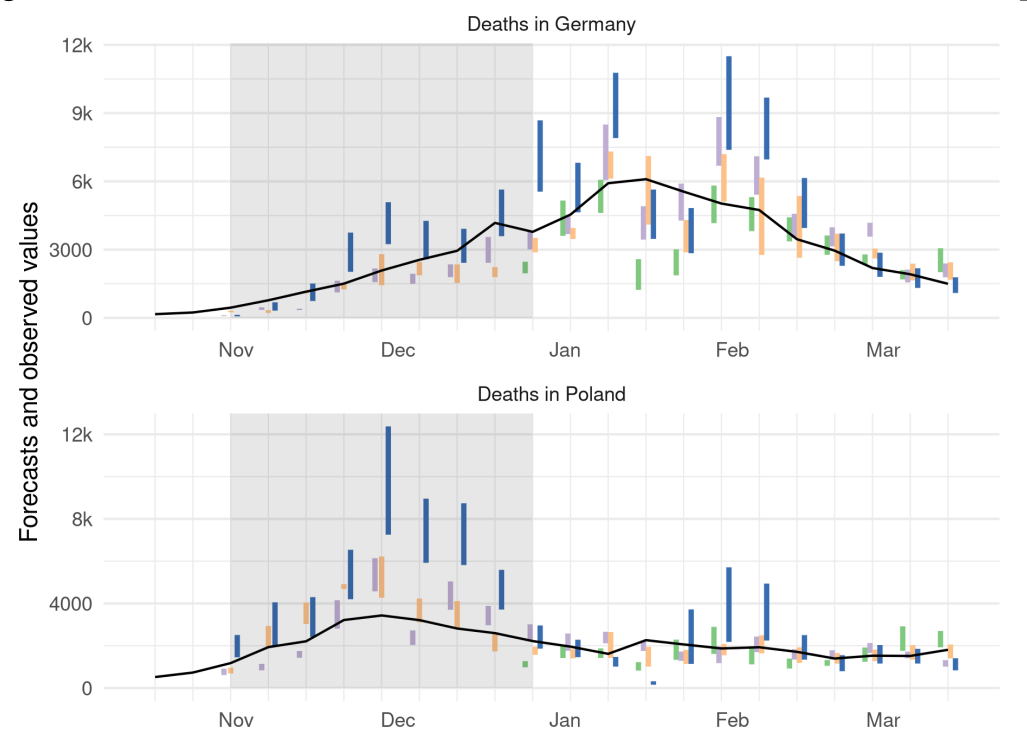

B

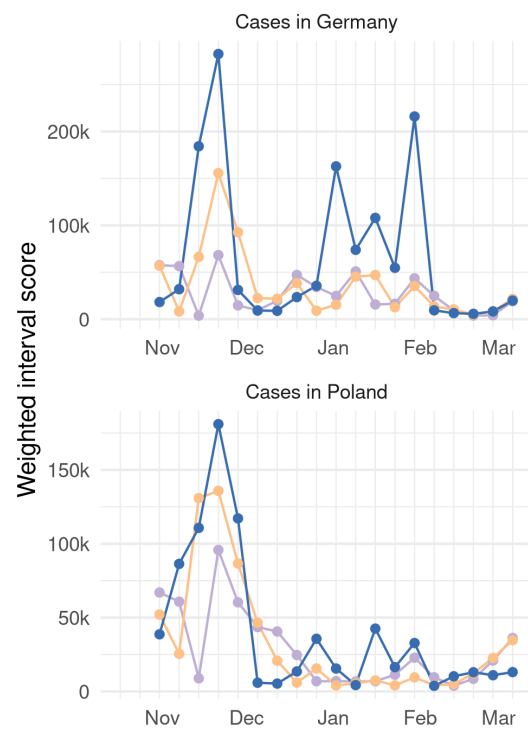

D

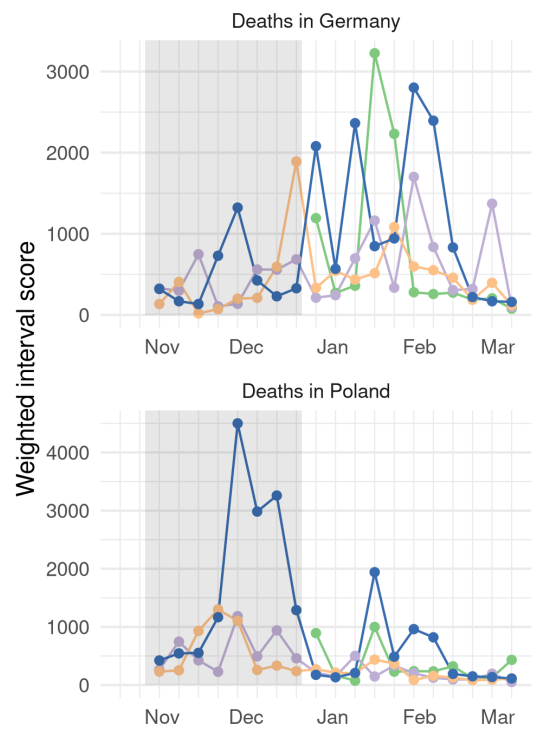

Model $\rightarrow-$ Convolution $\rightarrow$ Crowd forecast $\rightarrow$ Hub-ensemble $\rightarrow$ Renewal

Figure 10: A, C: Visualisation of $50 \%$ prediction intervals of three week ahead forecasts against the reported values. Forecasts that were not scored (because there was no complete set of death forecasts available) are greyed out. B, D: Visualisation of corresponding WIS. 
A

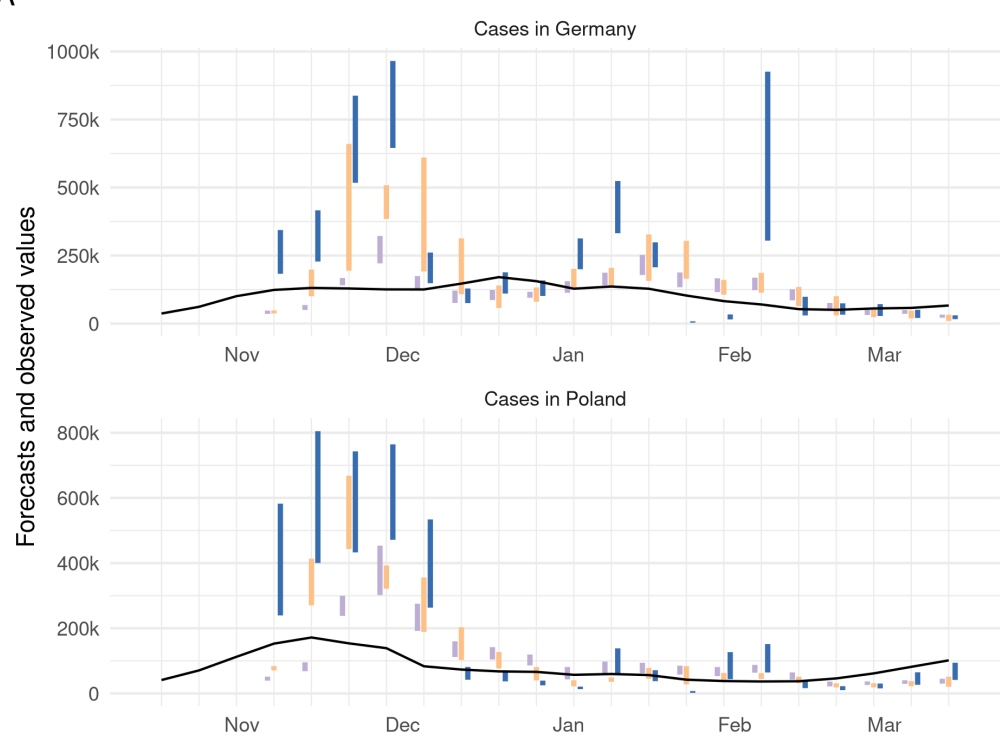

C

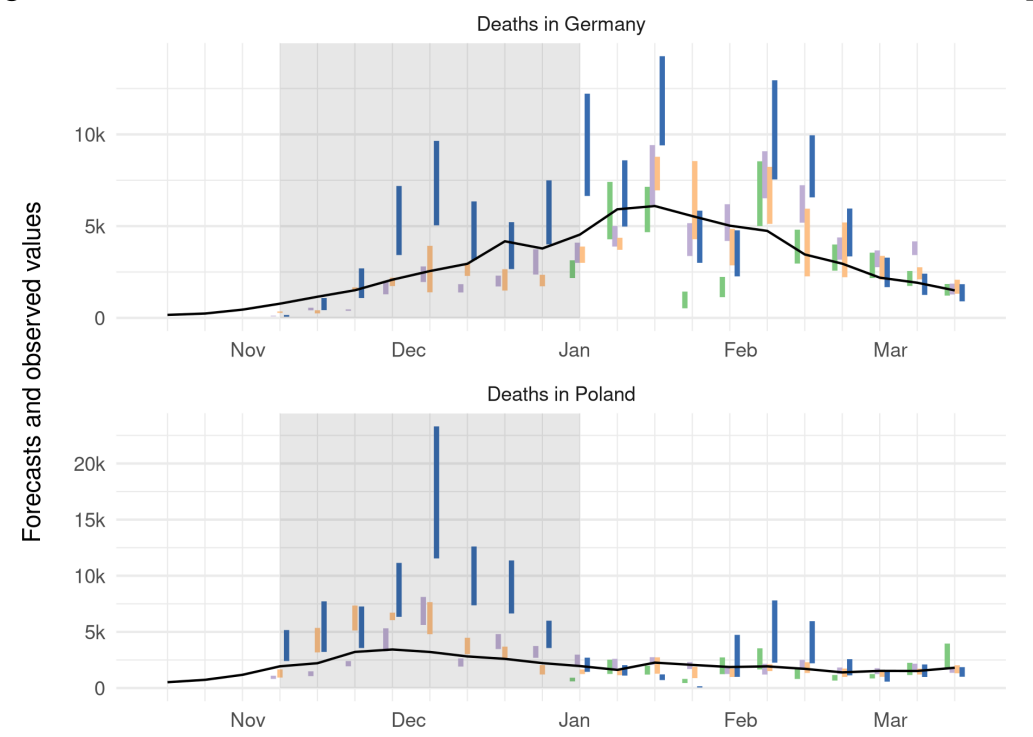

B

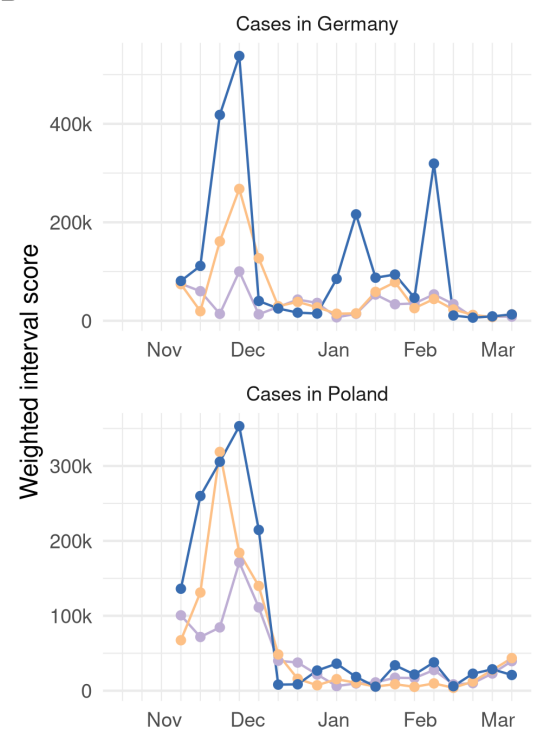

D

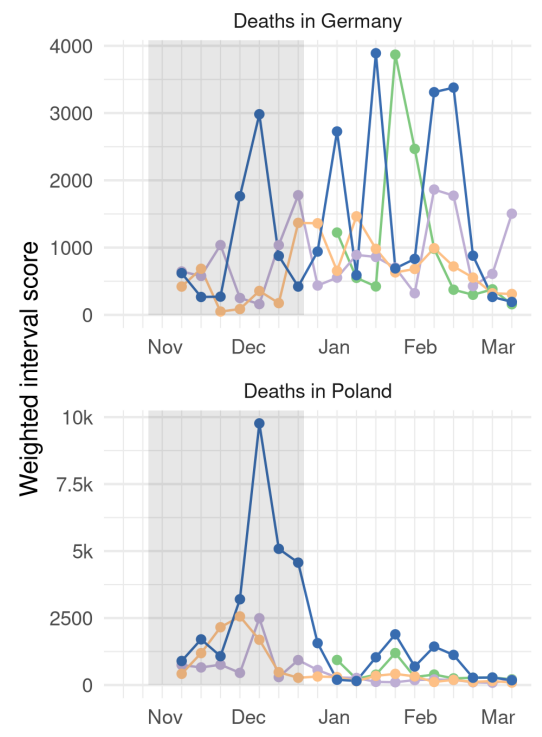

Model $\rightarrow$ Convolution $\rightarrow$ Crowd forecast $\rightarrow$ Hub-ensemble $\rightarrow$ Renewal

Figure 11: A, C: Visualisation of $50 \%$ prediction intervals of four week ahead forecasts against the reported values. Forecasts that were not scored (because there was no complete set of death forecasts available) are greyed out. B, D: Visualisation of corresponding WIS. 


\section{A.9 Distribution of scores}

\section{A.9.1 Absolute scores}

A
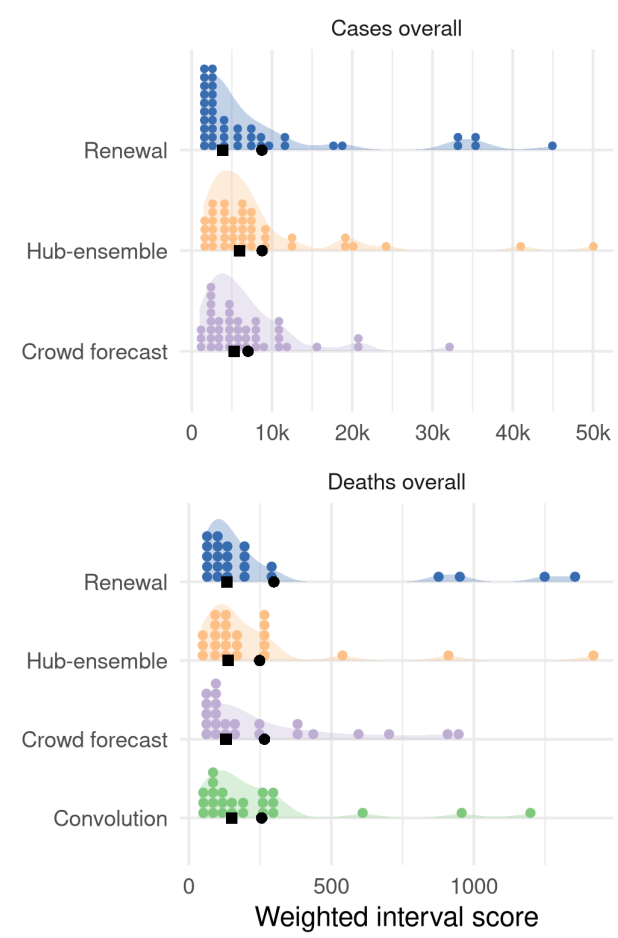

B
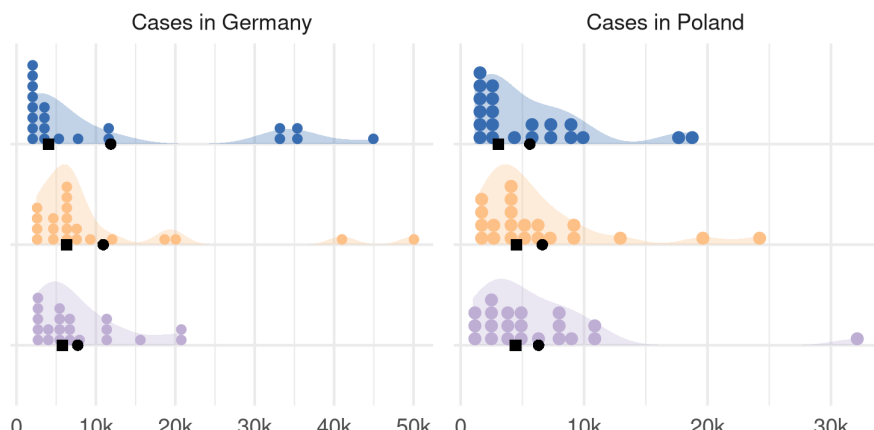

Deaths in Germany

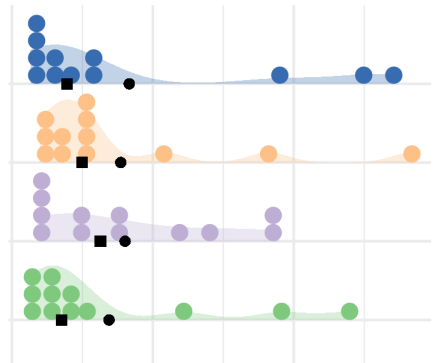

$500 \quad 1000$
Deaths in Poland

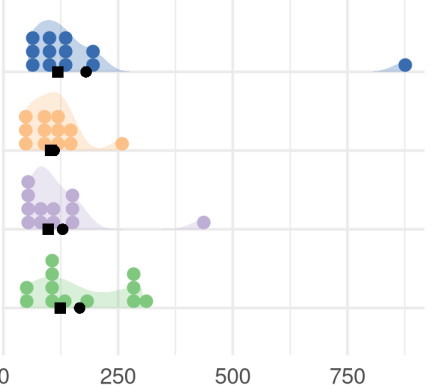

$$
\text { Model Convolution } \odot \text { Crowd forecast } \odot \text { Hub-ensemble } \odot \text { Renewal }
$$

Figure 12: A: Distribution of weighted interval scores for one week ahead forecasts of the different models and forecast targets. B: Distribution of WIS separate by country. 
A
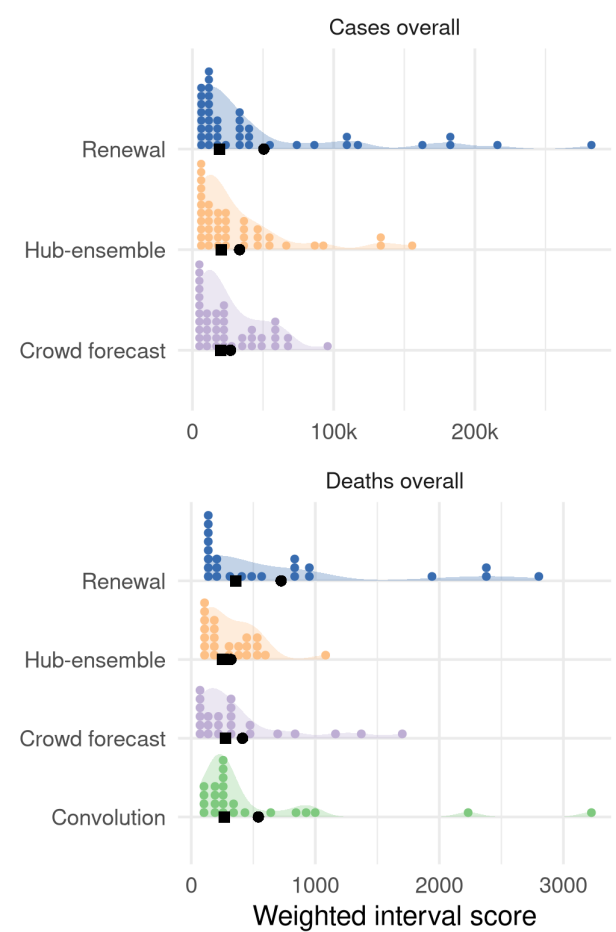

Model Convolution

B
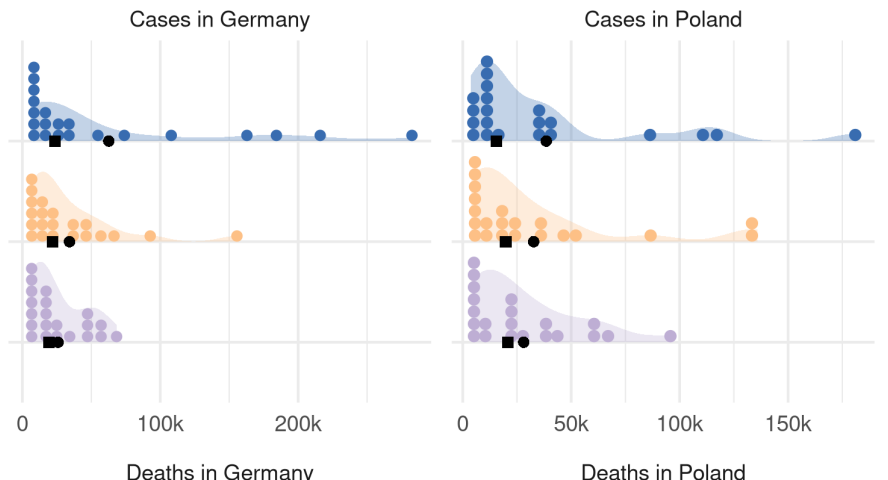

Deaths in Germany

Deaths in Poland

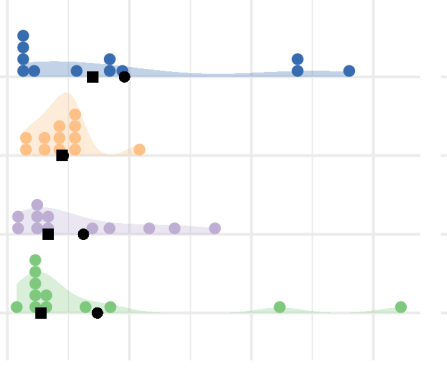

1000

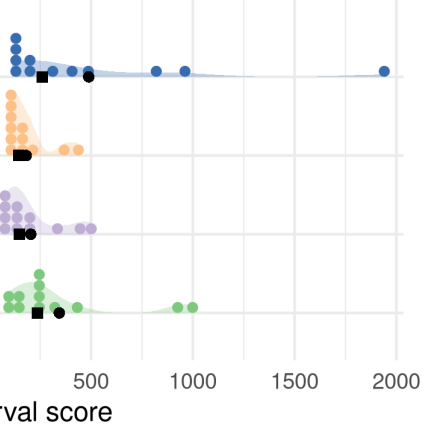

$\begin{array}{ccr}3000 & 0 & 500 \\ \text { Weighted interval score }\end{array}$

Renewal

Figure 13: A: Distribution of weighted interval scores for three week ahead forecasts of the different models and forecast targets. B: Distribution of WIS separate by country. 
A
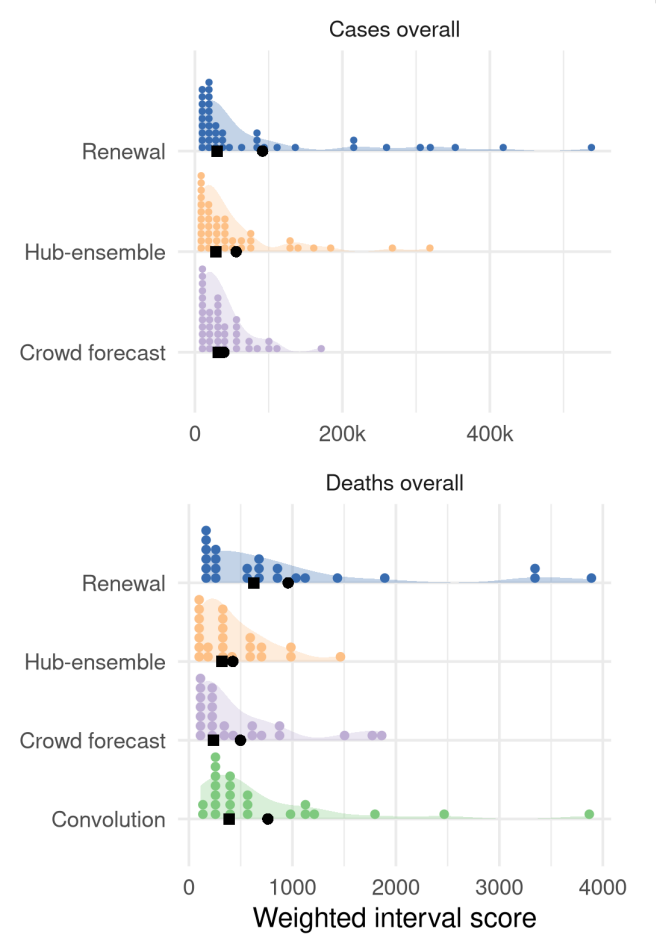

Model Convolution
B
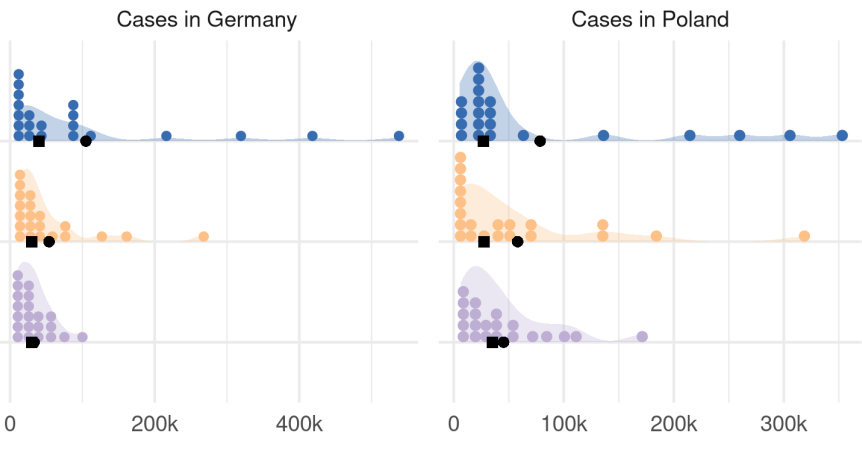

Deaths in Germany

Deaths in Poland

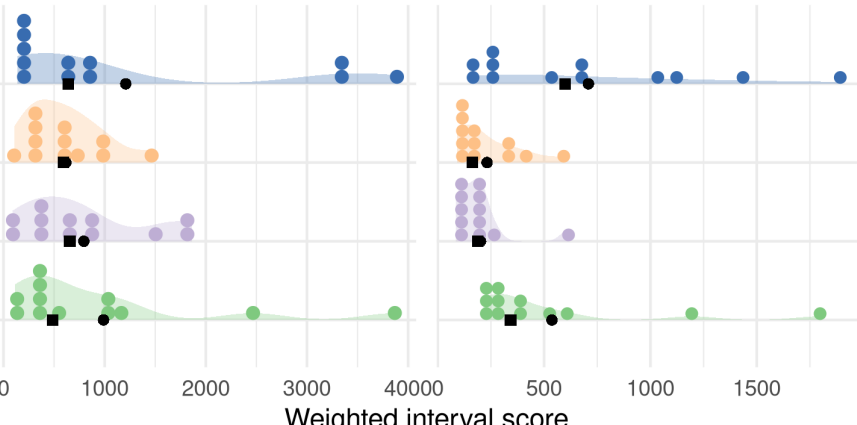

Crowd forecast Hub-ensemble

Renewal

Figure 14: A: Distribution of weighted interval scores for four week ahead forecasts of the different models and forecast targets. B: Distribution of WIS separate by country. 


\section{A.9.2 Ranks achieved by forecasts}

A
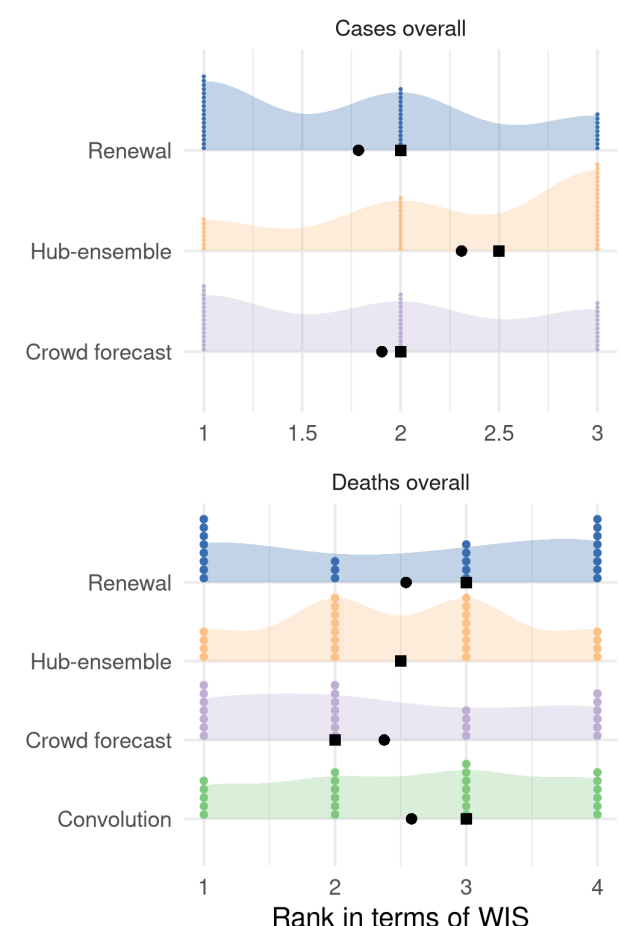

Model Convolution

B
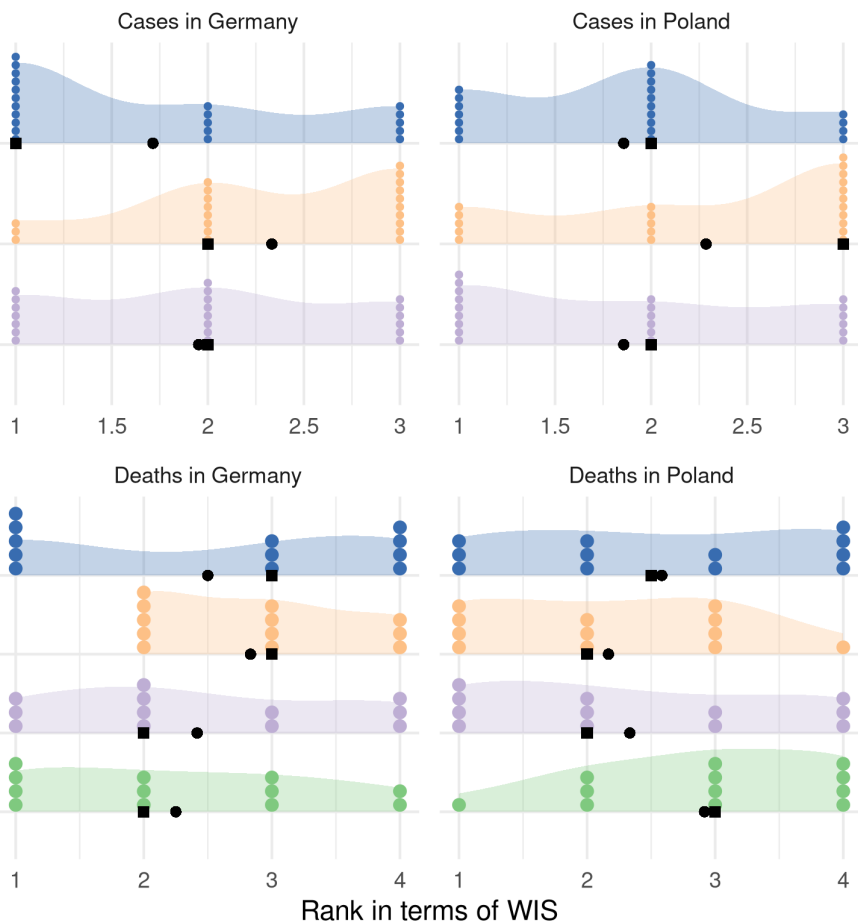

Rank in terms of WIS

Figure 15: A: Distribution of the ranks (determined by the weighted interval score) for one week ahead forecasts of the different models and forecast targets. B: Distribution of ranks separate by country. 
A
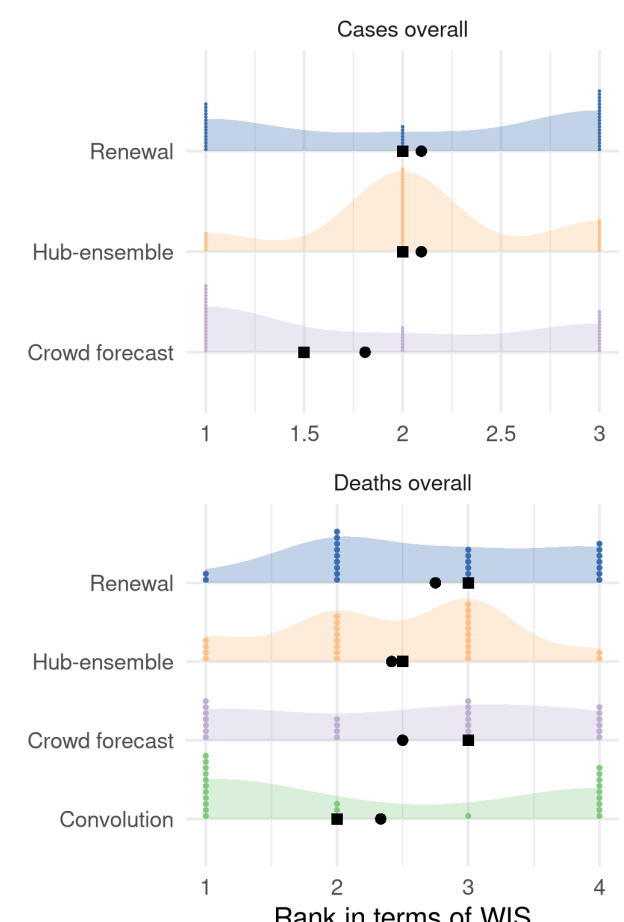

Model Convolution

B
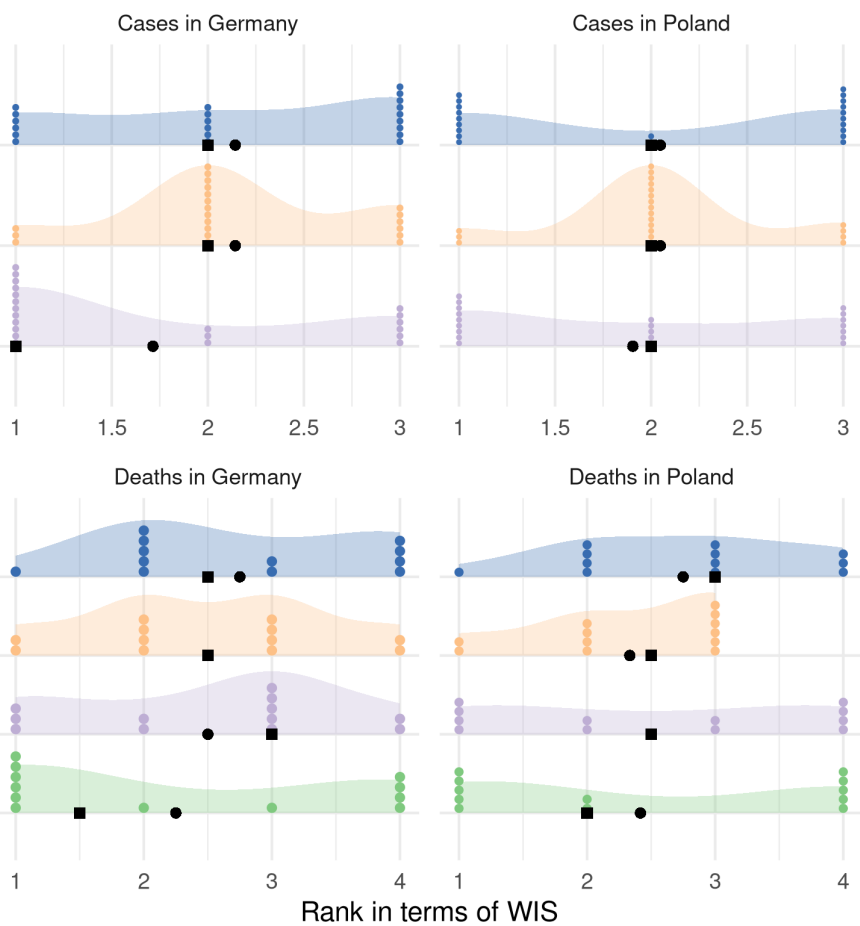

Rank in terms of WIS

Crowd forecast Hub-ensemble Renewal

Figure 16: A: Distribution of the ranks (determined by the weighted interval score) for two week ahead forecasts of the different models and forecast targets. B: Distribution of ranks separate by country. 
A
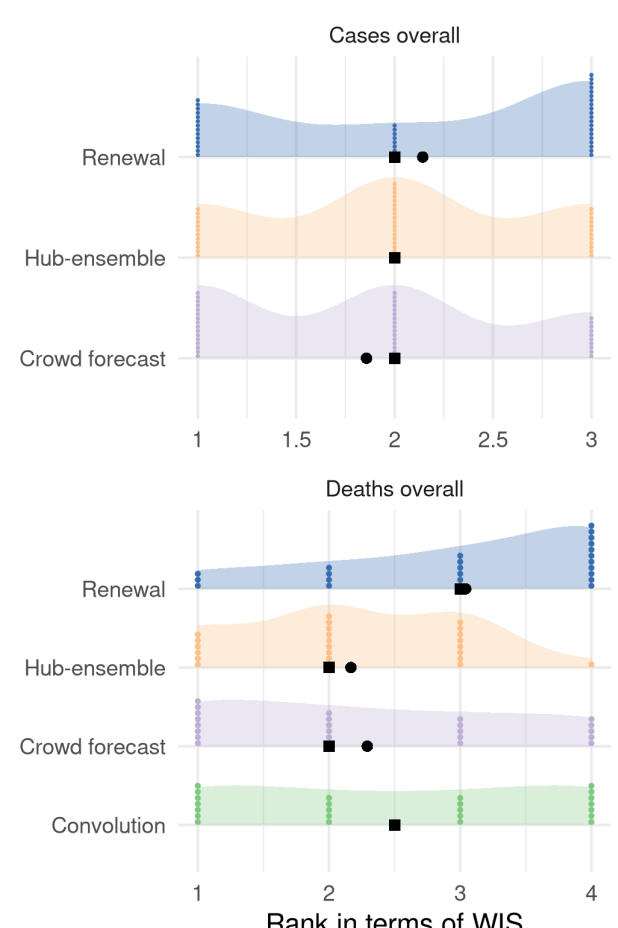

Rank in terms of WIS
B
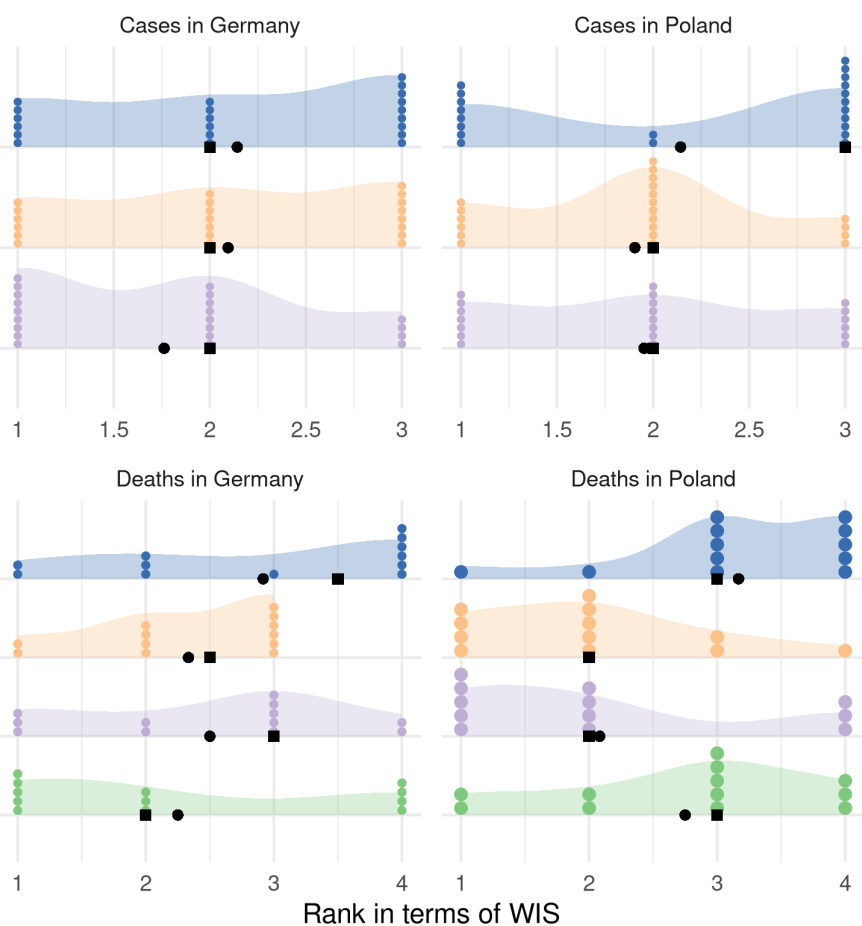

Model Convolution

Figure 17: A: Distribution of the ranks (determined by the weighted interval score) for three week ahead forecasts of the different models and forecast targets. B: Distribution of ranks separate by country. 
A
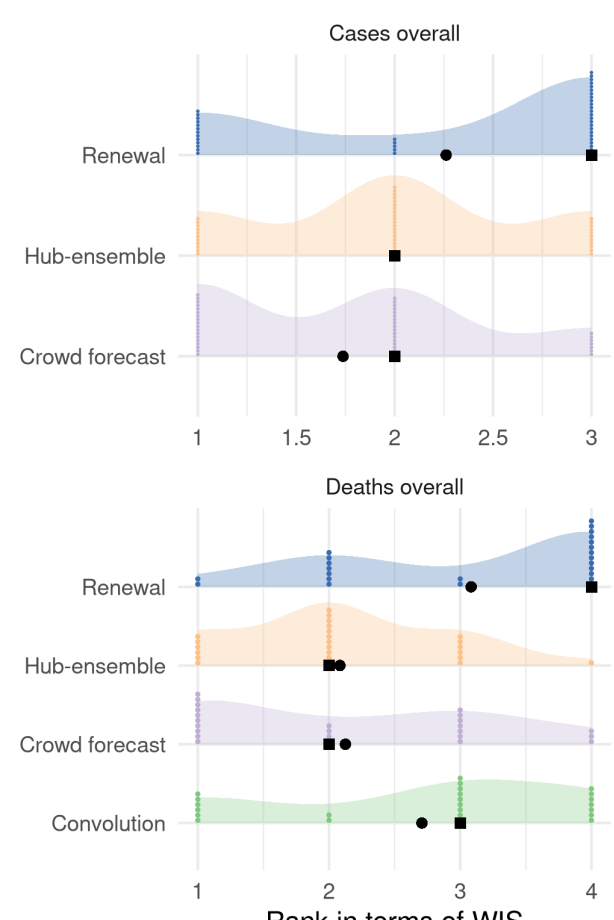

Rank in terms of WIS
B
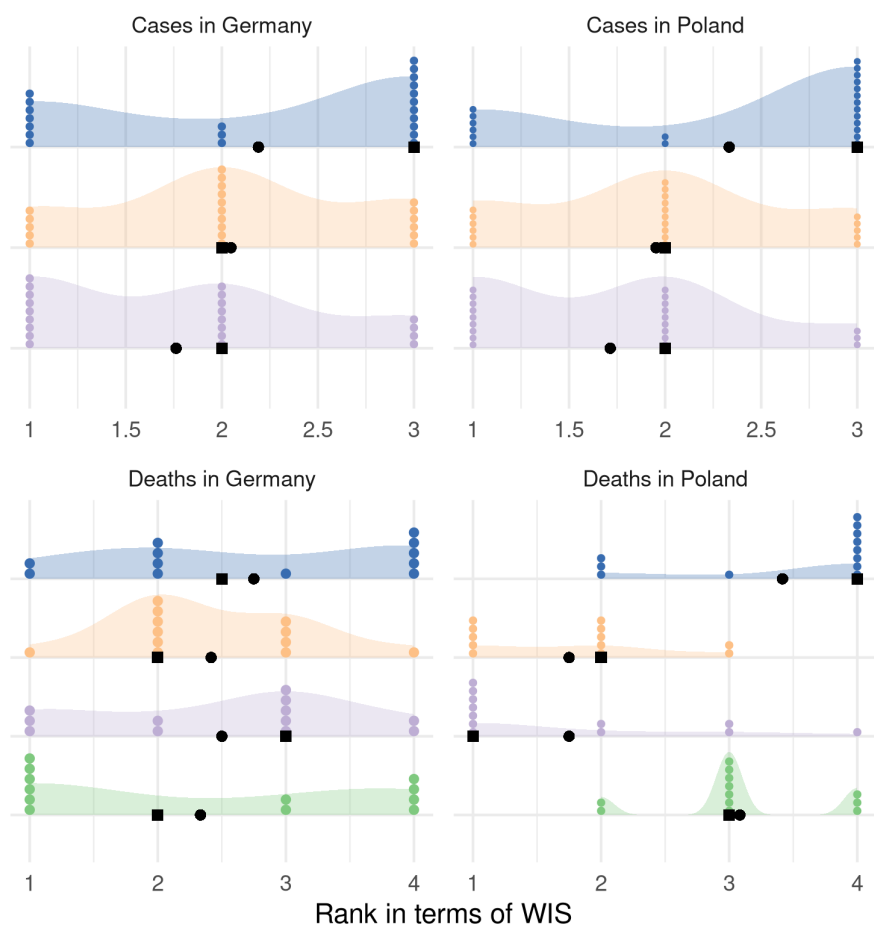

Model Convolution $\odot$ Crowd forecast $\odot$ Hub-ensemble $\bigcirc$ Renewal

Figure 18: A: Distribution of the ranks (determined by the weighted interval score) for four week ahead forecasts of the different models and forecast targets. B: Distribution of ranks separate by country. 


\section{A.10 Comparison of ensembles}

\section{A.10.1 Performance visualisation mean ensemble}

A

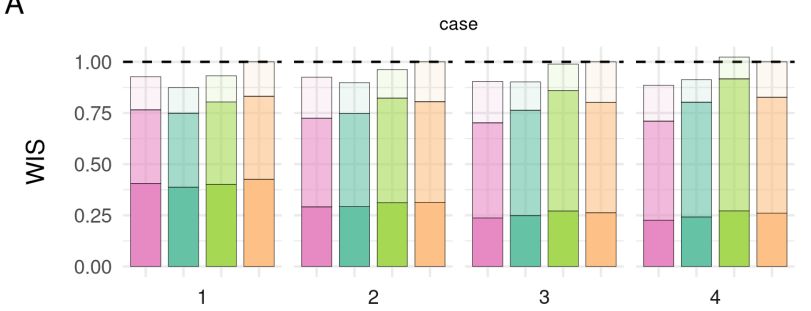

$\mathrm{C}$

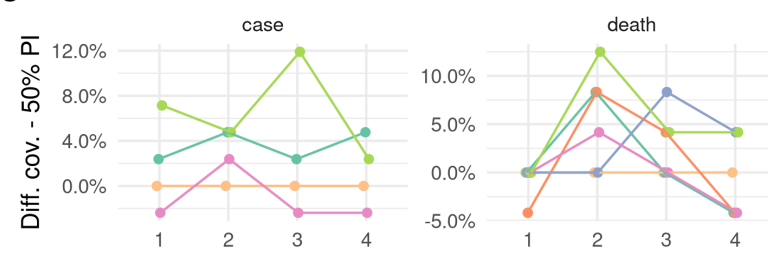

E

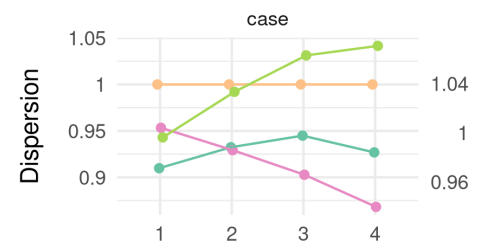

G

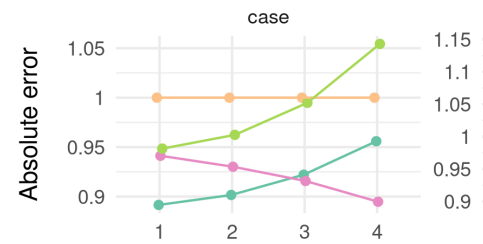

B

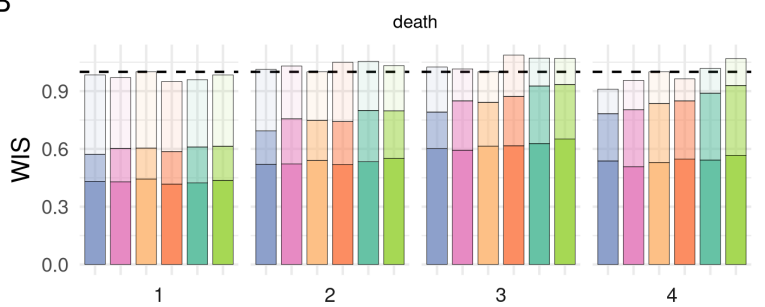

$\mathrm{D}$
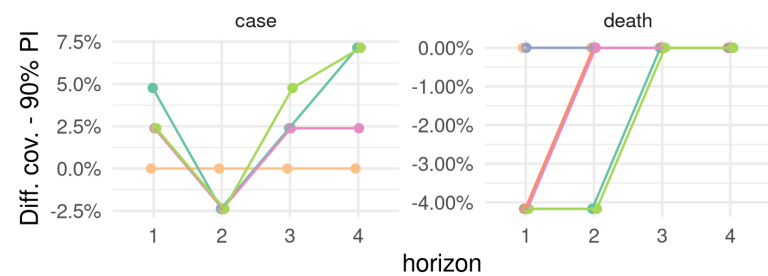

$\mathrm{F}$

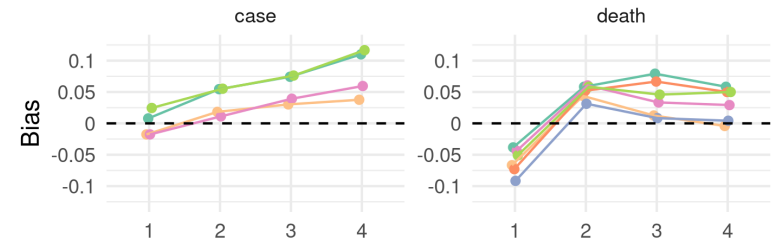

$\mathrm{H}$

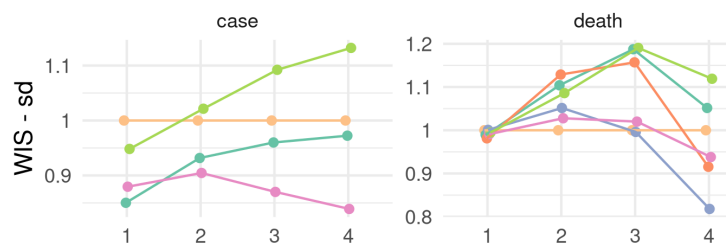

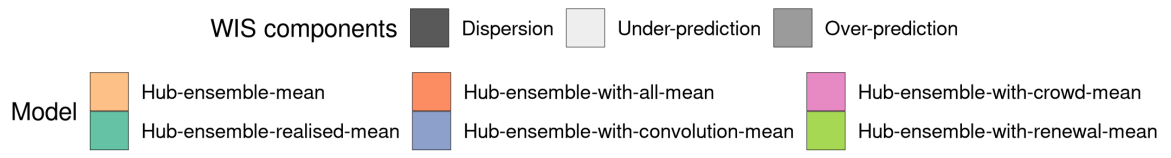

Figure 19: Visualisation of aggregate performance metrics across forecast horizons for the different versions of the Hub mean ensemble. "Hub-ensemble" excludes all our models, Hub-ensemble-all includes all of our models, "Hub-ensemble-real" is the real hub-ensemble with the renewal model and the crowd forecasts included. Values (except for Bias) are computed as differences to the Hub ensemble excluding our contributions. For Coverage, this is an absolute difference, for other metrics this is a percentage difference. A: mean weighted interval score (WIS) across horizons. B: median WIS. C: Absolute error of the median forecast. D: Standard deviation of the WIS. E: Dispersion (higher values mean greater spread of the forecast). F: Bias, i.e. general tendency to over- or underpredict. Values are between -1 (complete under-prediction) and 1 (complete over-prediction) and 0 ideally. G: Empirical coverage of the 50\% prediction intervals. F: Empirical coverage of the $90 \%$ prediction intervals

\section{A.10.2 Tables median ensemble}


Table 4: Scores for one and two week ahead forecasts (cut to three significant digits and rounded) for the different versions of the median ensemble. Note that scores for cases (which include the whole period from October 12th 2020 until March 1st 2021) and deaths (which include only forecasts from the 21st of December 2020 on) are computed on different subsets. Numbers in brackets show the metrics relative to the Hub ensemble (i.e. the median ensemble of all other models submitted to the German and Polish Forecast Hub, excluding our contributions). WIS is the mean weighted interval score (lower values are better), WIS - sd is the standard deviation of all scores achieved by a model. Dispersion, over-prediction and under-prediction together sum up to the weighted interval score. Bias (between -1 and 1,0 is ideal) represents the general average tendency of a model to over- or underpredict. $50 \%$ and $90 \%$ coverage are the percentage of observed values that fell within the $50 \%$ and $90 \%$ prediction intervals of a model.

\begin{tabular}{|c|c|c|c|c|c|c|c|c|c|c|}
\hline & Model & WIS & WIS - sd & dispersion & Underpred. & Overpred. & Bias & Abs. error & $50 \%$-Cov. & $90 \%$-Cov \\
\hline \multicolumn{11}{|l|}{ Cases } \\
\hline & Hub-ensemble & $8770(1)$ & $11700(1)$ & $3670(1)$ & $1230(1)$ & $3870(1)$ & -0.04 & $12700(1)$ & 0.57 & 0.81 \\
\hline \multirow{3}{*}{1 wk ahead } & Hub-ensemble-realised & $6970(0.79)$ & $8260(0.71)$ & $3060(0.83)$ & $943(0.77)$ & $2970(0.77)$ & 0.04 & $10800(0.85)$ & 0.55 & 0.83 \\
\hline & Hub-ensemble-with-crowd & $7820(0.89)$ & $9630(0.82)$ & $3270(0.89)$ & $1210(0.98)$ & $3330(0.86)$ & -0.02 & $12000(0.94)$ & 0.48 & 0.81 \\
\hline & Hub-ensemble-with-renewal & $7960(0.91)$ & $10300(0.88)$ & $3190(0.87)$ & $1020(0.83)$ & $3760(0.97)$ & 0.04 & $12100(0.95)$ & 0.57 & 0.83 \\
\hline \multirow{4}{*}{2 wk ahead } & Hub-ensemble & $18300(1)$ & $21900(1)$ & $6140(1)$ & $3800(1)$ & $8410(1)$ & -0.03 & $26800(1)$ & 0.43 & 0.64 \\
\hline & Hub-ensemble-realised & $16400(0.9)$ & $19600(0.89)$ & $5350(0.87)$ & $3290(0.87)$ & $7730(0.92)$ & 0.02 & $24200(0.9)$ & 0.43 & 0.69 \\
\hline & Hub-ensemble-with-crowd & $16900(0.92)$ & $19600(0.89)$ & $5230(0.85)$ & $4310(1.13)$ & $7370(0.88)$ & 0.00 & $24600(0.92)$ & 0.38 & 0.64 \\
\hline & Hub-ensemble-with-renewal & $17500(0.96)$ & $21400(0.98)$ & $5830(0.95)$ & $2880(0.76)$ & $8770(1.04)$ & 0.00 & $25500(0.95)$ & 0.45 & 0.71 \\
\hline \multicolumn{11}{|l|}{ Deaths } \\
\hline \multirow{6}{*}{1 wk ahead } & Hub-ensemble & $248(1)$ & $338(1)$ & $92.2(1)$ & $115(1)$ & $41.6(1)$ & -0.04 & $334(1)$ & 0.62 & 0.92 \\
\hline & Hub-ensemble-realised & $235(0.95)$ & $332(0.98)$ & $88.6(0.96)$ & $90.4(0.79)$ & $55.5(1.33)$ & -0.01 & $323(0.97)$ & 0.62 & 0.88 \\
\hline & Hub-ensemble-with-all & $234(0.94)$ & $331(0.98)$ & $85.2(0.92)$ & $98.1(0.85)$ & $50.2(1.21)$ & -0.05 & $329(0.99)$ & 0.62 & 0.92 \\
\hline & Hub-ensemble-with-convolution & $234(0.94)$ & $329(0.97)$ & $90.7(0.98)$ & $118(1.03)$ & $25.3(0.61)$ & -0.08 & $333(1)$ & 0.62 & 0.92 \\
\hline & Hub-ensemble-with-crowd & $239(0.96)$ & $337(1)$ & $85.2(0.92)$ & $99.6(0.87)$ & $54.2(1.3)$ & -0.03 & $322(0.96)$ & 0.62 & 0.92 \\
\hline & Hub-ensemble-with-renewal & $246(0.99)$ & $342(1.01)$ & $91.5(0.99)$ & $106(0.92)$ & $48.6(1.17)$ & -0.06 & $342(1.02)$ & 0.67 & 0.92 \\
\hline \multirow{6}{*}{2 wk ahead } & Hub-ensemble & $292(1)$ & $385(1)$ & $132(1)$ & $108(1)$ & $51.9(1)$ & 0.01 & $429(1)$ & 0.62 & 0.96 \\
\hline & Hub-ensemble-realised & $296(1.01)$ & $398(1.03)$ & $125(0.95)$ & $91(0.84)$ & $80.2(1.55)$ & 0.05 & $486(1.13)$ & 0.58 & 0.92 \\
\hline & Hub-ensemble-with-all & $303(1.04)$ & $423(1.1)$ & $115(0.87)$ & $122(1.13)$ & $66.1(1.27)$ & 0.00 & $483(1.13)$ & 0.62 & 0.88 \\
\hline & Hub-ensemble-with-convolution & $270(0.92)$ & $385(1)$ & $121(0.92)$ & $119(1.1)$ & $29.9(0.58)$ & -0.04 & $403(0.94)$ & 0.58 & 0.96 \\
\hline & Hub-ensemble-with-crowd & $303(1.04)$ & $392(1.02)$ & $122(0.92)$ & $106(0.98)$ & $74.6(1.44)$ & 0.03 & $499(1.16)$ & 0.58 & 0.92 \\
\hline & Hub-ensemble-with-renewal & $296(1.01)$ & $397(1.03)$ & $128(0.97)$ & $97.1(0.9)$ & $71.2(1.37)$ & -0.01 & $462(1.08)$ & 0.67 & 0.92 \\
\hline
\end{tabular}

\section{A.10.3 Tables mean ensemble}


Table 5: Scores for three and four week ahead forecasts (cut to three significant digits and rounded) for the different versions of the median ensemble. Note that scores for cases (which include the whole period from October 12th 2020 until March 1st 2021) and deaths (which include only forecasts from the 21st of December 2020 on) are computed on different subsets. Numbers in brackets show the metrics relative to the Hub ensemble (i.e. the median ensemble of all other models submitted to the German and Polish Forecast Hub, excluding our contributions). WIS is the mean weighted interval score (lower values are better), WIS - sd is the standard deviation of all scores achieved by a model. Dispersion, over-prediction and under-prediction together sum up to the weighted interval score. Bias (between -1 and 1,0 is ideal) represents the general average tendency of a model to over- or underpredict. $50 \%$ and $90 \%$ coverage are the percentage of observed values that fell within the $50 \%$ and $90 \%$ prediction intervals of a model.

\begin{tabular}{|c|c|c|c|c|c|c|c|c|c|c|}
\hline & Model & WIS & WIS - sd & dispersion & Underpred. & Overpred. & Bias & Abs. error & $50 \%$-Cov. & $90 \%$-Cov. \\
\hline \multicolumn{11}{|l|}{ Cases } \\
\hline \multirow{4}{*}{3 wk ahead } & Hub-ensemble & $33400(1)$ & $40700(1)$ & $9130(1)$ & $7690(1)$ & $16600(1)$ & -0.01 & $46900(1)$ & 0.29 & 0.62 \\
\hline & Hub-ensemble-realised & $30800(0.92)$ & $38600(0.95)$ & $7910(0.87)$ & $6890(0.9)$ & $16000(0.96)$ & 0.03 & $44200(0.94)$ & 0.29 & 0.62 \\
\hline & Hub-ensemble-with-crowd & $30800(0.92)$ & $34100(0.84)$ & $7500(0.82)$ & $8960(1.17)$ & $14300(0.86)$ & 0.02 & $44100(0.94)$ & 0.24 & 0.55 \\
\hline & Hub-ensemble-with-renewal & $34000(1.02)$ & $43100(1.06)$ & $8860(0.97)$ & $6300(0.82)$ & $18900(1.14)$ & 0.02 & $48100(1.03)$ & 0.29 & 0.60 \\
\hline \multirow{4}{*}{4 wk ahead } & Hub-ensemble & $55900(1)$ & $73700(1)$ & $12200(1)$ & $12400(1)$ & $31300(1)$ & 0.01 & $74400(1)$ & 0.24 & 0.52 \\
\hline & Hub-ensemble-realised & $51200(0.92)$ & $69900(0.95)$ & $10900(0.89)$ & $11100(0.9)$ & $29300(0.94)$ & 0.04 & $69600(0.94)$ & 0.19 & 0.57 \\
\hline & Hub-ensemble-with-crowd & $48800(0.87)$ & $58600(0.8)$ & $9700(0.8)$ & $13700(1.1)$ & $25400(0.81)$ & 0.00 & $65800(0.88)$ & 0.19 & 0.48 \\
\hline & Hub-ensemble-with-renewal & $59100(1.06)$ & $84100(1.14)$ & $12600(1.03)$ & $10100(0.81)$ & $36400(1.16)$ & 0.01 & $78900(1.06)$ & 0.29 & 0.55 \\
\hline \multicolumn{11}{|l|}{ Deaths } \\
\hline \multirow{6}{*}{3 wk ahead } & Hub-ensemble & $319(1)$ & $328(1)$ & $172(1)$ & $92.7(1)$ & $55.1(1)$ & -0.03 & $488(1)$ & 0.54 & 0.96 \\
\hline & Hub-ensemble-realised & $332(1.04)$ & $388(1.18)$ & $158(0.92)$ & $78.7(0.85)$ & $95(1.72)$ & -0.02 & $547(1.12)$ & 0.46 & 1.00 \\
\hline & Hub-ensemble-with-all & $321(1.01)$ & $385(1.17)$ & $153(0.89)$ & $100(1.08)$ & $68.1(1.24)$ & -0.01 & $535(1.1)$ & 0.54 & 1.00 \\
\hline & Hub-ensemble-with-convolution & $298(0.93)$ & $337(1.03)$ & $155(0.9)$ & $106(1.14)$ & $37.5(0.68)$ & -0.04 & $441(0.9)$ & 0.67 & 0.92 \\
\hline & Hub-ensemble-with-crowd & $319(1)$ & $342(1.04)$ & $160(0.93)$ & $85.1(0.92)$ & $73.6(1.34)$ & -0.02 & $547(1.12)$ & 0.54 & 0.96 \\
\hline & Hub-ensemble-with-renewal & $332(1.04)$ & $363(1.11)$ & $168(0.98)$ & $86.1(0.93)$ & $78.2(1.42)$ & -0.02 & $528(1.08)$ & 0.58 & 0.96 \\
\hline \multirow{6}{*}{4 wk ahead } & Hub-ensemble & $424(1)$ & $443(1)$ & $212(1)$ & $126(1)$ & $85.7(1)$ & -0.06 & $675(1)$ & 0.58 & 0.92 \\
\hline & Hub-ensemble-realised & $445(1.05)$ & $532(1.2)$ & $193(0.91)$ & $107(0.85)$ & $144(1.68)$ & -0.03 & $700(1.04)$ & 0.54 & 0.92 \\
\hline & Hub-ensemble-with-all & $399(0.94)$ & $438(0.99)$ & $195(0.92)$ & $105(0.83)$ & $97.9(1.14)$ & -0.05 & $692(1.03)$ & 0.46 & 1.00 \\
\hline & Hub-ensemble-with-convolution & $384(0.91)$ & $387(0.87)$ & $196(0.92)$ & $122(0.97)$ & $65.9(0.77)$ & -0.06 & $602(0.89)$ & 0.54 & 0.96 \\
\hline & Hub-ensemble-with-crowd & $407(0.96)$ & $456(1.03)$ & $202(0.95)$ & $105(0.83)$ & $101(1.18)$ & -0.03 & $669(0.99)$ & 0.67 & 0.96 \\
\hline & Hub-ensemble-with-renewal & $457(1.08)$ & $527(1.19)$ & $208(0.98)$ & $129(1.02)$ & $121(1.41)$ & -0.06 & $744(1.1)$ & 0.50 & 0.96 \\
\hline
\end{tabular}


Table 6: Scores for one and two week ahead forecasts (cut to three significant digits and rounded) for the different versions of the mean ensemble. Note that scores for cases (which include the whole period from October 12th 2020 until March 1st 2021) and deaths (which include only forecasts from the 21st of December 2020 on) are computed on different subsets. Numbers in brackets show the metrics relative to the Hub mean ensemble (i.e. the mean ensemble of all other models submitted to the German and Polish Forecast Hub, excluding our contributions). WIS is the mean weighted interval score (lower values are better), WIS - sd is the standard deviation of all scores achieved by a model. Dispersion, over-prediction and under-prediction together sum up to the weighted interval score. Bias (between -1 and 1,0 is ideal) represents the general average tendency of a model to over- or underpredict. $50 \%$ and $90 \%$ coverage are the percentage of observed values that fell within the $50 \%$ and $90 \%$ prediction intervals of a model.

\begin{tabular}{|c|c|c|c|c|c|c|c|c|c|c|}
\hline & Model & WIS & WIS - sd & dispersion & Underpred. & Overpred. & Bias & Abs. error & $50 \%$-Cov. & $90 \%$-Cov. \\
\hline \multicolumn{11}{|l|}{ Cases } \\
\hline & Hub-ensemble-mean & $8680(1)$ & $10300(1)$ & $3700(1)$ & $1460(1)$ & $3520(1)$ & -0.02 & $13400(1)$ & 0.50 & 0.86 \\
\hline \multirow{3}{*}{1 wk ahead } & Hub-ensemble-realised-mean & $7600(0.88)$ & $8770(0.85)$ & $3360(0.91)$ & $1090(0.75)$ & $3140(0.89)$ & 0.01 & $11900(0.89)$ & 0.52 & 0.90 \\
\hline & Hub-ensemble-with-crowd-mean & $8050(0.93)$ & $9070(0.88)$ & $3520(0.95)$ & $1410(0.97)$ & $3120(0.89)$ & -0.02 & $12600(0.94)$ & 0.48 & 0.88 \\
\hline & Hub-ensemble-with-renewal-mean & $8090(0.93)$ & $9780(0.95)$ & $3490(0.94)$ & $1110(0.76)$ & $3490(0.99)$ & 0.02 & $12700(0.95)$ & 0.57 & 0.88 \\
\hline \multirow{4}{*}{2 wk ahead } & Hub-ensemble-mean & $19000(1)$ & $22100(1)$ & $5960(1)$ & $3690(1)$ & $9340(1)$ & 0.02 & $28800(1)$ & 0.33 & 0.79 \\
\hline & Hub-ensemble-realised-mean & $17100(0.9)$ & $20600(0.93)$ & $5550(0.93)$ & $2850(0.77)$ & $8660(0.93)$ & 0.05 & $26000(0.9)$ & 0.38 & 0.76 \\
\hline & Hub-ensemble-with-crowd-mean & $17600(0.93)$ & $20000(0.9)$ & $5540(0.93)$ & $3790(1.03)$ & $8230(0.88)$ & 0.01 & $26800(0.93)$ & 0.36 & 0.76 \\
\hline & Hub-ensemble-with-renewal-mean & $18300(0.96)$ & $22600(1.02)$ & $5910(0.99)$ & $2640(0.72)$ & $9720(1.04)$ & 0.06 & $27700(0.96)$ & 0.38 & 0.76 \\
\hline \multicolumn{11}{|l|}{ Deaths } \\
\hline \multirow{6}{*}{1 wk ahead } & Hub-ensemble-mean & $229(1)$ & $292(1)$ & $101(1)$ & $90.4(1)$ & $36.7(1)$ & -0.07 & $315(1)$ & 0.71 & 0.92 \\
\hline & Hub-ensemble-realised-mean & $219(0.96)$ & $289(0.99)$ & $96.8(0.96)$ & $79.8(0.88)$ & $42.6(1.16)$ & -0.04 & $297(0.94)$ & 0.71 & 0.88 \\
\hline & Hub-ensemble-with-all-mean & $217(0.95)$ & $287(0.98)$ & $95.3(0.94)$ & $83.1(0.92)$ & $38.7(1.05)$ & -0.07 & $300(0.95)$ & 0.67 & 0.88 \\
\hline & Hub-ensemble-with-convolution-mean & $225(0.98)$ & $292(1)$ & $98.7(0.98)$ & $94.2(1.04)$ & $32(0.87)$ & -0.09 & $314(1)$ & 0.71 & 0.92 \\
\hline & Hub-ensemble-with-crowd-mean & $222(0.97)$ & $289(0.99)$ & $98(0.97)$ & $84.1(0.93)$ & $39.6(1.08)$ & -0.04 & $295(0.94)$ & 0.71 & 0.88 \\
\hline & Hub-ensemble-with-renewal-mean & $225(0.98)$ & $290(0.99)$ & $99.7(0.99)$ & $84.7(0.94)$ & $40.5(1.1)$ & -0.05 & $314(1)$ & 0.71 & 0.88 \\
\hline \multirow{6}{*}{2 wk ahead } & Hub-ensemble-mean & $256(1)$ & $306(1)$ & $138(1)$ & $64.5(1)$ & $53.2(1)$ & 0.04 & $374(1)$ & 0.67 & 0.96 \\
\hline & Hub-ensemble-realised-mean & $270(1.05)$ & $338(1.1)$ & $136(0.99)$ & $65.2(1.01)$ & $68.1(1.28)$ & 0.06 & $413(1.1)$ & 0.75 & 0.92 \\
\hline & Hub-ensemble-with-all-mean & $268(1.05)$ & $346(1.13)$ & $133(0.96)$ & $78.7(1.22)$ & $57.1(1.07)$ & 0.05 & $408(1.09)$ & 0.75 & 0.96 \\
\hline & Hub-ensemble-with-convolution-mean & $259(1.01)$ & $322(1.05)$ & $133(0.96)$ & $81.7(1.27)$ & $44.4(0.83)$ & 0.03 & $380(1.02)$ & 0.67 & 0.96 \\
\hline & Hub-ensemble-with-crowd-mean & $264(1.03)$ & $315(1.03)$ & $133(0.96)$ & $70.1(1.09)$ & $60(1.13)$ & 0.06 & $404(1.08)$ & 0.71 & 0.96 \\
\hline & Hub-ensemble-with-renewal-mean & $264(1.03)$ & $332(1.08)$ & $141(1.02)$ & $60.1(0.93)$ & $63.1(1.19)$ & 0.06 & $390(1.04)$ & 0.79 & 0.92 \\
\hline
\end{tabular}


Table 7: Scores for three and four week ahead forecasts (cut to three significant digits and rounded) for the different versions of the mean ensemble. Note that scores for cases (which include the whole period from October 12th 2020 until March 1st 2021) and deaths (which include only forecasts from the 21st of December 2020 on) are computed on different subsets. Numbers in brackets show the metrics relative to the Hub mean ensemble (i.e. the mean ensemble of all other models submitted to the German and Polish Forecast Hub, excluding our contributions). WIS is the mean weighted interval score (lower values are better), WIS - sd is the standard deviation of all scores achieved by a model. Dispersion, over-prediction and under-prediction together sum up to the weighted interval score. Bias (between -1 and 1,0 is ideal) represents the general average tendency of a model to over- or underpredict. $50 \%$ and $90 \%$ coverage are the percentage of observed values that fell within the $50 \%$ and $90 \%$ prediction intervals of a model.

\begin{tabular}{|c|c|c|c|c|c|c|c|c|c|c|}
\hline & Model & WIS & WIS - sd & dispersion & Underpred. & Overpred. & Bias & Abs. error & $50 \%$-Cov. & $90 \%$-Cov. \\
\hline \multicolumn{11}{|l|}{ Cases } \\
\hline \multirow{4}{*}{3 wk ahead } & Hub-ensemble-mean & $35600(1)$ & $42100(1)$ & $9340(1)$ & $7050(1)$ & $19200(1)$ & 0.03 & $51200(1)$ & 0.26 & 0.62 \\
\hline & Hub-ensemble-realised-mean & $32100(0.9)$ & $40500(0.96)$ & $8830(0.95)$ & $4920(0.7)$ & $18300(0.95)$ & 0.07 & $47200(0.92)$ & 0.29 & 0.64 \\
\hline & Hub-ensemble-with-crowd-mean & $32200(0.9)$ & $36700(0.87)$ & $8430(0.9)$ & $7190(1.02)$ & $16500(0.86)$ & 0.04 & $46900(0.92)$ & 0.24 & 0.64 \\
\hline & Hub-ensemble-with-renewal-mean & $35200(0.99)$ & $46000(1.09)$ & $9630(1.03)$ & $4600(0.65)$ & $20900(1.09)$ & 0.08 & $51000(1)$ & 0.38 & 0.67 \\
\hline \multirow{4}{*}{4 wk ahead } & Hub-ensemble-mean & $60300(1)$ & $79300(1)$ & $15700(1)$ & $10400(1)$ & $34100(1)$ & 0.04 & $78600(1)$ & 0.29 & 0.57 \\
\hline & Hub-ensemble-realised-mean & $55000(0.91)$ & $77100(0.97)$ & $14600(0.93)$ & $6620(0.64)$ & $33800(0.99)$ & 0.11 & $75200(0.96)$ & 0.33 & 0.64 \\
\hline & Hub-ensemble-with-crowd-mean & $53400(0.89)$ & $66600(0.84)$ & $13700(0.87)$ & $10600(1.02)$ & $29200(0.86)$ & 0.06 & $70400(0.9)$ & 0.26 & 0.60 \\
\hline & Hub-ensemble-with-renewal-mean & $61700(1.02)$ & $89800(1.13)$ & $16400(1.04)$ & $6400(0.62)$ & $38900(1.14)$ & 0.12 & $82900(1.05)$ & 0.31 & 0.64 \\
\hline \multicolumn{11}{|l|}{ Deaths } \\
\hline & Hub-ensemble-mean & $289(1)$ & $293(1)$ & $178(1)$ & $45.9(1)$ & $65.7(1)$ & 0.01 & $443(1)$ & 0.58 & 1.00 \\
\hline \multirow{5}{*}{3 wk ahead } & Hub-ensemble-realised-mean & $310(1.07)$ & $348(1.19)$ & $182(1.02)$ & $42(0.92)$ & $86.5(1.32)$ & 0.08 & $502(1.13)$ & 0.58 & 1.00 \\
\hline & Hub-ensemble-with-all-mean & $315(1.09)$ & $339(1.16)$ & $178(1)$ & $62.2(1.36)$ & $74(1.13)$ & 0.07 & $507(1.14)$ & 0.62 & 1.00 \\
\hline & Hub-ensemble-with-convolution-mean & $297(1.03)$ & $292(1)$ & $174(0.98)$ & $67.7(1.47)$ & $55(0.84)$ & 0.01 & $452(1.02)$ & 0.67 & 1.00 \\
\hline & Hub-ensemble-with-crowd-mean & $294(1.02)$ & $299(1.02)$ & $172(0.97)$ & $48(1.05)$ & $74.2(1.13)$ & 0.03 & $476(1.07)$ & 0.58 & 1.00 \\
\hline & Hub-ensemble-with-renewal-mean & $310(1.07)$ & $349(1.19)$ & $189(1.06)$ & $39.4(0.86)$ & $81.9(1.25)$ & 0.05 & $482(1.09)$ & 0.62 & 1.00 \\
\hline \multirow{6}{*}{4 wk ahead } & Hub-ensemble-mean & $437(1)$ & $568(1)$ & $232(1)$ & $72(1)$ & $134(1)$ & 0.00 & $702(1)$ & 0.62 & 1.00 \\
\hline & Hub-ensemble-realised-mean & $445(1.02)$ & $598(1.05)$ & $237(1.02)$ & $56.4(0.78)$ & $152(1.13)$ & 0.06 & $707(1.01)$ & 0.58 & 1.00 \\
\hline & Hub-ensemble-with-all-mean & $421(0.96)$ & $520(0.92)$ & $239(1.03)$ & $49.9(0.69)$ & $132(0.99)$ & 0.05 & $678(0.97)$ & 0.58 & 1.00 \\
\hline & Hub-ensemble-with-convolution-mean & $398(0.91)$ & $465(0.82)$ & $235(1.01)$ & $55.6(0.77)$ & $107(0.8)$ & 0.00 & $628(0.89)$ & 0.67 & 1.00 \\
\hline & Hub-ensemble-with-crowd-mean & $418(0.96)$ & $533(0.94)$ & $222(0.96)$ & $66.8(0.93)$ & $129(0.96)$ & 0.03 & $662(0.94)$ & 0.58 & 1.00 \\
\hline & Hub-ensemble-with-renewal-mean & $467(1.07)$ & $636(1.12)$ & $248(1.07)$ & $61(0.85)$ & $158(1.18)$ & 0.05 & $755(1.08)$ & 0.67 & 1.00 \\
\hline
\end{tabular}




\section{A.11 Sensitivity analysis}

In the original analysis, cases and deaths were scored on different periods, as the convolution model was only added later. This sensitivity shows performance of all models restricted to the period from October 142020 until March 1st 2021 where all models were available.

A

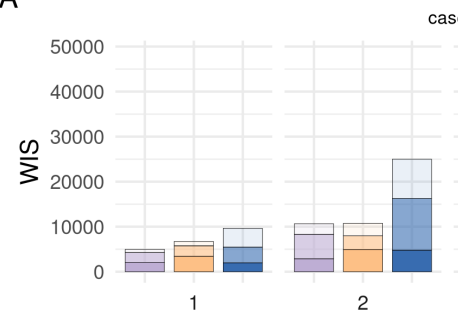

C

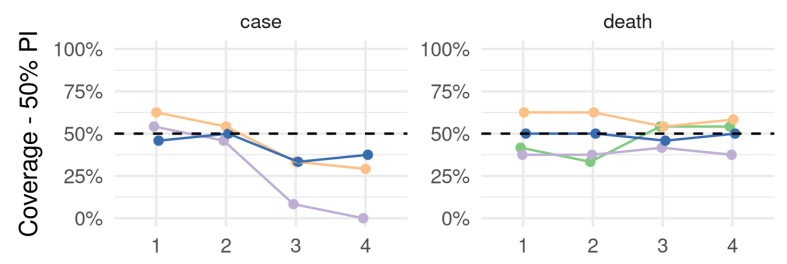

$\mathrm{E}$

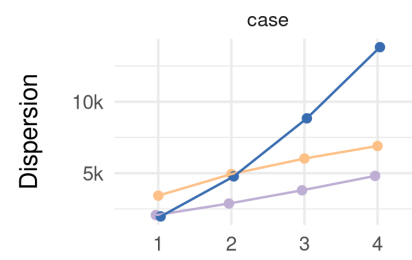

$\mathrm{G}$

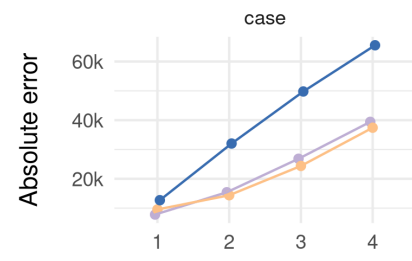

case

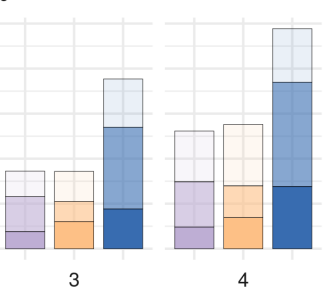

B

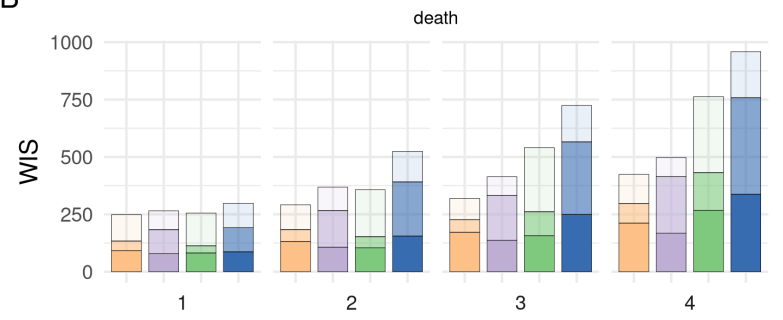

$\mathrm{D}$

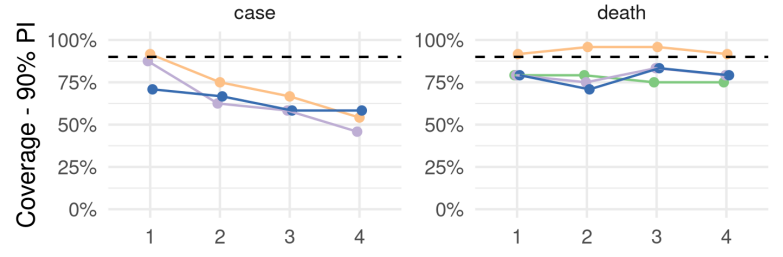

$\mathrm{F}$
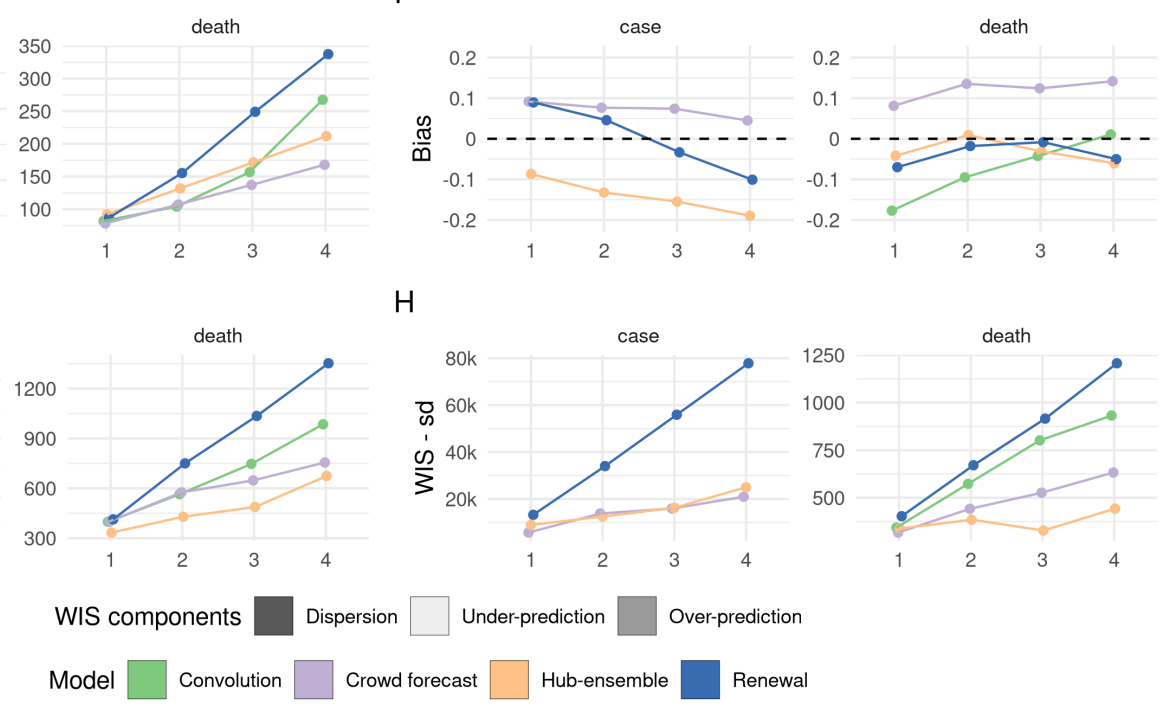

$\mathrm{H}$
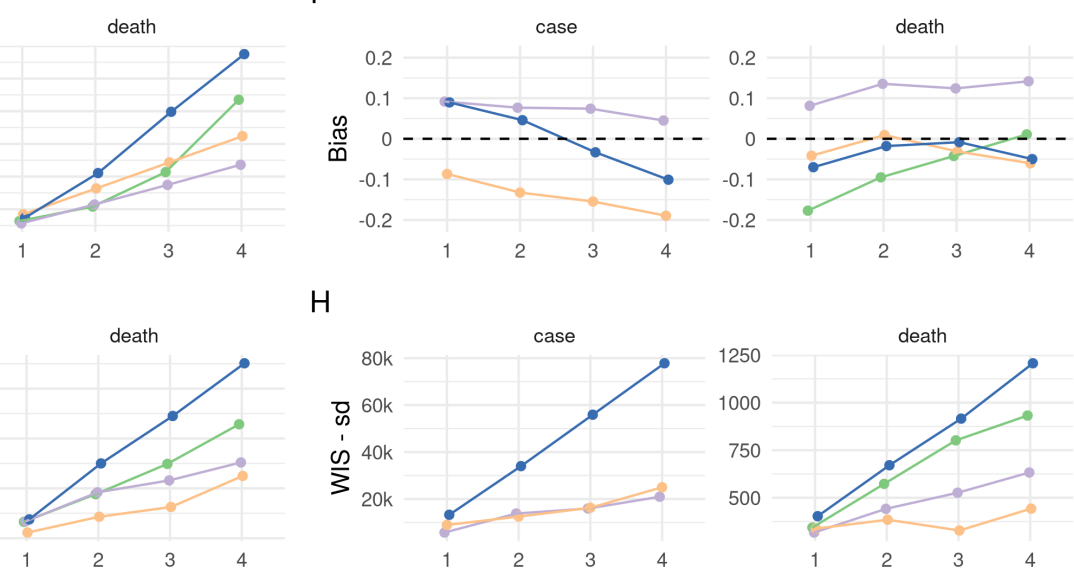

Figure 20: Visualisation of aggregate performance metrics across forecast horizons only for the period from October 14th 2020 on where all models were available. A, B: mean weighted interval score (WIS, lower indicates better performance) across horizons. WIS is decomposed into its components dispersion, over-prediction and under-prediction. C: Empirical coverage of the $50 \%$ prediction intervals (50\% coverage is perfect). D: Empirical coverage of the 90\% prediction intervals. E: Dispersion (same as in panel A, B). Higher values mean greater dispersion of the forecast and imply ceteris paribus a worse score. F: Bias, i.e. general (relative) tendency to over- or underpredict. Values are between -1 (complete under-prediction) and 1 (complete over-prediction) and 0 ideally. G: Absolute error of the median forecast (lower is better). H. Standard deviation of all WIS values for different horizons 
Table 8: Scores for one and two week ahead forecasts (cut to three significant digits and rounded) calculated on forecasts made between December 14th 2020 and March 1st 2021. Numbers in brackets show the metrics relative to the Hub ensemble (i.e. the median ensemble of all other models submitted to the German and Polish Forecast Hub, excluding our contributions). WIS is the mean weighted interval score (lower values are better), WIS - sd is the standard deviation of all scores achieved by a model. Dispersion, over-prediction and under-prediction together sum up to the weighted interval score. Bias (between -1 and 1,0 is ideal) represents the general average tendency of a model to over- or underpredict. $50 \%$ and $90 \%$ coverage are the percentage of observed values that fell within the $50 \%$ and $90 \%$ prediction intervals of a model.

\begin{tabular}{|c|c|c|c|c|c|c|c|c|c|c|}
\hline & Model & WIS & WIS - sd & dispersion & Underpred. & Overpred. & Bias & Abs. error & $50 \%$-Cov. & $90 \%$-Cov \\
\hline \multicolumn{11}{|l|}{ Cases } \\
\hline & Crowd forecast & $4980(0.74)$ & $5730(0.64)$ & $2070(0.6)$ & $728(0.74)$ & $2190(0.94)$ & 0.09 & $7810(0.82)$ & 0.54 & 0.88 \\
\hline \multirow{2}{*}{1 wk ahead } & Hub-ensemble & $6730(1)$ & $8960(1)$ & $3430(1)$ & $978(1)$ & $2330(1)$ & -0.09 & $9550(1)$ & 0.62 & 0.92 \\
\hline & Renewal & $9640(1.43)$ & $13300(1.48)$ & $1970(0.57)$ & $4170(4.26)$ & $3500(1.5)$ & 0.09 & $12700(1.33)$ & 0.46 & 0.71 \\
\hline \multirow{3}{*}{2 wk ahead } & Crowd forecast & $10700(0.99)$ & $13800(1.1)$ & $2880(0.58)$ & $2350(0.85)$ & $5430(1.79)$ & 0.08 & $15400(1.07)$ & 0.46 & 0.62 \\
\hline & Hub-ensemble & $10800(1)$ & $12500(1)$ & $4940(1)$ & $2780(1)$ & $3030(1)$ & -0.13 & $14400(1)$ & 0.54 & 0.75 \\
\hline & Renewal & $25000(2.31)$ & $34000(2.72)$ & $4780(0.97)$ & $8710(3.13)$ & $11500(3.8)$ & 0.05 & $32000(2.22)$ & 0.50 & 0.67 \\
\hline \multicolumn{11}{|l|}{ Deaths } \\
\hline \multirow{4}{*}{1 wk ahead } & Convolution & $255(1.03)$ & $343(1.01)$ & $82(0.89)$ & $142(1.23)$ & $31.1(0.75)$ & -0.18 & $399(1.19)$ & 0.42 & 0.79 \\
\hline & Crowd forecast & $265(1.07)$ & $317(0.94)$ & $78.2(0.85)$ & $82(0.71)$ & $105(2.52)$ & 0.08 & $402(1.2)$ & 0.38 & 0.79 \\
\hline & Hub-ensemble & $248(1)$ & $338(1)$ & $92.2(1)$ & $115(1)$ & $41.6(1)$ & -0.04 & $334(1)$ & 0.62 & 0.92 \\
\hline & Renewal & $298(1.2)$ & $403(1.19)$ & $87(0.94)$ & $107(0.93)$ & $105(2.52)$ & -0.07 & $413(1.24)$ & 0.50 & 0.79 \\
\hline \multirow{4}{*}{2 wk ahead } & Convolution & $357(1.22)$ & $573(1.49)$ & $104(0.79)$ & $204(1.89)$ & $48.8(0.94)$ & -0.10 & $565(1.32)$ & 0.33 & 0.79 \\
\hline & Crowd forecast & $368(1.26)$ & $442(1.15)$ & $107(0.81)$ & $102(0.94)$ & $160(3.08)$ & 0.14 & $576(1.34)$ & 0.38 & 0.75 \\
\hline & Hub-ensemble & $292(1)$ & $385(1)$ & $132(1)$ & $108(1)$ & $51.9(1)$ & 0.01 & $429(1)$ & 0.62 & 0.96 \\
\hline & Renewal & $524(1.79)$ & $671(1.74)$ & $155(1.17)$ & $133(1.23)$ & $236(4.55)$ & -0.02 & $750(1.75)$ & 0.50 & 0.71 \\
\hline
\end{tabular}

Table 9: Scores for three and four week ahead forecasts (cut to three significant digits and rounded) calculated on forecasts made between December 14th 2020 and March 1st 2021. Numbers in brackets show the metrics relative to the Hub ensemble (i.e. the median ensemble of all other models submitted to the German and Polish Forecast Hub, excluding our contributions). WIS is the mean weighted interval score (lower values are better), WIS - sd is the standard deviation of all scores achieved by a model. Dispersion, over-prediction and under-prediction together sum up to the weighted interval score. Bias (between -1 and 1,0 is ideal) represents the general average tendency of a model to over- or underpredict. $50 \%$ and $90 \%$ coverage are the percentage of observed values that fell within the $50 \%$ and $90 \%$ prediction intervals of a model.

\begin{tabular}{|c|c|c|c|c|c|c|c|c|c|c|}
\hline & Model & WIS & WIS - sd & dispersion & Underpred. & Overpred. & Bias & Abs. error & $50 \%$-Cov. & $90 \%$-Cov. \\
\hline \multicolumn{11}{|l|}{ Cases } \\
\hline \multirow{3}{*}{3 wk ahead } & Crowd forecast & $17200(1)$ & $16000(0.98)$ & $3800(0.63)$ & $5660(0.85)$ & $7770(1.74)$ & 0.07 & $26800(1.1)$ & 0.08 & 0.58 \\
\hline & Hub-ensemble & $17200(1)$ & $16300(1)$ & $6030(1)$ & $6670(1)$ & $4470(1)$ & -0.16 & $24400(1)$ & 0.33 & 0.67 \\
\hline & Renewal & $37700(2.19)$ & $55900(3.43)$ & $8840(1.47)$ & $10700(1.6)$ & $18100(4.05)$ & -0.03 & $49800(2.04)$ & 0.33 & 0.58 \\
\hline \multirow{3}{*}{4 wk ahead } & Crowd forecast & $26100(0.95)$ & $21000(0.84)$ & $4810(0.7)$ & $11300(0.83)$ & $10100(1.43)$ & 0.04 & $39400(1.05)$ & 0.00 & 0.46 \\
\hline & Hub-ensemble & $27600(1)$ & $25000(1)$ & $6900(1)$ & $13600(1)$ & $7060(1)$ & -0.19 & $37400(1)$ & 0.29 & 0.54 \\
\hline & Renewal & $48900(1.77)$ & $77800(3.11)$ & $13800(2)$ & $11900(0.88)$ & $23200(3.29)$ & -0.10 & $65500(1.75)$ & 0.38 & 0.58 \\
\hline \multicolumn{11}{|l|}{ Deaths } \\
\hline \multirow{4}{*}{3 wk ahead } & Convolution & $541(1.7)$ & $802(2.45)$ & $157(0.91)$ & $279(3.01)$ & $105(1.91)$ & -0.04 & $747(1.53)$ & 0.54 & 0.75 \\
\hline & Crowd forecast & $414(1.3)$ & $526(1.6)$ & $137(0.8)$ & $82(0.88)$ & $194(3.52)$ & 0.12 & $648(1.33)$ & 0.42 & 0.83 \\
\hline & Hub-ensemble & $319(1)$ & $328(1)$ & $172(1)$ & $92.7(1)$ & $55.1(1)$ & -0.03 & $488(1)$ & 0.54 & 0.96 \\
\hline & Renewal & $724(2.27)$ & $916(2.79)$ & $249(1.45)$ & $158(1.7)$ & $317(5.75)$ & -0.01 & $1040(2.13)$ & 0.46 & 0.83 \\
\hline \multirow{4}{*}{4 wk ahead } & Convolution & $763(1.8)$ & $932(2.1)$ & $268(1.26)$ & $331(2.63)$ & $164(1.91)$ & 0.01 & $985(1.46)$ & 0.54 & 0.75 \\
\hline & Crowd forecast & $498(1.17)$ & $633(1.43)$ & $168(0.79)$ & $83.6(0.66)$ & $246(2.87)$ & 0.14 & $756(1.12)$ & 0.38 & 0.79 \\
\hline & Hub-ensemble & $424(1)$ & $443(1)$ & $212(1)$ & $126(1)$ & $85.7(1)$ & -0.06 & $675(1)$ & 0.58 & 0.92 \\
\hline & Renewal & $959(2.26)$ & $1210(2.73)$ & $337(1.59)$ & $200(1.59)$ & $421(4.91)$ & -0.05 & $1350(2)$ & 0.50 & 0.79 \\
\hline
\end{tabular}




\section{A.12 Overview of models and forecasters}

Table 10: Overview of the models and ensembles used.

\begin{tabular}{ll}
\hline Name & Explanation \\
\hline Hub-ensemble-realised & Official Forecast Hub median ensemble. Created by the Forecast Hub \\
& officially under the name 'KITCOVIDhub-median_ensemble' and used \\
& as the default ensemble. Included are our crowd forecasts as well as the \\
& renewal model (with one missed submission on December 28 2020, but \\
& not the convolution model which was deemed to similar to the renewal \\
& model.
\end{tabular}

Hub-ensemble-realised-mean

Official Forecast Hub mean ensemble. Created by the Forecast Hub officially under the name 'KITCOVIDhub-mean_ensemble'.

Hub-ensemble

Hub-ensemble-mean

Hub-ensemble-with-renewal, Hub-ensemble-with-renewalmean

Hub-ensemble-with-crowd, Hub-ensemble-with-crowdmean

Hub-ensemble-withconvolution, Hub-ensemble-withconvolution-mean

Hub-ensemble-with-all, Hub-ensemble-with-all-mean

Crowd forecast

Renewal model

Convolution model
Version of the official Hub median ensemble which excludes all our contributions.

Version of the official Hub mean ensemble which excludes all our contributions.

Versions of the official Hub ensembles which of our contributions includes only the Renewal model.

Versions of the official Hub ensembles which of our contributions includes only the Crowd forecast.

Versions of the official Hub ensembles which of our contributions includes only the Convolution model (which originally was never included in any official Hub ensemble).

Versions of the official Hub ensembles which includes all our contributions. For cases, this is identical to the official Hub ensembles, but for deaths the convolution model was added.

Submitted to the Forecast Hub as 'epiforecasts-EpiExpert'

Submitted to the Forecast Hub as 'epiforecasts-EpiNow2'

Submitted to the Forecast Hub as 'epiforecasts-EpiNow2_secondary' 
medRxiv preprint doi: https://doi.org/10.1101/2021.12.01.21266598; this version posted December 5, 2021. The copyright holder for this preprint (which was not certified by peer review) is the author/funder, who has granted medRxiv a license to display the preprint in perpetuity.

It is made available under a CC-BY 4.0 International license.

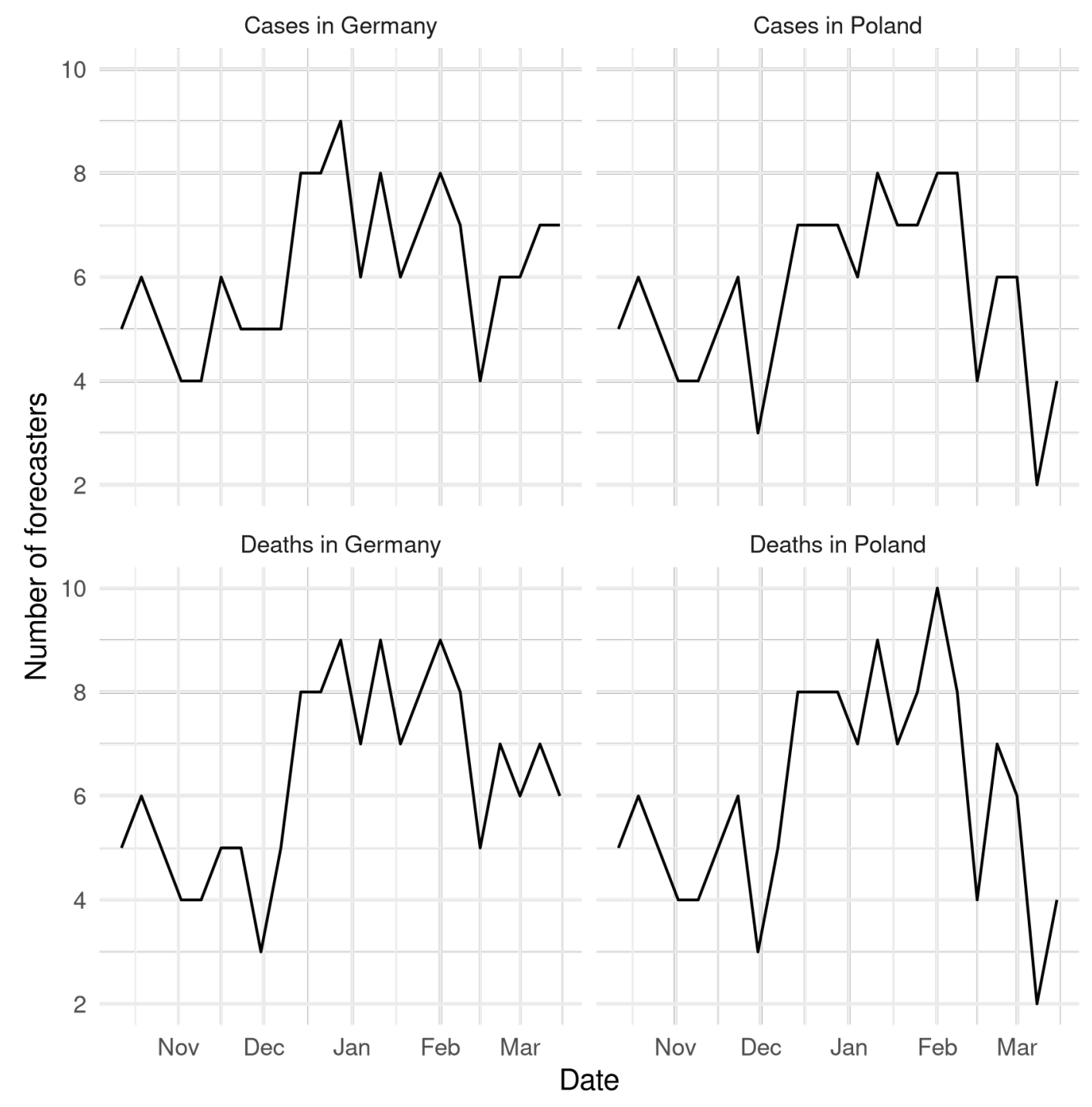

Figure 21: Number of participants who submitted a forecast over time. 


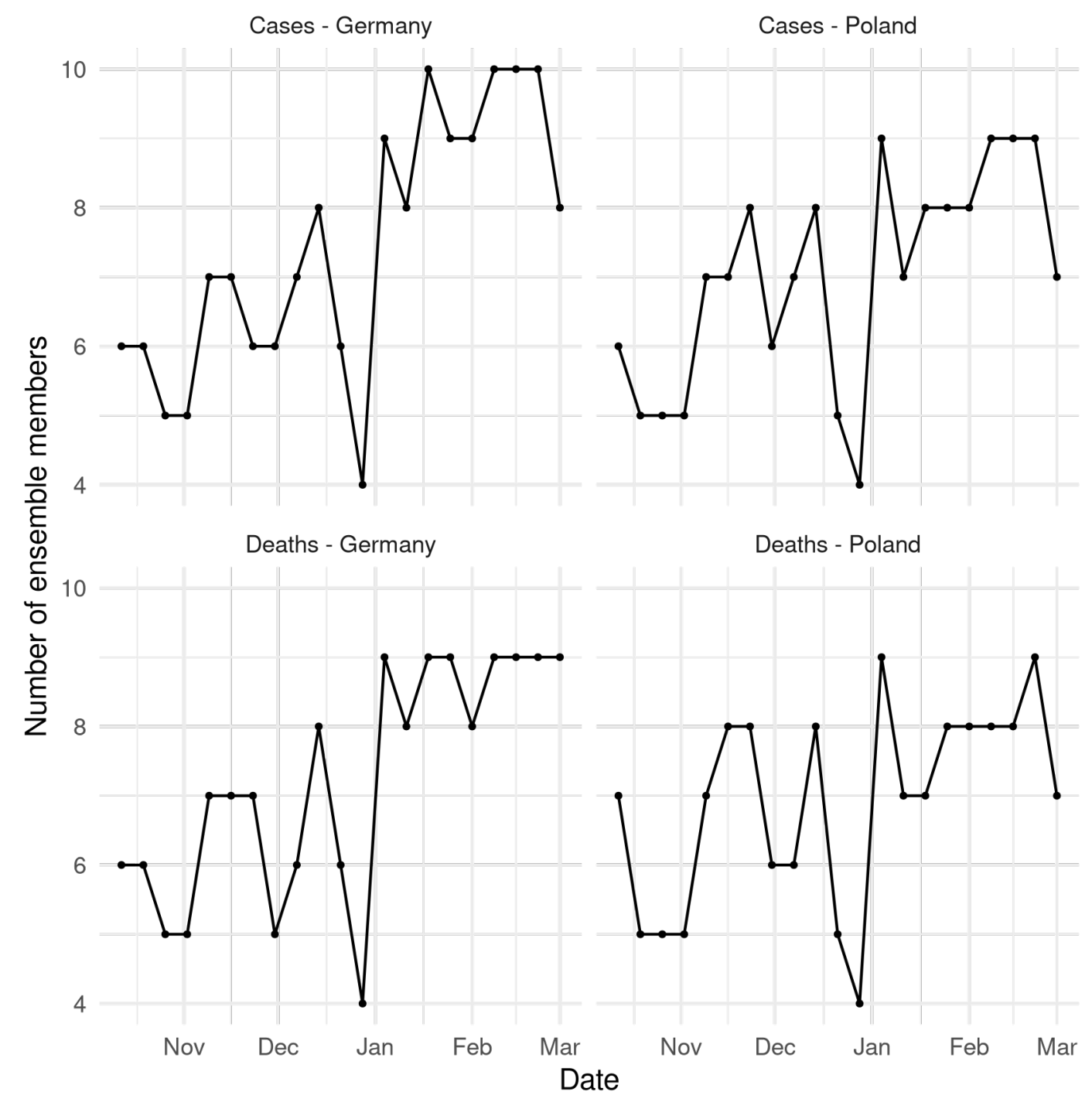

Figure 22: Number of member models (including our crowd forecasts and the renewal model) in the official Hub ensemble. Note that the renewal model was not included in the ensemble on December 28th 2020. 
Abbott, S., Hellewell, J., Hickson, J., Munday, J., Gostic, K., Ellis, P., Sherratt, K., Gibbs, H., Thompson, R., Meakin, S., Bosse, N. I., Mee, P., \& Funk, S. (2020). EpiNow2: Estimate real-time case counts and time-varying epidemiological parameters. -, -(-), -. https://doi.org/10.5281/zenodo.3957489

Abbott, S., Hellewell, J., Thompson, R. N., Sherratt, K., Gibbs, H. P., Bosse, N. I., Munday, J. D., Meakin, S., Doughty, E. L., Chun, J. Y., Chan, Y.-W. D., Finger, F., Campbell, P., Endo, A., Pearson, C. A. B., Gimma, A., Russell, T., Group, C. C. modelling, Flasche, S., .. F Funk, S. (2020). Estimating the time-varying reproduction number of SARS-CoV-2 using national and subnational case counts. 5:112. https://doi.org/10.12688/wellcomeopenres.16006.1

Abbott, S., Sherratt, K., Bevan, J., Gibbs, H., Hellewell, J., Munday, J., Barks, P., Campbell, P., Finger, F., \& Funk, S. (2020). Covidregionaldata: Subnational data for the covid-19 outbreak. -, -(-), -. https://doi.org/10.5281/zenodo.3957539

Ärzteblatt, D. Ä. G., Redaktion Deutsches. (2020, November 3). SARS-CoV-2-Diagnostik: RKI passt Testempfehlungen an. Deutsches Ärzteblatt. https://www.aerzteblatt.de/nachrichten/118001/SARSCoV-2-Diagnostik-RKI-passt-Testempfehlungen-an

Atanasov, P., Rescober, P., Stone, E., Swift, S. A., Servan-Schreiber, E., Tetlock, P., Ungar, L., \& Mellers, B. (2016). Distilling the Wisdom of Crowds: Prediction Markets vs. Prediction Polls. Management Science, 63(3), 691-706. https://doi.org/10.1287/mnsc.2015.2374

Bhatt, S., Ferguson, N., Flaxman, S., Gandy, A., Mishra, S., \& Scott, J. A. (n.d.). Semi-Mechanistic Bayesian modeling of COVID-19 with Renewal Processes. 14.

Biggerstaff, M., Alper, D., Dredze, M., Fox, S., Fung, I. C.-H., Hickmann, K. S., Lewis, B., Rosenfeld, R., Shaman, J., Tsou, M.-H., Velardi, P., Vespignani, A., Finelli, L., \& for the Influenza Forecasting Contest Working Group. (2016). Results from the centers for disease control and prevention's predict the 2013-2014 Influenza Season Challenge. BMC Infectious Diseases, 16(1), 357. https://doi.org/10.1186/s12879-016$1669-\mathrm{x}$

Bosse, N. I., Abbott, S., EpiForecasts, \& Funk, S. (2020a). Covid.german.forecasts: Forecasting covid-19 related metrics for the german/poland forecast hub.

Bosse, N. I., Abbott, S., EpiForecasts, \& Funk, S. (2020b). Crowdforecastr: Eliciting crowd forecasts in $r$ shiny. https://doi.org/10.5281/zenodo.4618519

Bosse, N. I., Abbott, S., EpiForecasts, \& Funk, S. (2020c). Scoringutils: Utilities for scoring and assessing predictions. https://doi.org/10.5281/zenodo.4618017

Bracher, J., Ray, E. L., Gneiting, T., \& Reich, N. G. (2021). Evaluating epidemic forecasts in an interval format. PLoS Computational Biology, 17(2), e1008618. https://doi.org/10.1371/journal.pcbi.1008618

Bracher, J., Wolffram, D., Deuschel, J., Görgen, K., Ketterer, J. L., Ullrich, A., Abbott, S., Barbarossa, M. V., Bertsimas, D., Bhatia, S., Bodych, M., Bosse, N. I., Burgard, J. P., Castro, L., Fairchild, G., Fuhrmann, J., Funk, S., Gogolewski, K., Gu, Q., ... Schienle, M. (2021). Short-term forecasting of COVID-19 in Germany and Poland during the second wave - a preregistered study. medRxiv, 2020.12.24.20248826. https://doi.org/10.1101/2020.12.24.20248826

Bracher, J., Wolffram, D., Deuschel, J., Görgen, K., Ketterer, J. L., Ullrich, A., Abbott, S., Barbarossa, M. V., Bertsimas, D., Bhatia, S., Bodych, M., Bosse, N. I., Burgard, J. P., Fiedler, J., Fuhrmann, J., Funk, S., Gambin, A., Gogolewski, K., Heyder, S., ... Schienle, M. (2021). National and subnational short-term forecasting of COVID-19 in Germany and Poland, early 2021. 2021.11.05.21265810. https: //doi.org/10.1101/2021.11.05.21265810

Chang, W., Cheng, J., Allaire, J., Sievert, C., Schloerke, B., Xie, Y., Allen, J., McPherson, J., Dipert, A., \& Borges, B. (2021). Shiny: Web application framework for $r$. https://CRAN.R-project.org/package=shiny

Colón-González, F. J., Bastos, L. S., Hofmann, B., Hopkin, A., Harpham, Q., Crocker, T., Amato, R., Ferrario, I., Moschini, F., James, S., Malde, S., Ainscoe, E., Nam, V. S., Tan, D. Q., Khoa, N. D., Harrison, M., Tsarouchi, G., Lumbroso, D., Brady, O. J., \& Lowe, R. (2021). Probabilistic seasonal dengue forecasting in Vietnam: A modelling study using superensembles. PLOS Medicine, 18(3), e1003542. https://doi.org/10.1371/journal.pmed.1003542

Cramer, E., Ray, E. L., Lopez, V. K., Bracher, J., Brennen, A., Rivadeneira, A. J. C., Gerding, A., Gneiting, T., House, K. H., Huang, Y., Jayawardena, D., Kanji, A. H., Khandelwal, A., Le, K., Mühlemann, A., Niemi, J., Shah, A., Stark, A., Wang, Y., .. Reich, N. G. (2021). Evaluation of individual and ensemble probabilistic forecasts of COVID-19 mortality in the US. medRxiv, 2021.02.03.21250974. https://doi.org/10.1101/2021.02.03.21250974 
Cramer, E., Reich, N. G., Wang, S. Y., Niemi, J., Hannan, A., House, K., Gu, Y., Xie, S., Horstman, S., aniruddhadiga, Walraven, R., starkari, Li, M. L., Gibson, G., Castro, L., Karlen, D., Wattanachit, N., jinghuichen, zyt9lsb, ... leyouz. (2020). COVID-19 Forecast Hub: 4 December 2020 snapshot [Data set]. Zenodo. https://doi.org/10.5281/zenodo.3963371

CSET Foretell. (2021). CSET Foretell. https://www.cset-foretell.com/

Del Valle, S. Y., McMahon, B. H., Asher, J., Hatchett, R., Lega, J. C., Brown, H. E., Leany, M. E., Pantazis, Y., Roberts, D. J., Moore, S., Peterson, A. T., Escobar, L. E., Qiao, H., Hengartner, N. W., \& Mukundan, H. (2018). Summary results of the 2014-2015 DARPA Chikungunya challenge. BMC Infectious Diseases, 18(1), 245. https://doi.org/10.1186/s12879-018-3124-7

Deutsche Welle. (2020). Coronavirus: Germany to impose one-month partial lockdown / DW / 28.10.2020. https://www.dw.com/en/coronavirus-germany-to-impose-one-month-partial-lockdown/a-55421241

ECDC. (2020, December 14). Download historical data (to 14 December 2020) on the daily number of new reported COVID-19 cases and deaths worldwide. European Centre for Disease Prevention and Control. https://www.ecdc.europa.eu/en/publications-data/download-todays-data-geographic-distributioncovid-19-cases-worldwide

epiforecasts.io/covid. (2020). Covid-19: Temporal variation in transmission during the COVID-19 outbreak. Covid-19. https://epiforecasts.io/covid/

European Covid-19 Forecast Hub. (2021). European Covid-19 Forecast Hub. https://covid19forecasthub.eu/

Evaluating the use of the reproduction number as an epidemiological tool, using spatio-temporal trends of the Covid-19 outbreak in England / medRxiv. (n.d.). Retrieved May 30, 2021, from https://www.medrxiv.org/ content/10.1101/2020.10.18.20214585v1

Farrow, D. C., Brooks, L. C., Hyun, S., Tibshirani, R. J., Burke, D. S., \& Rosenfeld, R. (2017). A human judgment approach to epidemiological forecasting. PLOS Computational Biology, $13(3)$, e1005248. https://doi.org/10.1371/journal.pcbi.1005248

Fay, C., Guyader, V., Rochette, S., \& Girard, C. (2021). Golem: A framework for robust shiny applications. https://github.com/ThinkR-open/golem

Forsal.pl. (2020). Rozbieżności w statystykach koronawirusa. 22 tys. przypadków będa doliczone do ogólnej liczby wyników. https://forsal.pl/lifestyle/zdrowie/artykuly/8017628,rozbieznosci-w-statystykachkoronawirusa-22-tys-przypadkow-beda-doliczone-do-ogolnej-liczby-wynikow.html

Fraser, C. (2007). Estimating Individual and Household Reproduction Numbers in an Emerging Epidemic. PLOS ONE, 2(8), e758. https://doi.org/10.1371/journal.pone.0000758

Funk, S., Abbott, S., Atkins, B. D., Baguelin, M., Baillie, J. K., Birrell, P., Blake, J., Bosse, N. I., Burton, J., Carruthers, J., Davies, N. G., Angelis, D. D., Dyson, L., Edmunds, W. J., Eggo, R. M., Ferguson, N. M., Gaythorpe, K., Gorsich, E., Guyver-Fletcher, G., . . Investigators, I. (2020). Short-term forecasts to inform the response to the Covid-19 epidemic in the UK. medRxiv, 2020.11.11.20220962. https://doi.org/10.1101/2020.11.11.20220962

Funk, S., Camacho, A., Kucharski, A. J., Lowe, R., Eggo, R. M., \& Edmunds, W. J. (2019). Assessing the performance of real-time epidemic forecasts: A case study of Ebola in the Western Area region of Sierra Leone, 2014-15. PLOS Computational Biology, 15(2), e1006785. https://doi.org/10.1371/journal.pcbi.100 6785

Ganyani, T., Kremer, C., Chen, D., Torneri, A., Faes, C., Wallinga, J., \& Hens, N. (2020). Estimating the generation interval for coronavirus disease (COVID-19) based on symptom onset data, march 2020. Eurosurveillance, 25(17).

Gneiting, T., \& Raftery, A. E. (2007). Strictly proper scoring rules, prediction, and estimation. Journal of the American Statistical Association, 102(477), 359-378. https://doi.org/10.1198/016214506000001437

Held, L., Meyer, S., \& Bracher, J. (2017). Probabilistic forecasting in infectious disease epidemiology: The 13th Armitage lecture. Statistics in Medicine, 36(22), 3443-3460. https://doi.org/10.1002/sim.7363

Hoogeveen, S., Sarafoglou, A., \& Wagenmakers, E.-J. (2020). Laypeople Can Predict Which Social-Science Studies Will Be Replicated Successfully. Advances in Methods and Practices in Psychological Science, 3(3), 267-285. https://doi.org/10.1177/2515245920919667

Hypermind. (2021). Hypermind / Supercollective intelligence for decision makers. Hypermind. https: //www.hypermind.com/en/

Johansson, M. A., Apfeldorf, K. M., Dobson, S., Devita, J., Buczak, A. L., Baugher, B., Moniz, L. J., Bagley, T., Babin, S. M., Guven, E., Yamana, T. K., Shaman, J., Moschou, T., Lothian, N., Lane, A., Osborne, 
G., Jiang, G., Brooks, L. C., Farrow, D. C., .. Chretien, J.-P. (2019). An open challenge to advance probabilistic forecasting for dengue epidemics. Proceedings of the National Academy of Sciences, 116(48), 24268-24274. https://doi.org/10.1073/pnas.1909865116

Lauer, S. A., Grantz, K. H., Bi, Q., Jones, F. K., Zheng, Q., Meredith, H. R., Azman, A. S., Reich, N. G., \& Lessler, J. (2020). The incubation period of coronavirus disease 2019 (COVID-19) from publicly reported confirmed cases: Estimation and application. Annals of Internal Medicine, 172(9), 577-582.

McAndrew, T. C., \& Reich, N. G. (2020). An expert judgment model to predict early stages of the COVID-19 outbreak in the United States. medRxiv, 2020.09.21.20196725. https://doi.org/10.1101/2020.09.21.20196 725

McGowan, C. J., Biggerstaff, M., Johansson, M., Apfeldorf, K. M., Ben-Nun, M., Brooks, L., Convertino, M., Erraguntla, M., Farrow, D. C., Freeze, J., Ghosh, S., Hyun, S., Kandula, S., Lega, J., Liu, Y., Michaud, N., Morita, H., Niemi, J., Ramakrishnan, N., ... Reed, C. (2019). Collaborative efforts to forecast seasonal influenza in the United States, 2015-2016. Scientific Reports, 9(1, 1), 683. https: //doi.org/10.1038/s41598-018-36361-9

Metaculus. (2020, June 22). A Preliminary Look at Metaculus and Expert Forecasts. https://www.metaculus. com/news/2020/06/02/LRT/

Our World in Data. (2020). COVID-19 Data Explorer. Our World in Data. https://ourworldindata.org/cor onavirus-data-explorer

PredictIt. (2021). PredictIt. https://www.predictit.org/

Recchia, G., Freeman, A. L. J., \& Spiegelhalter, D. (2021). How well did experts and laypeople forecast the size of the COVID-19 pandemic? PLOS ONE, 16(5), e0250935. https://doi.org/10.1371/journal.pone.0250935

Reich, N. G., Brooks, L. C., Fox, S. J., Kandula, S., McGowan, C. J., Moore, E., Osthus, D., Ray, E. L., Tushar, A., Yamana, T. K., Biggerstaff, M., Johansson, M. A., Rosenfeld, R., \& Shaman, J. (2019). A collaborative multiyear, multimodel assessment of seasonal influenza forecasting in the United States. Proceedings of the National Academy of Sciences, 116(8), 3146-3154. https://doi.org/10.1073/pnas.1812594116

Reich, N. G., McGowan, C. J., Yamana, T. K., Tushar, A., Ray, E. L., Osthus, D., Kandula, S., Brooks, L. C., Crawford-Crudell, W., Gibson, G. C., Moore, E., Silva, R., Biggerstaff, M., Johansson, M. A., Rosenfeld, R., \& Shaman, J. (2019). Accuracy of real-time multi-model ensemble forecasts for seasonal influenza in the U.S. PLOS Computational Biology, 15(11), e1007486. https://doi.org/10.1371/journal.pcbi.1007486

ReplicationMarkets. (2020). Replication Markets - Reliable research replicates... you can bet on it. https: //www.replicationmarkets.com/

Riutort-Mayol, G., Bürkner, P.-C., Andersen, M. R., Solin, A., \& Vehtari, A. (2020). Practical hilbert space approximate bayesian gaussian processes for probabilistic programming. https://arxiv.org/abs/2004.11408

RKI. (2021). RKI - Coronavirus SARS-CoV-2 - Aktueller Lage-/Situationsbericht des RKI zu COVID-19. https://www.rki.de/DE/Content/InfAZ/N/Neuartiges_Coronavirus/Situationsberichte/Gesamt.html

Scott, J. A., Gandy, A., Mishra, S., Unwin, J., Flaxman, S., \& Bhatt, S. (2020). Epidemia: Modeling of epidemics using hierarchical bayesian models. https://imperialcollegelondon.github.io/epidemia/

Servan-Schreiber, E., Wolfers, J., Pennock, D. M., \& Galebach, B. (2004). Prediction Markets: Does Money Matter? Electronic Markets, 14 (3), 243-251. https://doi.org/10.1080/1019678042000245254

Shaman, J., \& Karspeck, A. (2012). Forecasting seasonal outbreaks of influenza. Proceedings of the National Academy of Sciences, 109 (50), 20425-20430. https://doi.org/10.1073/pnas.1208772109

Sherratt, K., Abbott, S., Meakin, S. R., Hellewell, J., Munday, J. D., Bosse, N., Group, C. C. working, Jit, M., \& Funk, S. (2021). Exploring surveillance data biases when estimating the reproduction number: With insights into subpopulation transmission of Covid-19 in England. 2020.10.18.20214585. https: //doi.org/10.1101/2020.10.18.20214585

Stan Development Team. (2020). RStan: The r interface to stan. http://mc-stan.org/

Tetlock, P. E., Mellers, B. A., Rohrbaugh, N., \& Chen, E. (2014). Forecasting Tournaments: Tools for Increasing Transparency and Improving the Quality of Debate. Current Directions in Psychological Science, 23(4), 290-295. https://doi.org/10.1177/0963721414534257

Viboud, C., Sun, K., Gaffey, R., Ajelli, M., Fumanelli, L., Merler, S., Zhang, Q., Chowell, G., Simonsen, L., \& Vespignani, A. (2018). The RAPIDD ebola forecasting challenge: Synthesis and lessons learnt. Epidemics, 22, 13-21. https://doi.org/10.1016/j.epidem.2017.08.002

Xu, B., Gutierrez, B., Hill, S., Scarpino, S., Loskill, A., Wu, J., Sewalk, K., Mekaru, S., Zarebski, A., Pybus, O., Pigott, D., \& Kraemer, M. (2020). Epidemiological data from the nCoV-2019 outbreak: Early 
medRxiv preprint doi: https://doi.org/10.1101/2021.12.01.21266598; this version posted December 5, 2021. The copyright holder for this preprint (which was not certified by peer review) is the author/funder, who has granted medRxiv a license to display the preprint in perpetuity.

It is made available under a CC-BY 4.0 International license.

descriptions from publicly available data. http://virological.org/t/epidemiological-data-from-the-ncov2019-outbreak-early-descriptions-from-publicly-available-data/337

Yamana, T. K., Kandula, S., \& Shaman, J. (2016). Superensemble forecasts of dengue outbreaks. Journal of The Royal Society Interface, 13(123), 20160410. https://doi.org/10.1098/rsif.2016.0410 